\title{
Kinematic Basis for Body Specific Locomotor Mechanics and Perturbation Responses
}

\author{
Ryan B. Ross
}

Follow this and additional works at: https://researchrepository.wvu.edu/etd

\section{Recommended Citation}

Ross, Ryan B., "Kinematic Basis for Body Specific Locomotor Mechanics and Perturbation Responses" (2015). Graduate Theses, Dissertations, and Problem Reports. 6531.

https://researchrepository.wvu.edu/etd/6531

This Thesis is protected by copyright and/or related rights. It has been brought to you by the The Research Repository @ WVU with permission from the rights-holder(s). You are free to use this Thesis in any way that is permitted by the copyright and related rights legislation that applies to your use. For other uses you must obtain permission from the rights-holder(s) directly, unless additional rights are indicated by a Creative Commons license in the record and/ or on the work itself. This Thesis has been accepted for inclusion in WVU Graduate Theses, Dissertations, and Problem Reports collection by an authorized administrator of The Research Repository @ WVU. For more information, please contact researchrepository@mail.wvu.edu. 


\title{
Kinematic Basis for Body Specific Locomotor Mechanics and Perturbation Responses
}

\author{
Ryan B. Ross \\ Thesis submitted \\ to the Eberly College of Arts and Sciences \\ at West Virginia University \\ in partial fulfillment of the requirements for the degree of \\ Master of Science in \\ Biology \\ Kevin C. Daly, Ph.D., Chair \\ Andrew Dacks, Ph.D. \\ Sarah Farris, Ph.D.
}

Key Words: Locomotion, Neuromechanics, Biomechanics, Brachyuran, Decapod, Perturbation Copyright 2015 Ryan Ross 


\section{Kinematic Basis for Body Specific Locomotor Mechanics and Perturbation Responses}

Animals have evolved mechanical and neural strategies for locomotion in almost every environment, overcoming the complexities of their habitats using specializations in body structure and animal behavior. These specializations are created by neural networks responsible for generating and altering muscle activation. Species specific musculoskeletal anatomy and physiology determine how locomotion is controlled through the transformation of motor patterns into body movements. Furthermore, when these species specific locomotor systems encounter perturbations during running and walking their behavioral and mechanical attributes determine how stability is established during and after the perturbation. It is still not understood how species specific structural and behavioral variables contribute to locomotion in non-uniform environments. To understand how these locomotor properties produce unique gaits and stability strategies we compared three species of brachyuran crabs during normal and perturbed running. Although all crabs ran sideways, morphological and kinematic differences explained how each species produced its unique gait and stability response. Despite the differences in running behavior and perturbation response, animals tended to use locomotor resources that were in abundance during stabilizing responses. Each crab regained stability during the perturbation response by altering leg joint movements or harnessing the body's momentum. These species body designs and running behavior show how slight changes in body structure and joint kinematics can produce locomotor systems with unique mechanical profiles and abilities. Understanding how evolutionary pressures have optimized animals' locomotor ability to successfully move in different environments will provide a deeper understanding of how to mimic these movements through mathematical models and robotics. 


\section{Table of Contents}

Abstract Error! Bookmark not defined.

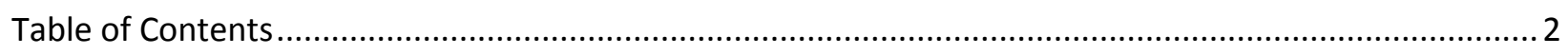

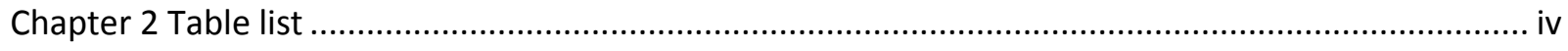

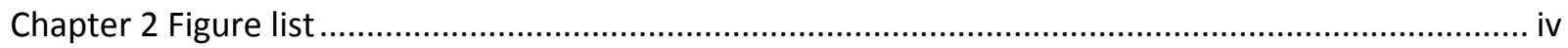

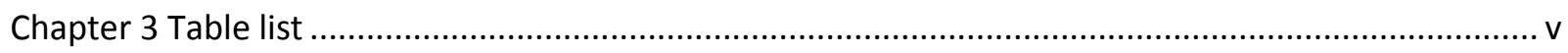

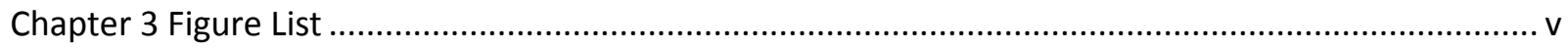

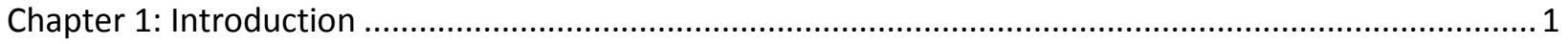

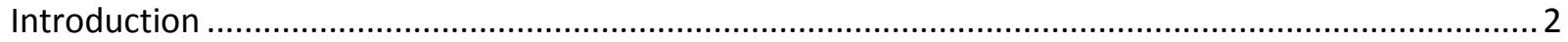

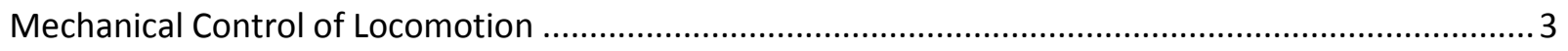

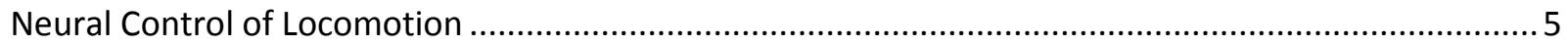

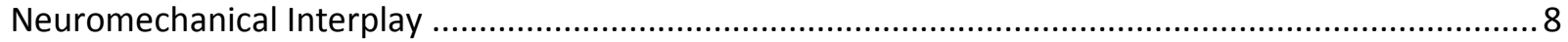

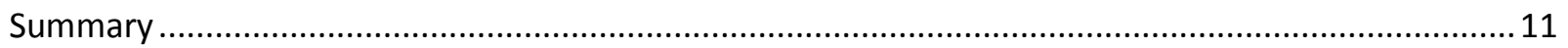

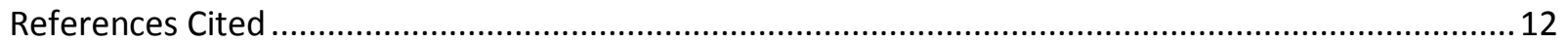

Chapter 2: Animal Structure and Joint Behavior determine Body Dynamics during Sideways Running in

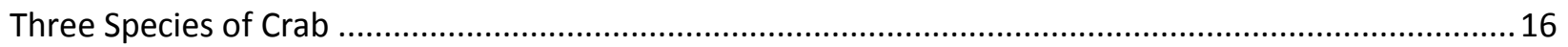

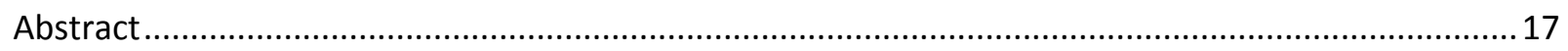

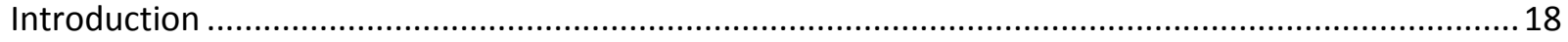

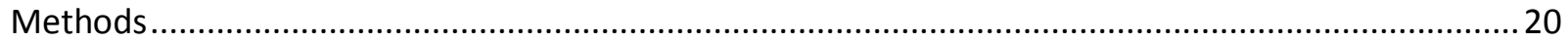

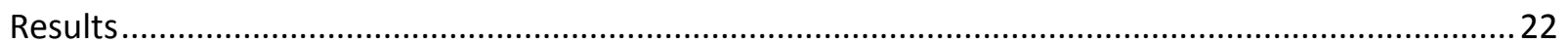

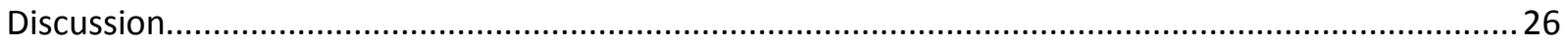

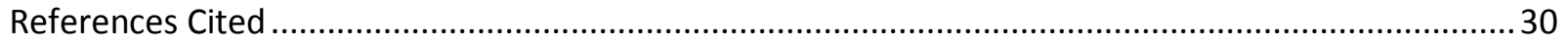

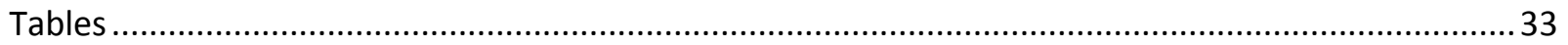

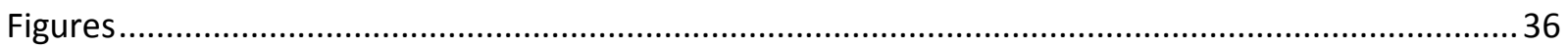

Chapter 3: Locomotor Control Strategy Determines Stability during Perturbation Recovery in Three

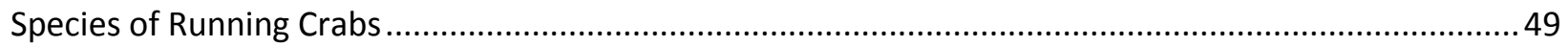

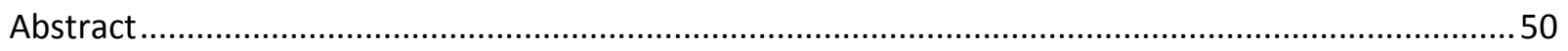

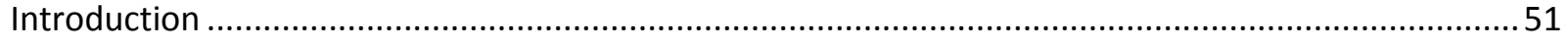

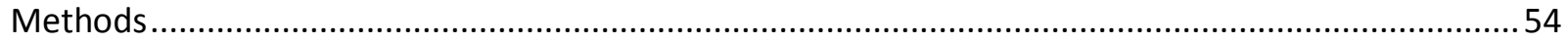

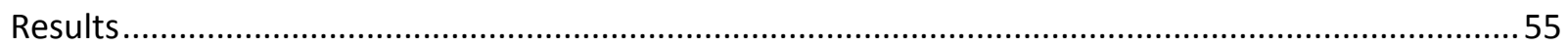




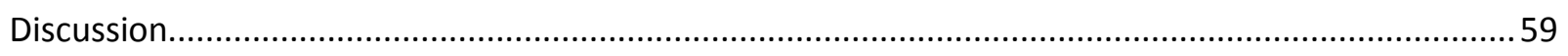

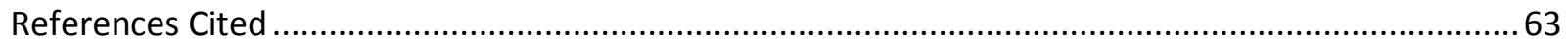

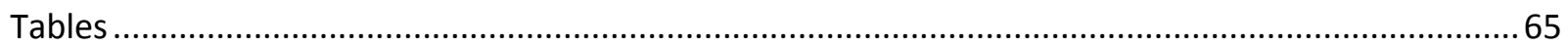

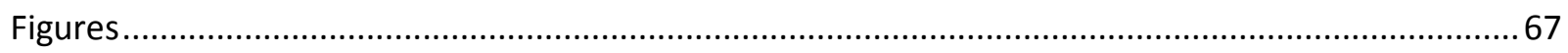

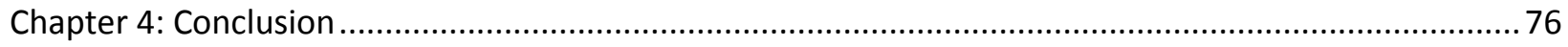

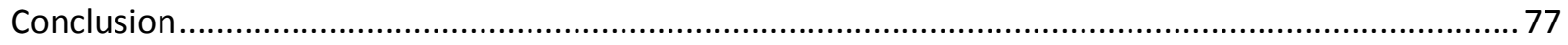

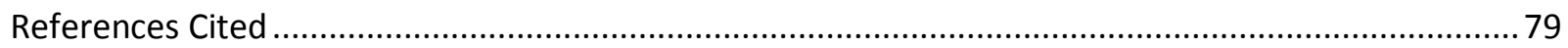

\section{Chapter 2 Table list}

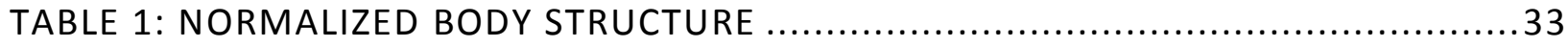

TABLE 2: NORMALIZED STANCE INITIATION .......................................... 33

TABLE 3: NORMALIZED CENTER OF MASS MOVEMENT .................................... 34

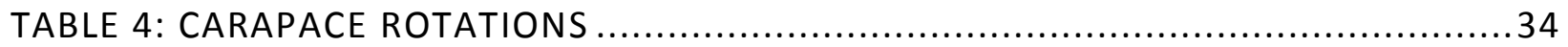

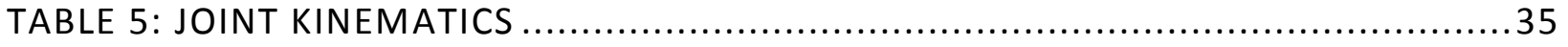

\section{Chapter 2 Figure list}

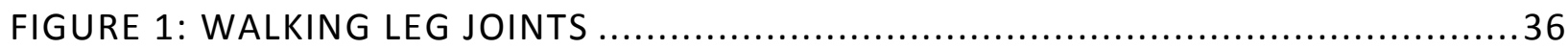

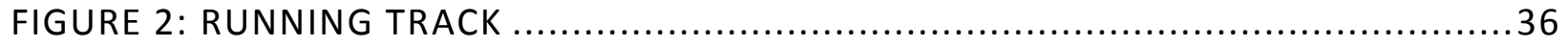

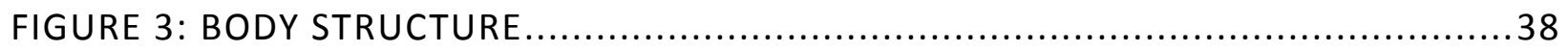

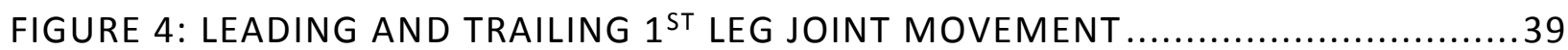

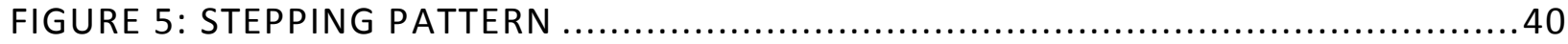

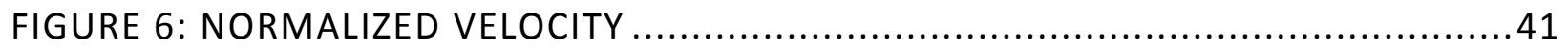

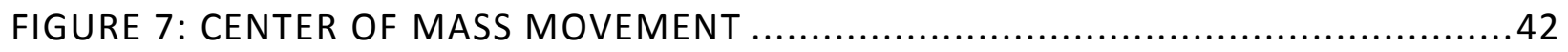

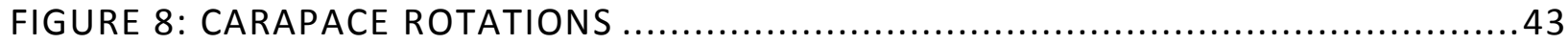

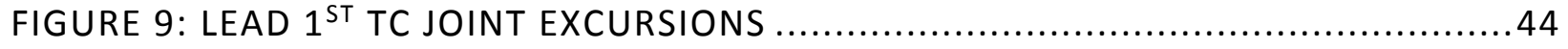

FIGURE 10: LEAD $3^{\text {RD }}$ CB JOINT EXCURSION AND CONTACT LIFT .......................45

FIGURE 11: TRAILING $2^{\text {ND }}$ AND $3^{R D}$ TC JOINT EXCURSIONS ..............................46

FIGURE 12: TRAILING $3^{\text {RD }}$ TC JOINT CONTACT-LIFT ...................................47

FIGURE 13: TRAILING $3^{\text {RD }}$ PD JOINT CONTACT ....................................... 48 


\section{Chapter 3 Table list}

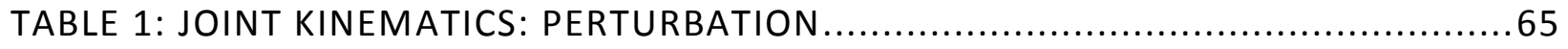

TABLE 2: MECHANICAL ENERGY RECOVERY ..........................................65

TABLE 3: KINETIC: POTENTIAL ENERGY RATIO ..........................................66

TABLE 4: PROBABILITY OF $4^{\text {TH }}$ WALKING LEG STEP DURING STRIDE ....................66

\section{Chapter 3 Figure List}

FIGURE 1: GHOST CRAB MECHANICAL ENERGY RETURN ..............................67

FIGURE 2: GHOST CRAB PERTURBATION RESPONSE: VELOCITY ..........................68

FIGURE 3: HALLOWEEN MECHANICAL ENERGY RECOVERY .............................69

FIGURE 4: HALLOWEEN CRAB LEAD $1^{\text {ST }}$ CB JOINT PERTURBATION RESPONSE ...........70

FIGURE 5: HALLOWEEN CRAB TRAILING $3^{\text {RD }}$ TC JOINT PERTURBATION RESPONSE .....71

FIGURE 6: HALLOWEEN TRAILING $3^{\text {RD }}$ PD JOINT PERTURBATION RESPONSE ............72

FIGURE 7: FIDDLER CRAB MECHANICAL ENERGY RECOVERY ..........................73

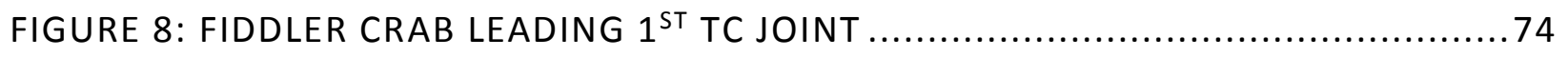

FIGURE 9: FIDDLER CRAB LEADING $3^{\text {RD }}$ AND $4^{\text {TH }}$ MC JOINT CONTACT ...................75 
Chapter 1: Introduction 


\section{Introduction}

Animals have evolved strategies for locomotion in almost every environment, overcoming the complexities of their habitat to reproduce, find food and avoid predators. All of these locomotor systems are controlled by neural networks, which are responsible for generating and altering locomotor behaviors through differential patterns of muscle activation. Although the nervous system is responsible for the initiation and maintenance of locomotion, it only plays a partial roll in the control of locomotion (Sponberg and Full, 2008). In particular, the time delays associated with sensory-motor feedback and muscle contraction times, limit the usefulness of sensory information available during very fast movements. These delays are too long to allow for the speed of compensatory action known to occur during rapid movement (Jindrich and Full, 2001). Thus there must be mechanical properties intrinsic to animal design that enables movement without detailed neural control. The result is a neuromechanical control system that uses motor output, sensory-motor feedback and mechanical interactions to control locomotion (Brown and Loeb, 1999).

Neuromechanical systems utilize the size, shape, and structural compliance of the animal and even the physical properties of the environment to determine how locomotion is controlled. When animals encounter perturbations during steady movement, the mechanical properties of limbs and muscles allow for immediate, passive stabilizing responses prior to sensory-motor feedback. These mechanical responses are probably maintained as neural feedback actively modulates motor output throughout subsequent step cycles. This interplay between the neural and mechanical responses allows the organism to maintain stable locomotion, while responding to changes in complex environments with very little time delay. Although multiple studies have been conducted on running in complex environments, none have directly compared how size, structural and behavioral differences among species affect normal movement and responses to unexpected perturbations.

Musculoskeletal and sensory-motor systems create stable locomotion in complex environments, using functional and anatomical principles to achieve reliable locomotion. To appreciate how musculoskeletal and neural control systems function during locomotion, it is beneficial to understand how the component parts operate independently and interdependently. The following sections will provide background on the physiology of musculoskeletal and neural systems important during animal locomotion. 


\section{Mechanical Control of Locomotion}

\section{Musculoskeletal system}

Traditionally the view of motor control was a hierarchy of commands descending through the nervous system directly controlling forces exerted on the environment. This view has dissipated with the discovery of pattern generating networks, intrinsic viscoelastic and contractile properties of muscles and the true nature of neural commands descending to sensorimotor systems (Gerritsen, 1998; Grillner, 1972; Loeb, 1995;). Some now view the neural control over locomotion more as a set of suggested actions that are translated into movement based on the current state of the musculoskeletal system and environment (Dickinson et al. , 2000). Inevitable time delays and computational limitations are assumed with neural control and sensory feedback. These delays limit the nervous system's ability to control every aspect of locomotion, with a multitude of joint angles and torques to consider which are constantly altered during movement (Brown and Loeb, 1999). This leaves the musculoskeletal system to function as a buffer during rapid locomotion, utilizing the intrinsic properties of body structures to offload some of the demands on the nervous system during creation of a stable gait in complex environments.

The intrinsic properties of muscles, tendons, and the skeletal system allow for passive 'preflexes' to counteract perturbation by returning the system to an equilibrium trajectory. Preflexes are in part, produced by muscle fibers, which are able to modulate contraction without direct neural input depending on current internal and external factors (Grillner, 1972). This modulation of contraction is exploiting the basic force-length and force-velocity relationships present in active muscles, which are directly affected by the joint angle and muscle geometry of the locomotor appendages (Wagner and Blickhan 1999; Cormie et al 2011). A seemingly ubiquitous feature of active muscle is that with increased fiber lengthening, the time course of contraction and relaxation is increased. An increase in contraction and relaxation time is thought to be created by an increase in $\mathrm{Ca}^{2+}$ affinity of myofilaments as muscle length is increased (Stephenson and Wendt, 1984). This feature allows muscles to compensate for the lack of available force at increased lengths by increasing contraction time, allowing for maximal muscle strain to be produced at a slower rate, possibly compensating for the negative effect of increased length on force production (Josephson 1999). The force-length relationship is present when active muscle is stretched, producing a mechanical response that is unique to the current state of the contractile and elastic components (Gerritsen et al 1998; Grillner, 1972). The mechanical features of muscles, as well as other intrinsic properties such as the series elasticity and force dependent deactivation, allow muscles to alter their 
function without direct neural input. Thus, the response of a locomotor system to a given perturbation depends on the current mechanical conditions created by the geometrical configuration of joints and the stiffness of muscles and connective tissue as well as neural feedback (Brown and Loeb, 1999).

\section{Muscle Contraction Mechanics}

Force of contraction, velocity of shortening and load determine the mechanical work available to muscles. Muscle force is influenced by many factors of contraction including the length-tension relationship, the force-velocity relationship and the degree of muscle activation, which determines the magnitude and time course of muscle activity over the contraction phase (Josephson 1999). These factors are ultimately products of muscle structure and are directly influenced by the length (total number of sarcomeres in series) and cross sectional area (CSA) of muscle.

The force-length relationship correlates muscle length with its ability to generate tension due to overlap of thick and thin filaments (Josephson, 1999). The force-length relationship explains that a muscle stretched beyond optimum length has decreased overlap of thick and thin filaments, resulting in a suboptimal number of cross bridge linkages available. Also the relationship between force and length explains that a muscle contracted beyond optimal length will decrease force production due to excess overlapping of thin filaments and compressive forces generated when the thick filaments push against Z-disk (Josephson 1999). This relationship in muscle contributes to the unique tension and force produced given the current geometric configuration of joints and allows for interpretation of neural signals to be dependent on the current limb and body position. Another innate property of muscle is the force-velocity relationship, which dictates that during contraction there is an inverse relationship between the force on a muscle and the velocity with which it can shorten (Josephson 1999). The force-velocity relationship dictates that two muscles with the same length, but different CSA will have distinctive abilities to generate force over the length of the contractile range. The muscle with the larger CSA will have the ability to generate greater absolute force although the optimal length and maximal velocity of both muscles is the same. Given both muscles maximum velocity is equal, the muscle with the greater CSA will be able to produce higher amounts of force at any velocity (Narici and Maganaris 2006).

These innate properties of muscles produce forces based on the neural input and current position of the muscle during contraction or relaxation. Another aspect of muscle function that is not altered by neural input is the series elastic component (SEC), which is created by the connective tissue and elastic 
components found within muscles and tendons (apodemes in arthropods). The SEC functions as a buffer during abrupt contractions and it has the ability to store potential energy as the tension in muscle is increased either by shortening or external forces (Hill 1950). The force produced during tension increase is transferred along the compliant components of the SEC that run the length of the muscle-tendon unit. The myofilaments, Z-disks, and tendons or apodemes of skeletal muscle form the SEC, and permit shortening of the contractile component (CC) without shortening of the entire muscle-tendon unit during isometric contraction (Josephson 1999).

The SEC permits the muscle-tendon unit to maintain lengths and velocities, which are greater than or less than that of the $\mathrm{CC}$, depending on whether the force is decreasing and the SEC is shortening or the force is increasing and the SEC is lengthening (Josephson 1999). In most movements limb inertia or external forces oppose contraction and enable mechanical energy to be stored in the SEC for subsequent release, enabling the production of a greater velocity than the CC could produce alone (Hill 1950). The ability to store and utilize mechanical energy stored in the SEC is necessary for movements such as running, jumping, and throwing. The stored potential energy enables muscles to perform oscillatory movements more efficiently, using elastic structures to recover energy lost by the body during prior movements (Biewener 1997).

Given the contractile mechanics of muscle and the variability in function as muscle state changes, it becomes evident that muscle function plays an important role in how locomotion is controlled. Understanding how muscles interpret neural signals becomes vital to understanding how movement is performed and implies that the structure and mechanical properties of animal muscle dictate how movement is performed, which may become even more important during rapid locomotion in complex environments. Collectively the size, shape and elasticity as well as recent and current activity of muscles make such an overall contribution to locomotory behaviors; it has been argued that the nervous system is only able to suggest movements to the musculoskeletal system, with muscles interpreting these suggestions based on current position (Loeb, 1995).

\section{Neural Control of Locomotion}

\section{Motor Neuron Function}

Motor neurons transmit muscle commands from the nervous system to individual muscle fibers. The synaptic connections between the nervous system and muscle are entirely excitatory in vertebrates, 
utilizing a single neurotransmitter to relay excitation to multiple motor units per muscle (Arrowsmith, 2007). A single motor neuron synapses with multiple muscle fibers, collectively known as a motor unit in vertebrates. In contrast, arthropods have relatively few motor neurons each with multiple synaptic connections to the same fiber. Arthropods are able to transmit excitatory and inhibitory signals along motor neurons, displaying multiple synaptic connections for each muscle fiber (Lang and Atwood, 1973). Both vertebrate and arthropod motor neurons form synaptic connections with muscle at the neuromuscular junction (NMJ) (Sieck and Prakash 1997).

Vertebrates produce graded muscle force through motor unit recruitment, generating different levels of muscle excitation based on the relative proportion of motor units activated for a muscle. Given the number of motor units present in vertebrate muscles, gradation of contraction can be produced by multiple combinations of recruitment and frequency modulation to alter active motor units (Broman et al 1985). The degrees-of-freedom created by the multitude of joints implies that there is likely to be some simplifying strategies to produce the correct amount of muscle tension over an appropriate time period without burdening the central nervous system with the complexity (Hodson-Tole 2008). One simplifying strategy supported by research has been the 'size principle', stating that an orderly recruitment of motor units begins with the smallest and proceeds to maximal tension by recruiting subsequently larger units (Wakeling 2005). This simplified size principle also corresponds to derecruitment of motor units, which allows for gradation of muscle relaxation as larger units are derecruited first followed by successively smaller motor units (Hudson-Tole 2008). The size principle has also been shown to correspond with motor unit inhibition, with motor neuron inhibition allowing for smoother generation of contraction and relaxation as successively larger motor units become activated (Broman et al., 1984).

In contrast, the arthropod motor control systems utilize very few motor neurons to coordinate motor control, relying heavily on frequency modulation and the matching of excitatory and inhibitory signals (Atwood, 1963). A single muscle fiber can be innervated by multiple connections along its length, synapsing with just one type of motor neuron or a combination of phasic, tonic and inhibitory motor neurons. This multi-terminal innervation allows for synchronous contraction utilizing multiple graded responses along the length of the fiber (Willmer et al, 2005). Inhibitory and excitatory motor neurons provide arthropods with a mechanism for not only muscle excitation, but also for inhibition of muscle activity through polyterminal innervation. This allows the motor control system to have another level of flexibility in arthropods, controlling not only contraction but also the inhibition of contraction. Excitatory 
and inhibitory motor axons usually innervate muscles in parallel (similar branching patterns), and tend to make synaptic connections with muscle fibers in very close proximity, thus arthropod muscles are multineuronal (Atwood and Bittner, 1970). Many muscle fibers in arthropods are innervated by two or three excitatory neurons (one of which is usually fast) and one or two inhibitory neurons (Willmer et al 2005).

Although there are morphological and physiological differences between vertebrate and arthropod motor neurons, their function is similar in that both must convey signals from the nervous system to muscles. Although vertebrates and arthropods evolved legged locomotion independently (Ritzmann et al, 2004), both have developed control strategies dependent on the functional and anatomical properties of motor neurons. Vertebrates rely on a large numbers of motor neurons recruited in an orderly fashion to produce the appropriate amount of activity. Arthropods depend on excitatory and inhibitory signals, using frequency and magnitude of signals to produce the appropriate amount of activity in muscles. Arthropods and vertebrates have developed contrasting systems for muscle activation, solving the same problem with different solutions (Belanger 2005). Muscle activation strategies built into the functional and anatomical properties of motor neurons make the control of muscle activity easier for the nervous system on a single muscle level and when combined with the synergistic activity of muscle groups, can simplify the control of entire limbs (D'Avella et al, 2004). Given that strategies for motor output have evolved to enable control of individual muscles, groups of muscles and synergistic muscles; full body control strategies may have evolved across the sensory-motor and musculoskeletal systems. These full body control strategies would utilize the size, shape, muscle mechanics and behavior of the animal to produce stable locomotion and simplify the control of activities. Therefore, a productive approach to understanding these full body control strategies will be to determine how mechanical and behavioral properties affect motor output during running in uniform and complex environments.

\section{Mechanosensory Feedback}

Mechanosensory inputs are involved in many features of adaptive motor behavior and are able to initiate or terminate motor output as well as modulate movement amplitude or duration (Cattaert 2002). Sense organs located in the limbs play an important role in the control of locomotion, providing the nervous system with information concerning the state of the animal's joints and body position. Mechanosensory organs serve to monitor actions and positions of the body to ensure that: movement is sufficient, and successful adjustments to posture and locomotion are made during environmental variations, and perturbations are detected and followed by the appropriate compensatory adjustments 
(Zill et al 2004). Although sensory information is vital to the maintenance of stable locomotion, it is not necessary for the generation of rhythmic motor patterns. Rhythmic limb moevements are initiated by the CNS and have been shown to be present (though clearly altered) without sensory input in arthropods and vertebrates (MacKay-Lyons 2002; Wilson 1961).

The context in which sensory information is perceived is an important factor driving its control over locomotion. In the shore crab and crayfish, the action of sensory reflexes is quite different in active versus quiescent behaviors. Passive movements about a proximal joint generate resistance reflex whereas active movements with similar characteristics produce no reflex (DiCaprio and Clarac, 1980; Ray et al 1997). This implies that the reflex during quiescence behavior is homeostatic, utilizing negative feedback, whereas during active locomotion positive feedback prevails. These positive feedback reflexes are thought to provide load-compensating effects and possibly provide a mechanism for entrainment of locomotor rhythms (Skorupski and Sillar, 1986). During rapid movement, these positive feedback reflexes would allow for active responses following a perturbation, reinforcing the musculoskeletal preflexes already present.

\section{Neuromechanical Interplay}

The interplay between neural information and the mechanical behavior of animals may offload some of the requirements of the nervous system during locomotion (Belanger and Trimmer, 2000: Sponberg and Full, 2008; Jindrich and Full, 2002). This neuromechanical interplay is able to utilize shape,

size, and structural impedance of an animal as well as the physical properties of the environment to determine how motor control is conducted. Thus, a true understanding of this neuromechanical system and the musculoskeletal system will come from stable movement during steady conditions as well as dynamically stable locomotion through complex terrain (Nishikawa et al, 2007).

While sensory feedback and reflexes seem to dominate motor control during slow statically stable movements, feedforward musculoskeletal preflexes become more prevalent as speed increases. This feedforward musculoskeletal activity stabilizes locomotion mechanically without neural input. This contrast creates two independent forms of stability in animals, static and dynamic (Karcik et al, 2003). Static stability is prevalent in sprawled posture, slow moving animals that are able to maintain a base of support under the center of mass (COM) throughout the gait. As speeds increase the criteria for statically stable gaits is degraded as the COM travels closer to the edge of its support base (Full et al, 2002). Many legged terrestrial locomotors meet the statically stable criteria over a wide range of speeds, but when 
speed is increased static stability is reduced (Ting et al, 1994). Dynamic stability is used to bridge periods of static instability, utilizing body momentum and the mechanical properties of the limbs at high velocities (Jindrich and Full 2002). Periods of static instability are particularly prevalent in animals that utilize fewer legs and/or aerial phases during locomotion causing these animals to depend on dynamic stability throughout the gait (Karcnik, 2004).

Dynamic stability can be characterized by steady state variables that change or fluctuate in a characteristic fashion. In this way, dynamic stability can be described by state variables such as velocities, joint angles, step cycle and COM position (Full et al, 2002). These state variables change over time in stable locomotion as a consequence of the system dynamics, oscillating in a rhythmic pattern. Time independent rhythmic patterns of state variables can be compared, allowing for the behavior of variables to be observed relative to one another, no longer considering time. These comparisons can lead to the formation of duty cycles capable of depicting the change in all state variables over one step cycle, defining an equilibrium state or trajectory. In this way state variables allow for quantification of a dynamic systems return to equilibrium following a disturbance, defined by the time and pattern of variable change (Full et al, 2002).

The challenges of dynamic stability quantification can be avoided by analysis of simple models that attempt to capture some aspect of state variables. The simplest of these models attempts to estimate the movements of the COM around a few step cycles. These models do not attempt to recreate the multidimensionality of animals, but simply utilize knowledge of actual animals and attempt to explain simple mechanisms by which motor function may be controlled (Full and Koditschek 1999). Many of these models appear to capture the compliant structures discussed above, necessitating the application of spring like structures in almost every successful model. Although these models reduce the complexity of animals away from reality, they can be a useful tool in capturing how a system that utilizes neural input to control the mechanics of muscles is able to produce stable movement.

\section{Simple Models for Locomotion: Templates of Movement}

Models capable of simulating simple locomotion give a prediction of the movement of the COM of an animal. These reductive models or templates parsimoniously encode the movement of an animal, revealing the basic principles of motion without considering the finer details of body structure (Full and Koditschek, 1999). The most prominent and widely used of these models in locomotion is the spring-like inverted pendulum (SLIP) model for COM movement. This SLIP model is composed of a pendulum and a 
spring in series, which are utilized in combination or individually. The SLIP model proposes a mechanism for minimizing energy expenditure through storing and recovering the energy during each phase of the step cycle. It explains two mechanisms for alternatively storing and recovering energy; first the pendulum effect of exchange between gravitational potential energy and kinetic energy and second the spring like effect due to exchanges of mechanical energy stored in muscles and tendons which is recovered as kinetic and gravitational energy (Cavagna et al, 1977).

Application of the SLIP model to actual data from animal locomotion is possible for a wide range of locomotor designs given a few experimental parameters, model assumptions and behavioral exclusions. The SLIP model can be fit to gaits whose dynamics are described by alternating propulsive phases with a bouncing COM at a constant velocity (Blickhan 1989; Blickhan and Full, 1993). Multiple methods and many types of data can be applied to the SLIP model, which allow for estimations of leg stiffness and energy transfer during animal locomotion. Blickhan and Full, (1993) compared multiple animal designs over a wide range of gaits, utilizing total force measurements to determine the angle of attack and the springs stiffness (Blickhan, 1989). Another group of investigators sought to fit real animal data to the SLIP model, ignoring total force measurements while applying kinematic data. Srinivasan and Holmes (2007) were able to infer leg stiffness from measurements on a running animals, using only speed, step length and duty factor (fraction of full stride for which either leg is in ground contact). They attempted to fit the measurements easily obtained from animals (e.g. leg length and mass) to the model, attempting to infer the stiffness of the virtual leg. The stiffness of the leg is thought to be largely a behavioral choice, depending on the activation of antagonistic muscles and the preferred gait (increase in leg stiffness decreases duty factor in altering stance cycle; Srinivasan and Holmes, 2007).

Although the SLIP can qualitatively reproduce the COM movement of running behavior in many locomotion designs, it is unable to capture the dynamic stability associated with the compliant structures of the musculoskeletal system. (Srinivasan and Holmes, 2007). Even though the SLIP model is unable to capture the variables associated with dynamic stability, it has been shown to provide insight into the running behavior of crabs at varying speeds (Blickhan and Full, 1987). Utilizing this technique, we hope to understand how the mechanical energetics in different species of crab are affected by the size, body structure, speed and running behavior in complex environments. This model will also show how these mechanical effects determine their response to perturbations and provide insight into how they affect the efficiency of running. 


\section{Summary}

Locomotor control studies have historically focused on the function of animals during walking in uniform environments. Although these studies have improved our understanding of neural and mechanical motor control strategies, it is still unknown how locomotion is controlled at fast speeds in non-uniform environments. As speed and environmental complexity increase, it may be that the musculoskeletal system can compensate for deficiencies in neural control by using the elasticity of muscles and tendons to buffer environmental perturbations and the intrinsic properties of muscles to alter contraction and relaxation independent of neural control (Brown and Loeb, 1999). Although the nervous system is responsible for the initiation, maintenance and alteration of motor patterns, the mechanical properties of animals play an important role in the production and stabilization of locomotion, especially during rapid movement in natural environments.

It is still unknown how motor control strategies and body mechanics interact during animal locomotion to produce the unique gaits seen throughout the animal kingdom. The purpose of this research is to understand how structural and behavioral differences created by evolutionary pressures allow animals to move effectively in their complex habitats. Our results improve the understanding of how locomotion is controlled during stable movement under steady conditions as well as locomotion through complex terrain and during unexpected perturbations from loss of traction (Nishikawa et al, 2007). The following two chapters explain how species specific structural and behavior differences produce running ability and stability. Chapter 2 aims to characterize species specific locomotor patterns and how evolutionary pressures may have produced these structural and behavioral differences. Chapter 3 utilizes these species specific differences to explain responses to slippery surface perturbations and determine variables important for stability. Chapter 4 emphasizes the importance of the research for understanding evolutionary pressures associated with locomotion and how these optimized locomotor strategies can provide insight for biomimetics. 


\section{References Cited}

Arrowsmith, J.E. (2007) The Neuromuscular Junction. Journal of Surgery (Oxford). 25(1): 105-111

Atwood, H. (1967) Crustacean neuromuscular mechanisms. American Zoologist. 7(3): 527-552

Attwood, H. L. (1973) An attempt to account for the diversity of crustacean muscles. American Zoologist. 13(2): 357-378

Atwood, H.L. and G.D. Bittner (1970). Matching of Excitatory and Inhibitory Inputs to Crustacean Muscle Fibers. Journal of Neurophysiology. 34(1): 157-170

Belanger, J. (2005) Contrasting tactics in motor control by vertebrates and arthropods. Integrative and Comparative Biology 45 (1): 672-678

Belanger, J. and B. Trimmer (2000) Combined kinematic and elctromyographic analyses of proleg function during crawling by the caterpillar Manduca sexta. Journal of Comparative Physiology. A: 186: 1031-1039

Biewener, A. A. (1997) Effects of Elastic Energy Storage on Muscle Work and Efficiency. Journal of Applied Biomechanics, 13(4), 422-426

Blickhan, R. (1989) The Spring-Mass Model for Running and Hopping. Journal of Applied Biomechanics. 22: 1217-1227

Blickhan, R. and R. Full (1987) Locomotion energetics of the ghost crab II. Mechanics of the center of mass during walking and running. Journal of Experimental Biology. 130: 155-174

Blickhan, R. and R. Full. (1993) Similarity in multilegged locomotion: Bouncing like a monopode. Journal of Comparative Physiology A. 173: 509-517

Broman, H., Deluca, C.J., and B. Mambrito (1984). Motor Unit Recruitment and Firing Rates Interaction in the Control Of Human Muscles. Journal Brain Research. 337 (): 311-319

Brown, I. E., and G. E. Loeb (1999) A reductionist approach to creating and using neuromusculoskeletal models. In: Biomechanics and Neuro-Control of Posture and Movement, Ed. J. Winters and P. Crago. pp. 148-163. New York, Springer-Verlag

Burke, R.E. (2010) Comprehensive Physiology In: Handbook of Physiology. The Nervous System (ed. V.D. Brooks), pp. 345-422. American Physiology Society, Bethesda

Cattaert, D., Bon, M. L., and D. L. Ray. (2002) Efferent Control in Crustacean Mechanoreceptors. Microscopy Research and Technique. 58: 312-324 
Cavagna, G.A., Heglund, N.C., and R. Taylor. (1977) Mechanical work in terrestrial locomotion: two mechanisms for minimizing energy expenditure. American Journal of Physiology. 233: 243-261

Cormie, P., McGuigan, M. R., and R. U. Newton. (2011) Developing Maximal Neuromuscular Power. Journal of Sports Medicine 41(1): 17-36

D'Avella, A., Saltiel, P. and E. Bizzi (2003) Combinations of muscle synergies in the construction of a natural motor behavior. Nature Neuroscience. 6: 300-308

DiCaprio, R. A. and F. Clarac. (1980) Reversal of a Walking Leg Reflex Elicited by a Muscle Receptor. Journal of Experimental Biology. 90: 197-203

Dickinson, M., Farley, C., Full, R., Koehl, M., Kram, R. and S. Lehman (2000) How animals move: an integrative view. Science. 288: 100-106

Full, R. and D. Koditschek. (1999) Templates and Anchors: Neuromechanical Hypotheses of Legged Locomotion on Land. Journal of Experimental Biology 202: 3325-3332.

Full, R., Kubow, T., Schmitt, J., Holmes, P. and D. Koditschek (2002) Quantifying Dynamic Stability and Maneuverability in Legged Locomotion. Integrative and Comparative Biology. 42: $149-157$

Gerritsen K., Bogert, A., Hulliger, M., and R. Zernicke. (1998) Intrinsic Muscle Properties Facilitate Locomotor Control- A computer Simulation Study. Motor control. 2: 206-220

Grillner, S (1972) The Role of Muscle Stiffness in Meeting the Changing Postural and Locomotor Requirements for Force Development by the Ankle Extensors. Acta Physiologica Scandinavica 86: 92-108

Hill, A. V. (1950). The series elastic component of muscle. Royal society of London. Series B, Biological Sciences. 137(887): 273-80

Hodson-Tole, E.F. and J.M. Wakeling (2009) Motor unit recruitment for dynamic tasks: Current understanding and future directions. Journal comparative Physiology B. 179(1): 57-66

Holmes, P., Full, R., Koditschek, D., and J. Guckenheimer. (2006) The Dynamics of Legged Locomotion: Models, Analyses, and Challenges. Society for Industrial and Applied Mathematics. 48: 207-304

Jindrich, D. and R. Full (2002) Dynamic stabilization of rapid hexapedal Locomotion. Journal of Experimental Biology. 205: 2803-2823 
Josephson, Robert K. (1999). Dissecting Muscle Power Output. Journal of Experimental Biology. 202: 3369-75

Karcnik, T. (2004) Stability in legged locomotion. Biological Cybernetics. 90: 51-58

Lang, F. and H. L. Atwood. (1973). Crustacean Neuromuscular Mechanisms: Functional Morphology of Nerve terminals and the Mechanism of Facilitation. American Zoologist. 13(2) 337-355

Loeb, G. E. (1995). Control implications of musculoskeletal mechanics. In Engineering in Medicine and Biology Society, 1995., IEEE 17th Annual Conference (Vol. 2, pp. 1393-1394). IEEE.

Narici, M. and Maganaris, C. (2006). Chapter 9: Muscle Architecture and Adaptions to Functional Requirements. R. Bottinelli and C. Reggiani (Eds). Skeletal Muscle Plasticity in Health and Disease (265-88). New York, NY: Springer

MacKay-Lyons, M. (2002) Central Pattern Generation of Locomotion: A Review of the Evidence. Journal of American Physical Therapy Association. 82: 69-83

Nishikawa, K., Biewener, A. A., Aerts, P., Ahn, A. N., Chiel, J. H., Daley, M. A., Daniel, T. L., Full, R. J., Hale, M. E., Hedrick, T. L., Lappin A. K., Nichols, R., Quinn, R. D., Satterlie, R. A., and B. Szymik. (2007) Neuromechanics: an integrative approach for understanding motor control. Integrative and Comparative Biology. 47: 16-54

Ray, D. L., Clarac, F., and D. Cattaert. (1997) Functional Analysis of the Sensory Motor Pathway of Resistance Reflex in Crayfish. II. Integration of Sensory Inputs in Motor Neurons. Journal of Neurophysiology. 78: 3144-3153

Ritzmann, R., Quinn, R., and M. Fischer (2004) Convergent evolution and locomotion through complex terrain by insects, vertebrates and robots. Arthropod Structure and Development. 33: 361-379

Skorupski and Sillar (1986)

Sieck G. C. and Prakash, Y.S. (1997). Morphological Adaptations of Neuromuscular Junctions Depend on Fiber Type. Journal Applied Physiology. 22(3) 197-230

Sponberg, S. and R. J. Full. (2008) Neuromechanical response of musculo-skeletal structures in cockroaches during rapid running on rough terrain. Journal of Experimental Biology. 211: 433-446 
Srinivasan, M. and P. Holmes (2007) How well can spring-mass-like telescoping leg models fit multi-pedal sagittal-plane locomotion data? J. of Theoretical Biology. 255: 1-7

Stephenson, D., and I. Wendt (1984) Length dependence of changes in sarcoplasmic calcium concentration and myofibrillar calcium sensitivity in striated muscle fibers. Journal Muscle Research and Cell Motility. 5: 243-272

Ting, L. H., Blickhan, R., R. J. Full. (1994) Dynamic and static stability in hexapedal runners. Journal Experimental Biology. 197: 251-269

Wagner, H. and R. Blickhan (1999) Stabilizing Function of Skeletal Muscles: an Analytical Investigation. Journal theoretical Biology. 199: 163-179

Wakeling (2005) Motor unit recruitment during vertebrate locomotion. Animal Biology. 55, 4158

Willmer, P., Stone, G., and I. Johnston (2005). Chapter 9-Excitable Tissues: Nervous System and Muscles. In P. Willmer, G. Stone, and I. Johnston. Environmental Physiology of Animals: Second Edition. (pp.223-342). United Kingdom. Blackwell publishing.

Wilson, D. M. (1961) The Central Nervous Control of Flight in a Locust. Journal of Experimental Biology. 38: 471-490

Zill, S., Schmitz, J. and A. Buschges (2004) Load sensing and control of posture and locomotion. Arthropod Structure and Development. 33: 273-286 
Chapter 2: Animal Structure and Joint Behavior determine Body Dynamics during Sideways Running in Three Species of Crab 


\begin{abstract}
Animals have evolved locomotion strategies for most terrestrial environments, allowing them to overcome complex terrain and interact with their habitat. Species differences in anatomical structure have a profound effect on how locomotion is controlled, dictating how motor patterns are transformed into physical movement. It remains unclear how these species specific structural properties affect gait dynamics. Furthermore, it is unknown how evolutionary pressures mold the mechanical and behavioral characteristics of animals to allow for successful locomotion in different environments. For this reason we compared the kinematics of running in three brachyuran crab species to understand how structural and behavior differences dictate movement. Each species' distinct stepping patterns and joint mechanics created unique gait dynamics during running. Although only sideways running was examined, morphology and joint behavior explain the unique gaits of each species and resulting center of mass movements. These unique gait dynamics are a product of evolutionary pressures that shape the body structure and locomotor behavior, adaptively tuning each species to their specific behavioral ecology.
\end{abstract}




\section{Introduction}

Animals have adapted locomotor strategies for almost every environment, successfully overcoming variations in substrate, uneven surfaces, and unexpected perturbations. All of these locomotor strategies are mediated by neural networks, which are responsible for generating and altering locomotor patterns through muscle activation. Although neural tissue is responsible for the initiation and maintenance of locomotion, the nervous system only plays a part in the control of locomotion (Dickinson et al, 2000). The mechanical properties of musculoskeletal systems dictate how neural commands are translated into movement and are vital during the control of locomotion in complex environments. These mechanical properties are produced by limb structure and muscle function and integrate with neural processing to create a neuromechanical system that is able to produce stable movement (Nishikawa et al, 2007). Although it is known that the mechanical properties of animals play a significant role in locomotion, it is still unclear how subtle variations in body structure and locomotor preferences determine the mechanics of movement.

Neuromechanical systems exploit the size, shape, and structural compliance of the animal and even the physical properties of the environment to optimize locomotor control. Comparative studies of animals with distinct body structures have identified several important mechanical variables that shed light on the factors associated with different locomotor strategies (Full and Farley, 2000). These differences are beginning to explain the properties that determine how locomotion is controlled in complex environments and how different species' body structures have evolved to move effectively in their respective environments.

Viewing locomotion as a purely neurally controlled process where the musculoskeletal apparatus simply responds to the commands of the nervous system is a flawed and counterproductive approach to the study of locomotion (Zajac, 1989). The flow of information during locomotion is a closed loop system where joint and muscle sensors feedback information regarding the current position of the limbs and body position, velocity, and force which in a way makes the nervous system the initiator and planner of movement while the musculoskeletal system interprets mechanical cues to neural feedback and translates motor output into 
movement (Full and Farley, 2000). Furthermore, interplay within the musculoskeletal system, due to the mechanical properties of muscles, creates unique responses to neural signals in a state dependent manner (Biewener and Gillis, 1999). The musculoskeletal system also responds to perturbations independently using the structural compliance of the system and the animal's momentum (Jindrich and Full, 2002; Loeb, 1995; Chapter 3).

Although muscles provide the mechanism for force creation, they also produce the breaking forces that decrease kinematic energy and buffer movement in rhythmic systems (Azizi, 2014). The series elastic component (SEC) of muscle is composed of the elastic contractile and connective tissue found along the length of muscles. The SEC functions as a buffer during abrupt contractions and has the ability to store energy as the tension in muscle is increased, either by active shortening or external forces (Hill 1950). The ability to store and utilize mechanical energy held in the SEC is necessary for such movements as running, jumping, and throwing (Josephson, 1999). This stored elastic energy enables muscles to perform oscillatory movements more efficiently, using elastic structures to recover energy lost by the body during prior movements (Biewener 1997).

Analyzing closely related species with similar body structure and movement preferences can produce insights into how locomotor capability can be altered through the evolutionary pressures of the species' respective environments (Doran, 1993). Morphology can greatly affect the locomotor ability of animals and even slight changes in body structure can result in completely different modes of movement including body orientation (Vidal-Gadea et al, 2007), as well as gait characteristics and limb usage (Renous et al, 2008). Even closely related species with very similar body structures can display significantly different abilities in terms of speed and agility, as well as preferences in joint usage and perturbation recovery (Chapter 3).

Although a large amount is known about mechanics of locomotion, most studies have analyzed animals in isolation without comparing closely related species to understand how body structures dictate biomechanics during running. The goal of this research was to characterize the kinematics associated with fast sideways running in three species of brachyuran crabs, using limb morphology and body structure to explain the differences in locomotor ability. Using closely related species with similar movement preferences will allow for insights into how slight changes 
in morphology determine the locomotor ability of animals and will elucidate how evolution shapes structure and behavior to create different locomotor strategies. We therefore tested the following hypotheses: (i) Crab speed is dictated by their relative limb structure and preference in joint usage. (ii) Preferences in joint usage influence movement of the carapace and center of mass resulting in significantly different locomotor strategies.

\section{Methods}

\section{Experimental Subjects}

Three species of crustaceans were used in the present study: Uca pugilator (fiddler crab)(Carolina Biological Supplies), Ocypode quadrata (ghost crab)(Gulf Specimen Marine Laboratories, Inc), and Gecarcinus quadrates (Halloween crab)(Reptile City Pet Supply Co.). All crabs were maintained at $24{ }^{\circ} \mathrm{C}$ on a $12 \mathrm{hr}$ light cycle and fed three times a week. Fiddler crabs were fed crab pellets (Carolina Biological Supplies), ghost crabs were fed frozen freshwater smelt, and Halloween crabs were fed fresh spinach, apples, and Hermit Crab food (Carolina Biological Supplies). These species were selected because of differences in weight range, body proportions and ecological niches.

\section{Running Track}

We used a high friction, sandpaper substrate track painted white on the floor and walls to promote contrast. Three tracks were used for the different sizes of crabs; $50 \times 11 \times 19 \mathrm{~cm}$, $30 \times 7 \times 12 \mathrm{~cm}$ and $20 \times 7 \times 12 \mathrm{~cm}(\mathrm{X}-\mathrm{Y}-\mathrm{Z})$. Four cameras were placed in contralateral positions along the length of the $19 \times 50 \mathrm{~cm}$ track, with a camera at either end focused on the center of the track at $45^{\circ}$ angle and two cameras $15 \mathrm{~cm}$ from both ends of the track focused on the center at a $25^{\circ}$ angle (Fig. 1).

\section{Experimental protocols}

For each trial, crabs were placed at one end of the tunnel and induced to locomote with a running or trotting gait. To induce locomotion down the track in the desired direction, animals were released and immediately tapped on the trailing legs. In addition a fictive burrow was placed at the far end of the track (Walls and Layne, 2009). Each "run" was recorded with four cameras (Fuji Film model Finepix HS10 cameras, FujiFilm Corporation, Akasaka 9-Chrome, 
Minato-ku, Tokyo 107-0052, Japan). Prior to recording animals were marked with highcontrasting points on the carapace and at the distal ends of each segment of the walking legs, although the carpus-propus joint was ignored as it does not produce much movement during locomotion in these species. Each point was created by covering the area with Whiteout ${ }^{\circledR}$ and placing a dot of black nail polish in the location of interest to increase contrast. Each camera captured 120 frames s $\mathrm{s}^{-1}$ for Ghost and Fiddler crabs and 60 frames s $\mathrm{s}^{-1}$ for the Halloween crabs. The spatial resolution was $1.0 \mathrm{~mm}, 0.35 \mathrm{~mm}$ and $0.4 \mathrm{~mm}$ for the three running track sizes $50 \times 11 \times 19 \mathrm{~cm}, 30 \times 7 \times 12 \mathrm{~cm}$, and 20x7x12 cm (X-Y-Z-axis), respectively. The calibration object used to define the recording space covered $100 \%$ of the data collection area for each track, no movements were recorded outside the spatially defined recording area. All of the Ghost crabs and the Halloween crabs $>20 \mathrm{~g}$ were run on the 50x11x19 cm track, Halloween crabs $<20 \mathrm{~g}$ and Fiddler crabs $>3 \mathrm{~g}$ were run on the $30 \times 7 \times 12 \mathrm{~cm}$ track, and Fiddler crabs $<3 \mathrm{~g}$ were run on the $20 \times 7 \times 12 \mathrm{~cm}$ track. Recordings from all cameras were synchronized using a light emitting diode visible in the field of view of all cameras. Three floodlights surrounded the track and provided illumination during trials; lights were turned off during rest periods to avoid raising the track temperature. Video recordings were converted to .avi files and uploaded into Vicon Motus motion analysis software (Vicon Motus Motion Systems Inc.) for 3-dimensional analysis using the direct linear transform (Abdel-Aziz and Karara 1971).

\section{Motion Analysis}

\section{Body Dynamics and Center of Mass Movement}

Angular excursions of roll, pitch, and yaw were determined for the body of each species using points on the carapace placed at the rostrum and immediately above the L4/T4 TC joints on the ventral carapace. Roll was determined by comparing the lateral carapace vector which extended across the carapace from the points placed above the L4/T4 TC joints to the substrate plane to. Pitch was determined by comparing the ventral carapace vector which extended from the rostrum down the midline of the carapace to the substrate plane. Yaw was determined by comparing the lateral carapace vector to the vertical sidewall plane. Roll, pitch and yaw excursions were determined by the difference between the maximum and minimum values during a single stride. 
Change in normalized center of mass (COM) height $(\Delta \mathrm{H})$ was determined by the difference between the maximum and minimum height during each stride divided by the animal's carapace

width $\left.(\mathrm{CW})\left(\Delta \mathrm{H}=\mathrm{H}_{\max }-\mathrm{H}_{\min }\right) / \mathrm{CW}\right)$. Change in normalized velocity $(\Delta V)$ was the difference in maximum and minimum velocity during each stride divided by the animal's carapace width $\left(\left(\Delta \mathrm{V}=\mathrm{V}_{\max }-\mathrm{V}_{\min }\right) / \mathrm{CW}\right)$. Normalized average velocity was the average velocity of the running animal over the course of two strides divided by carapace width.

Leg Kinematics

Joint angles of the distal leg segments (merus, carpus, propus, dactyl) were measured directly in Vicon Motus using the direct linear transformation. The thorax-coxa (TC) joint angle was estimated by the angle created by the merus segment and the ventral carapace vector, with flexion moving the merus segment toward the rostrum of the animal. The angle of the coxa-basis (CB) joint was estimated by comparing the merus segment to the vertical carapace vector which extends vertically from the ventral carapace as a cross product of the lateral and ventral carapace vector. The $\mathrm{CB}$ joint was extended when the animal elevated the merus and flexed when the merus was depressed toward the substrate. Contact angle, lift angle, and angular excursions for each joint and DC angle were determined for each complete step for every leg during running. Angular excursions were the difference between the maximum and minimum angle during a step in the respective leg $\left(\theta_{\max ^{-}} \theta_{\min }\right)$.

\section{Results}

\section{Statistical analysis}

Statistical analyses were performed using SAS JMP 10 statistical software (SAS institute Inc.). A two-way nested ANOVA (Species X Trial Type) was performed on normalized $\Delta \mathrm{H}, \Delta \mathrm{V}$ and averaged velocity as well as joint excursion, contact and lift of angle for all 32 walking leg joints and the dactyl segments relationship to the substrate, followed by Tukey-Kramer multiple comparisons post-hoc tests ( $\alpha=.05)$.

\section{Body Structure and Stepping Pattern}

Since this was a comparative study, we began by looking at the basic body structure of the three species. Normalized to carapace width, all leg segments were significantly longer in 
ghost crabs than in Halloween crabs, with fiddler crabs mainly falling between the two $(p<0.05)$ (Fig. 3, Table 1). The dactyl was the only segment which was significantly longer in ghost crabs than either fiddler or Halloween crabs.

Each subsequent distal joint in the leg has a single plane of motion, either in the approximately horizontal plane (TC, CP, BM) or the approximately vertical plane (CB, $\mathrm{MC}$, PD)(Vidal-Gadea et al. 2007)(Fig. 2). Thus, the thorax-coxa (TC) joint flexes the leg rostrally and extends the leg caudally. The coxa-basis (CB) joint depress and elevate the leg, and the meruscarpus (MC) and propus-dactyl (PD) joint flex the distal segments under and extend distal segments away from the carapace.

All species of crabs adopted the same general locomotion strategy during sideways running. This general pattern is seen in the example of a ghost crab leading and trailing $1^{\text {st }}$ leg joint motion under control conditions (Fig 4). During the stance phase the leading legs flex the joints, pulling the carapace towards the legs, while the trailing legs extend the joints, pushing the carapace away from the legs. During the swing phase leading legs reach beyond the carapace in the direction of movement extending the joints while the trailing legs flex the joints toward the carapace. With a few exceptions all joints act in concert flexing and extending together during the respective leg's step and swing periods. Contralateral leg joints flex and extend in unison with their counterpart, except the CB joint which flexes the leg downward during stance on both sides.

Normalized stepping patterns show the three species each have distinct inter-leg coordination (Fig. 5). Fiddler crabs utilize an alternating tetrapod gait, with the L1/L3, L2/L4, T1/T3 and T2/T4 pairs of legs in phase with their partner. The L1/L3 legs are in phase with the T2/T4 legs and the L2/L4 legs are in phase with the T1/T3 legs during a stride. Ghost crabs display a similar semi-alternating tetrapod gait with no legs stepping differently from Fiddler crabs except for the leading and trailing $4^{\text {th }}$ legs. The $L 4$ leg had delayed stance onset $(p=0.0004)$ and was only used during $60 \%$ of strides. The T4 leg was only used during $36 \%$ of strides but had a similar stepping pattern to fiddler T4 leg $(p=0.79)$. Halloween crabs use a metachronal gait in which sequential stepping of each leg occurred in a wave pattern on both the trailing and leading side. This metachronal stepping pattern differs from the alternating tetrapod gait by delaying the L3, L4, T3, and T4 leg's stance initiation (all p<0.0002) (Fig. 3, Table 2). 


\section{Center of mass movement and Carapace rotation}

Species differences resulted in unique normalized center of mass movements in both the horizontal and vertical direction. Ghost crabs were the fastest animals with an average speed that was almost twice as fast as either of the two other species (Fig. 7, Table 3). Changes in center of mass height and velocity were greatest for the fiddler crabs and least for the Halloween crabs. Fiddler crab's substantial changes in center of mass movement created a bouncing 'stop and go' gait that had negative velocities for short periods during some strides. Ghost crabs had the fastest gait with intermediate changes in center of mass height and velocity, although it appeared that ghost crab velocity changes came from substantial thrust while fiddler crab's used both thrust and breaking. Halloween crabs were the slowest species with a consistent velocity and stable center of mass position (Fig. 6, Fig. 7, table 3).

Carapace rotations of roll and pitch showed significant species differences $(p<0.05)$, although yaw was similar across crab type $(p>0.05)$. Fiddler crabs produced the greatest roll and pitch excursions during a stride, although ghost crabs experienced similar pitch excursions. Halloween crabs had the lowest carapace rotations for pitch and roll, maintaining the most stable thorax of the three species (Fig. 8, Table 4).

\section{Walking Leg Joint Kinematics}

The three species of crab displayed very similar leg kinematics, with joint excursion, contact angle, and lift off angle similar for all 40 joints on all 8 walking legs except for seven differences (Table 5). Of the seven exceptions, six were differences within the two most proximal joints (TC and $\mathrm{CB}$ ) and one was within a distal joint (PD). There was no species difference in any of the $\mathrm{MC}$ joints, nor was there any difference in the dactyl position.

The leading legs of the animals showed three distinct differences in two joint's movement, including the L1 TC joint excursions and L3 CB excursions and lift off angle. Halloween and ghost crabs used the L1 TC joint to promote the leg in front of the animal during stance while fiddler crabs maintained the joint fairly stable during the course of a step (Fig. 9A/B and Table 5). This distinct L1 TC joint usage could possibly contribute to species differences in pitch excursion (Fig. 8B). The increased usage of the L1 TC joint may allow the lead first leg to support the rostral portion of the center of mass, decreasing pitch of the ghost and halloween crabs. 
The L3 CB excursions and lift off angle indicated that the three species may be using this joint during running for different purposes. Fiddler crabs seem to be using this joint to depress the leg, producing breaking and lift on the leading side of the carapace during stance. The joint builds angular momentum prior to stance producing impact upon contact and depressing the leg throughout the stance (Fig. 10C). This is emphasized by the substantial excursions and lower lift off angle (Fig.10A/B, Table 5) seen in the fiddler crab L3 CB joint. Ghost crabs appear to use this joint to buffer the breaking force produced by the leg, elevating the leg at contact and depressing the leg prior to lift off with the joint (Fig. 8C). Halloween crabs maintain the joint at a greater angle during stance (Fig. 10A/C), indicating the leg is in an elevated position in comparison to the other two crabs and may produce less breaking force in this position. These differences in the L3 $C B$ joint usage may be the reason for the excessive roll excursions and $\Delta$ Height seen in the fiddler crabs, as usage of this joint would increase upward lift on the leading side of the carapace. The L3 CB joint usage may also be partially responsible, in cooperation with trailing legs, for the greater average velocity and $\Delta$ Velocity seen in the ghost and fiddler crabs, respectively (Fig. 6, $7 A)$.

The trailing leg joints displayed four distinct differences in three joints, including the trailing $2^{\text {nd }}$ and $3^{\text {rd }}$ TC joint excursions, and the trailing $3^{\text {rd }}$ TC and PD joint contact angles. The trailing $2^{\text {nd }}$ and $3^{\text {rd }}$ TC joint appear to produce a substantial amount of thrust in the fiddler and ghost crabs, extending the joint during stance and flexing it during swing. Halloween crabs extend the joint during stance although the excursions during a step are not as substantial (Fig. 11, Table 5). This lack of trailing leg thrust and leading leg breaking (Fig. 10) could be responsible for the low $\Delta$ Velocity in the Halloween crabs. The Fiddler and ghost crabs appear to produce substantial thrust with the trailing legs, but the differential use of the Leading CB joint (Fig. 10) between the two species could be producing the differences seen in average velocity and $\Delta$ Velocity (Fig. 6, Fig. $7 A)$. The fiddler crab's greater excursions in the $L 3 C B$ joint and low lift angle could be producing breaking on the leading side which would cause the drastic changes in velocity seen in this species. And, although the fiddler and ghost crabs have very similar joint movements during thrust production, this difference in leading leg breaking movements could allow the ghost crabs to achieve a greater average velocity. 
Another difference in the trailing $3^{\text {rd }}$ TC joint use between species was contact angle, which was greater for ghost crabs (Fig. 12A, Table 5). This could be due to the lack of trailing $4^{\text {th }}$ leg usage during running, which was only used during $36 \%$ of strides in ghost crabs while the other crabs used the leg $100 \%$ of the time. The greater angle of this joint indicates that the ghost crabs maintain the trailing $3^{\text {rd }}$ leg in a posterior position, possibly widening the base of support during stance to compensate for the lack of T4 leg usage (Fig. 12B). Halloween crabs appear to compensate for the lack of TC joint usage in the $3^{\text {rd }}$ leg by decreasing T3 PD contact angle. This decrease in contact angle gives the joint a greater range of motion during stance, possibly allowing this joint to produce more thrust with a lower contact angle (Fig. 13, Table 5). This dependence on a distal joint with smaller muscles to produce thrust could be the reason the halloween crabs have the lowest average velocity (Fig. 6), even with a lack of breaking on the leading side due to $\mathrm{CB}$ joint position (Fig. 10).

The effect of mass on the movement of these species will be discussed in the subsequent paper (Chapter 3), although it is worth noting that the size of the animal was not associated with changes in joint behavior during running for any of the species.

\section{Discussion}

Structural differences in animals are the result of evolutionary pressures imposed by the properties of natural environments. Species specific locomotor patterns that overlay these body structures are also a property of the animals' environment, producing effective movement under the mechanical constraints of its body structure and habitat. Multiple species with similar body structures walk and run differently depending on native habitat including the camel and horse (Dagg, 1974), tokay gecko and leopard gecko (Aerts et al, 2000) as well as the goat and dog (Abourachid et al, 2007). Slight changes in neural control and limb morphology can permit these animals to move effectively in a variety of terrains by altering how limbs interact with the environment. The animals studied here utilized species specific morphology and interlimb coordination to create unique gaits with distinct body dynamics and running ability.

Fiddler crabs live in flat tidal areas sifting organic material from mud and do not often venture far from their burrow (Virgillio and Ribeiro, 2013). The flat tidal areas that fiddler crabs 
inhabit have a variety of locomotor demands due to tidal flooding. Periodic tidal water will increase buoyancy when animals are submerged and soften the terrain increasing substrate compliance. These challenges produced by their tidal environment may dictate an intermediate body design and allow fiddler crabs to move on muddy or hard terrain as well as while being submerged. The alternating tetrapod gait has previously been observed in fiddler crabs, although these researchers also recorded metachronal stepping patterns in the trailing legs during slow walk (Barnes, 1974). The reason this metachronal gait was not seen in the current study is because no slow walking was recorded, resulting in only alternating tetrapod gaits observed. Chinese mitten crabs tend to use alternating tetrapod stepping patterns like the fiddler crabs on smooth hard surfaces, but switch to random stepping patterns in rougher terrain. Chinese mitten crabs are also more likely to use a bouncing gait similar to the fiddler crab gait when on hard surfaces (Jianqiao et al, 2013). The periodic nature of the alternating tetrapod gait in fiddler crabs may be a product of running on a noncompliant track, resulting in large fluctuations in height and velocity during a stride. This bouncing alternating tetrapod gait may not persist if the running surface is more compliant or rough. It has also been shown that human legs modify mechanics in order to maintain center of mass movements when substrate compliance increases (Moritz and Farley, 2003). Although the crabs were transitioned to decreasing compliance, it would be expected that fiddler crabs would reduce vertical force output. Calculations show that increases in potential energy are greater than increases in kinetic energy for fiddler crabs, making vertical center of mass movements dominant over horizontal (Chapter 3). The joint activity observed in the leading legs would produce substantial vertical and breaking forces, and when combined with the thrust production of the trailing legs would result in a 'bouncing stop and go' gait when surface compliance is low and traction is high.

Ghost crabs move along the shoreline searching for carrion and predating on small animals (Wolcott, 1978). Having long limbs and a relatively narrow thorax allows these animals to achieve velocities of $2 \mathrm{~m} / \mathrm{s}$ and may create more effective movement during long distance running on open beaches. The relationship between long limbs and greater running speed has been observed in lizards (Bonine and Garland, 1999) and mammals (Garland and Janis, 1993). Long limbs would also provide more surface area for thrust in the fluid like sand (Chen et al, 
2013). Their long limbs may help with submerged locomotion by providing more surface area for paddling as well as longer limbs for 'underwater punting' which is a skipping motion performed underwater by shore crabs (Martinez et al, 1998). Both fiddler and ghost crabs experience the effects of neutral buoyancy and resistive fluid dynamics during underwater locomotion and may be the reason for their similar relative length in the proximal two leg segments.

Halloween crabs, on the other hand, are primarily terrestrial living along the coast line of tropical rainforests eating vegetation and leaf litter. They encounter more variety in terrain and obstacles than either of the other two species and are known to frequently climb in vegetation (Griffiths et al, 2007). Halloween crabs have the shortest leg segments and relatively widest carapace of the three species, possibly the reason these animals had the lowest relative velocity changes and center of mass movements during a stride. Halloween crabs also displayed the slowest normalized speed as well as the lowest $\Delta$ Velocity and $\Delta H$ eight, indicating that limb length is important for not only running speed but also movements of the body during a stride. These short limbs may be a product of their rainforest habitat in which climbing over obstacles and in foliage is more advantages than fast running. It has been shown that evolutionary tradeoffs exist within animal structure that determine maximal sprinting speed and climbing ability in chameleons (Losos et al, 1993) and lizards (Goodman, 2007; Lundelius, 1956). These same tradeoffs may be present in crab body structure, specializing decapods to either be effective runners or climbers which depends on limb structure and joint behavior.

Given that halloween crabs rarely become submerged in water (ref) and thus do not encounter periods of neutral buoyancy like fiddler and ghost crabs, having shorter limbs would allow for more effective terrestrial locomotion when relative body mass is greater. Shorter leg segments reduce leverage over joint movements allowing the same power to be used over a shorter distance. This greater torque production would promote terrestrial movement through a variety of terrains while increasing climbing ability. Morphology and movement analysis of arboreal and cursorial rodents has shown that limb length and joint usage can substantially alter torque production in specialized limbs (Stalheim-Smith, 1984). Furthermore, halloween crabs' relatively larger carapace and thick exoskeleton could be a defense against raccoons and other predators of their habitat and their short legs help provide support for heavier body structure. 
Although it is difficult to make definitive conclusions about the evolutionary pressures that created these species specific joint movements and body structures, it is apparent that the animals have developed locomotor strategies that allow for effective movement in their respective environments. This study was able to elucidate some of the effects of structural differences on locomotor capability and allow for an understanding of how these species' unique environments drive locomotor behavior. It has been shown that gait preference can determine body structure and joint usage in decapods (Vidal-Gadea et al, 2008) as well as neuromuscular activity (Dewell and Belanger, 2008). This study displayed that even with a similar preference for sideways running, body proportions and joint behavior can produce unique gaits. These structural and behavior differences resulted in unique mass specific energetic profiles and perturbation responses (Chapter 3). 


\section{References Cited}

Abdel-Aziz, Y. and H. Karara (1971) Direct linear transformation from comparator cooridnates into object space coordinates in close-range photogrammetry. ASP Symposium on Close Range Photogrammetry. Falls Church, VA: American Society of Photogrammetry.

Aerts, P., Damme, R., Vanhooydonck, B., Zaaf, A. and A Herrel (2000) Lizard locomotion: how morphology meets ecology. Netherlands Journal of Zoology. 50(2): 261-277

Abourachid, A., Herbin, M., Hackert, R., Maes, L. and V. Martin (2007) Experimental study of coordination patterns during unsteady locomotion in mammals. Journal of Experimental biology. 210: 366-372

Azizi, E. (2014) locomotor function shapes the passive mechanical properties and operating lengths of muscle. Proceedings of the Royal Society. 281: 2914-2921

Barnes, W. (1974) Leg co-ordination during walking in the crab, Uca pugnax. Journal of Comparative Physiology. 96: 237-256

Biewener, A. A. (1997) Effects of Elastic Energy Storage on Muscle Work and Efficiency. Journal of Applied Biomechanics, 13(4), 422-426

Bonine, K.E. and T. Garland (1999) Sprint performance of phynosomatid lizards, measured on a high-speed treadmill, correlates with hindlimb length. Journal of Zoology. 248(2), 255-265

Chen, L., Tingnan, Z. and D. Goldman (2013) A terradynamics of legged locomotion on granular media. Science. 339: 1408-1411

Dagg, A. (1974) The locomotion of the camel (Camelus dromedaries). Journal of Zoology. 174(1): 67-78

Dewell, R. and J. Belanger (2008) Degree of neuromuscular facilitation is correlated with contribution to walking in leg muscles of two species of crab. Journal of Comparative Physiology A. 194(12): 1031-1041

Doran, Diane M. (1993) Comparative locomotor behavior of chimpanzees and bonobos: the influence of morphology on locomotion. American Journal of Physical Anthropology. 92: 83-98

Full, J. R. and C. T. Farley (2000) Musculoskeletal dynamics in rhythmic systems: a comparative approach to legged locomotion. In, Biomechanics and neural control of posture and movement:New York: Springer Verlag

Jianqiao, L., Xiaodong, Z., Meng, Z., Rui, Z. and B. Chirende (2013) An experimental study on the gait patterns and kinematics of Chinese mitten crabs. Journal of Bionic Engineering. 10: 305-315 
Garland, T. and C. M. Janis (1993) Does metatarsal/femur ratio predict maximal running speed in cursorial mammals? Journal of Zoology. 229(1): 133-151

Goodman, B. (2007) Divergent morphologies, performance, and escape behavior in two tropical rock-using lizards (Reptilia: Scincidae). Biological Journal of the Linnean Society. 91(1): 8598

Griffiths, M.E, Mohammad, B.A and A. Vega (2007) Dry season distribution of land crabs, Gecarcinus quadrates (Crustacea: Gecarcinidae), in Corcovado National Park, Costa Rica. International Journal of Tropical Biology. 55: 219-224

Hill, A. V. (1950). The series elastic component of muscle. Royal society of London. Series B, Biological Sciences. 137(887): 273-80

Loeb, G. E (1995) Control implications of musculoskeletal mechanics. IEEE 17th Annual Conference, Volume: 2

Losos, J., Walton, B., and A. Bennett (1993) Trade-offs between sprinting and clinging ability in Kenyan chameleons. Functional Ecology. 7: 281-286

Lundelius, E. L. (1957) Skeletal adaptations in two species of sceloporus. Evolution. 11: 65-83

Jindrich, L. D. and R. J. Full (2002) Dynamic stabilization of rapid hexapedal locomotion. Journal of Experimental Biology. 205, 2803-2823

Josephson, Robert K. (1999). Dissecting Muscle Power Output. Journal of Experimental Biology. 202: 3369-75

Martinez, M., Full, R. and M. Koehl (1998) Underwater punting by an intertidal crab: a novel gait revealed by the kinematics of pedestrian locomotion in air versus water. Journal of Experimental Biology. 201: 2609-2623

Moritz, C. and C. Farley (2003) Human hopping on damped surfaces: strategies for adjusting leg mechanics. Proceedings of the Royal Society. Lond. 270: 1741-1746

Renous, S., Hofling, E. and V. Bels (2008) Locomotion patterns in two South American gymnophthalmid lizards: Vanzosaura rubric and Procellosaurinus tetradactylus. Zoology 111: 295-308

Stalheim-Smith, A. (1984) Comparative study of the forelimbs of semifossorial prarie dog, Cynomys gunnisoni, and the scansorial fox squirrel, Sciurus niger. Journal of Morphology. 180: $55-68$

Vidal-Gadea, A.G., Rinehart, M., and J. Belanger (2008) Skeletal adaptations for forwards and sideways walking in three species of decapod crustaceans. Arthropod Structure and Development. 37(2): 95-108 
Virgilio, A., P. Ribeiro (2013) Spatial and temporal patterns in the feeding behavior of a fiddler crab. Marine Biology. 160: 1001:1013

Wolcott, T.G (1978) Ecological role of ghost crabs, Ocypode quadrata (Fabricius) on an ocean beach: Scavengers or predators? Journal of Experimental Marine Biology and Ecology. 31(1): 67-82

Zajac, F.E (1989) Determining muscle's force and action in multi-articular movement. Exercise and Sport Sciences Reviews. 17:1, 187-230 


\section{Tables}

Table 1: Normalized Body Structure

\begin{tabular}{|c|c|c|c|c|c|c|}
\hline Segment & F-Ratio & Prob $>F$ & Species & $\begin{array}{l}\text { Length } \\
\text { (cm or } \\
\mathrm{CW})+/- \\
\text { error }\end{array}$ & Comparison & P-Value \\
\hline \multirow[t]{3}{*}{ Carapace } & \multirow[t]{3}{*}{ NA } & \multirow[t]{3}{*}{ NA } & Fiddler & $1.66 \pm 0.3$ & NA & NA \\
\hline & & & Ghost & $3.61 \pm 0.52$ & NA & NA \\
\hline & & & Halloween & $3.08 \pm 0.38$ & NA & NA \\
\hline \multirow[t]{3}{*}{ Merus } & \multirow[t]{3}{*}{3.56} & \multirow[t]{3}{*}{0.048} & Fiddler & $0.62 \pm 0.12$ & $F \times G$ & 0.48 \\
\hline & & & Ghost & $0.68 \pm 0.14$ & $\mathrm{~F} \times \mathrm{H}$ & 0.35 \\
\hline & & & Halloween & $0.55 \pm 0.04$ & $\mathrm{G} \times \mathrm{H}$ & $0.044^{*}$ \\
\hline \multirow{3}{*}{$\begin{array}{l}\text { Propus- } \\
\text { Carpus }\end{array}$} & \multirow[t]{3}{*}{6.74} & \multirow[t]{3}{*}{0.0075} & Fiddler & $0.63 \pm 0.08$ & $F \times G$ & 0.27 \\
\hline & & & Ghost & $0.71 \pm 0.14$ & $\mathrm{~F} \times \mathrm{H}$ & 0.14 \\
\hline & & & Halloween & $0.54 \pm 0.04$ & $\mathrm{G} \times \mathrm{H}$ & $0.006^{*}$ \\
\hline \multirow[t]{3}{*}{ Dactyl } & \multirow[t]{3}{*}{6.54} & \multirow[t]{3}{*}{0.0084} & Fiddler & $0.33 \pm 0.03$ & $F \times G$ & $0.017^{*}$ \\
\hline & & & Ghost & $0.45 \pm 0.11$ & $\mathrm{~F} \times \mathrm{H}$ & 0.99 \\
\hline & & & Halloween & $0.33 \pm 0.07$ & $\mathrm{G} \times \mathrm{H}$ & $0.014 *$ \\
\hline
\end{tabular}

Table 2: Normalized Stance Initiation

\begin{tabular}{|c|c|c|c|c|c|c|}
\hline Leg & F-Ratio & Prob $>F$ & Species & $\begin{array}{c}\text { Stance } \\
\text { Initiation }\end{array}$ & Comparison & P-Value \\
\hline \multirow[t]{3}{*}{ Leading $3^{\text {rd }}$} & \multirow[t]{3}{*}{4.68} & \multirow[t]{3}{*}{0.0001} & Fiddler & $0.06 \pm 0.13$ & $F \times G$ & 0.34 \\
\hline & & & Ghost & $0.02 \pm 0.14$ & $\mathrm{~F} \times \mathrm{H}$ & $0.0002^{*}$ \\
\hline & & & Halloween & $-0.15 \pm 0.2$ & $\mathrm{G} \times \mathrm{H}$ & 0.0663 \\
\hline \multirow[t]{3}{*}{ Leading $4^{\text {th }}$} & \multirow[t]{3}{*}{9.54} & \multirow[t]{3}{*}{0.0024} & Fiddler & $0.72 \pm 0.19$ & $F \times G$ & $0.0004^{*}$ \\
\hline & & & Ghost & $0.35 \pm 0.38$ & $\mathrm{FxH}$ & $<0.0001^{*}$ \\
\hline & & & Halloween & $0.28 \pm 0.22$ & $\mathrm{G} \times \mathrm{H}$ & 0.68 \\
\hline \multirow[t]{3}{*}{ Trailing 3rd } & \multirow[t]{3}{*}{9.24} & \multirow[t]{3}{*}{0.002} & Fiddler & $0.48 \pm 0.12$ & $F \times G$ & 0.48 \\
\hline & & & Ghost & $0.44 \pm 0.11$ & $\mathrm{~F} \times \mathrm{H}$ & $<0.0001^{*}$ \\
\hline & & & Halloween & $0.32 \pm 0.11$ & $\mathrm{G} \times \mathrm{H}$ & $0.002^{*}$ \\
\hline \multirow[t]{3}{*}{ Trailing $4^{\text {th }}$} & \multirow[t]{3}{*}{8.45} & \multirow[t]{3}{*}{0.0045} & Fiddler & $0.01 \pm 0.19$ & $F \times G$ & 0.79 \\
\hline & & & Ghost & $-0.19 \pm 0.33$ & $\mathrm{FxH}$ & $0.0016^{*}$ \\
\hline & & & Halloween & $-0.29 \pm 0.15$ & $\mathrm{G} \times \mathrm{H}$ & $0.0015^{*}$ \\
\hline
\end{tabular}


Table 3: Normalized Center of mass movement

\begin{tabular}{|c|c|c|c|c|c|c|}
\hline $\begin{array}{c}\text { Body } \\
\text { Rotation }\end{array}$ & F-Ratio & $\begin{array}{c}\text { Prob> } \\
\text { F }\end{array}$ & Species & $\mathrm{CW} \pm$ Error & Comparison & P-Value \\
\hline \multirow{3}{*}{$\begin{array}{l}\text { Average } \\
\text { Velocity }\end{array}$} & \multirow[t]{3}{*}{8.9} & \multirow[t]{3}{*}{0.005} & Fiddler & $11.7 \pm 2.9$ & $\mathrm{~F} \times \mathrm{G}$ & $0.0001^{*}$ \\
\hline & & & Ghost & $26.6 \pm 7.4$ & $\mathrm{~F} \times \mathrm{H}$ & 0.86 \\
\hline & & & Halloween & $9.4 \pm 3.6$ & $\mathrm{G} \times \mathrm{H}$ & $0.0001 *$ \\
\hline \multirow[t]{3}{*}{$\Delta$ Height } & \multirow[t]{3}{*}{6.0} & \multirow[t]{3}{*}{0.0132} & Fiddler & $0.28 \pm 0.15$ & $\mathrm{~F} \times \mathrm{G}$ & $0.02^{*}$ \\
\hline & & & Ghost & $0.19 \pm 0.09$ & $\mathrm{~F} \times \mathrm{H}$ & $0.0001^{*}$ \\
\hline & & & Halloween & $0.13 \pm 0.05$ & $\mathrm{G} \times \mathrm{H}$ & 0.09 \\
\hline \multirow[t]{3}{*}{$\Delta$ Velocity } & \multirow[t]{3}{*}{4.6} & \multirow[t]{3}{*}{0.03} & Fiddler & $9.45 \pm 4.3$ & $F \times G$ & 0.1 \\
\hline & & & Ghost & $6.7 \pm 5.3$ & $\mathrm{~F} \times \mathrm{H}$ & $0.000^{*}$ \\
\hline & & & Halloween & $4.4 \pm 2.8$ & $\mathrm{G} \times \mathrm{H}$ & 0.11 \\
\hline
\end{tabular}

Table 4: Carapace rotations

\begin{tabular}{|c|c|c|c|c|c|c|}
\hline $\begin{array}{c}\text { Body } \\
\text { Rotation }\end{array}$ & F-Ratio & $\begin{array}{c}\text { Prob> } \\
\text { F }\end{array}$ & Species & $\begin{array}{c}\text { Angle } \pm \\
\text { Error }\end{array}$ & Comparison & P-Value \\
\hline \multirow[t]{3}{*}{ Roll } & \multirow[t]{3}{*}{3.58} & \multirow[t]{3}{*}{0.054} & Fiddler & $15.3 \pm 21.8$ & $\mathrm{~F} \times \mathrm{G}$ & $0.023^{*}$ \\
\hline & & & Ghost & $7.9 \pm 4.7$ & $\mathrm{~F} \times \mathrm{H}$ & $0.0006^{*}$ \\
\hline & & & Halloween & $6.29 \pm 5.6$ & $\mathrm{G} \times \mathrm{H}$ & 0.58 \\
\hline \multirow[t]{3}{*}{ Pitch } & \multirow[t]{3}{*}{3.33} & \multirow[t]{3}{*}{0.058} & Fiddler & $14.3 \pm 9.1$ & $\mathrm{~F} \times \mathrm{G}$ & 0.65 \\
\hline & & & Ghost & $12.2 \pm 7.0$ & $\mathrm{~F} \times \mathrm{H}$ & $0.0068^{*}$ \\
\hline & & & Halloween & $6.3 \pm 5.6$ & $\mathrm{G} \times \mathrm{H}$ & 0.1 \\
\hline
\end{tabular}


Table 5: Joint kinematics

Table 5: ANOVA results for joint kinematics including excursions, contact and lift angles for all joints showing significance between species. Average angle +/- standard deviation as well as post-hoc comparisons and $p$-values are displayed.

\begin{tabular}{|c|c|c|c|c|c|c|c|}
\hline $\begin{array}{l}\text { Leg -- } \\
\text { Joint }\end{array}$ & $\begin{array}{c}\text { Measure } \\
\text { Angle }\end{array}$ & F-Ratio & $\begin{array}{c}\text { Probs } \\
\text { F }\end{array}$ & Species & $\begin{array}{c}\text { Angle } \pm \\
\text { Error }\end{array}$ & Comparison & P-Value \\
\hline \multirow[t]{3}{*}{$\mathrm{L} 1-\mathrm{TC}$} & \multirow[t]{3}{*}{ Excursion } & \multirow[t]{3}{*}{5.2} & \multirow[t]{3}{*}{0.018} & Fiddler & $12.5 \pm 7.4$ & $\mathrm{~F} \times \mathrm{G}$ & 0.29 \\
\hline & & & & Ghost & $18.0 \pm 9.7$ & $\mathrm{~F} \times \mathrm{H}$ & $0.012^{*}$ \\
\hline & & & & Halloween & $21.3 \pm 12.4$ & $\mathrm{G} \times \mathrm{H}$ & 0.91 \\
\hline \multirow[t]{3}{*}{ L3 -- CB } & \multirow[t]{3}{*}{ Excursion } & \multirow[t]{3}{*}{4.6} & \multirow[t]{3}{*}{0.028} & Fiddler & $31.3 \pm 13.8$ & $\mathrm{~F} \times \mathrm{G}$ & $<0.0001^{*}$ \\
\hline & & & & Ghost & $13.7 \pm 4.8$ & $\mathrm{~F} \times \mathrm{H}$ & $0.002^{*}$ \\
\hline & & & & Halloween & $17.7 \pm 9.2$ & $\mathrm{G} \times \mathrm{H}$ & 0.75 \\
\hline \multirow[t]{3}{*}{$\mathrm{L} 3-\mathrm{CB}$} & \multirow[t]{3}{*}{ Lift } & \multirow[t]{3}{*}{4.28} & \multirow[t]{3}{*}{0.0162} & Fiddler & $96.0 \pm 8.5$ & $F \times G$ & 0.35 \\
\hline & & & & Ghost & $88.4 \pm 7.9$ & $\mathrm{~F} \times \mathrm{H}$ & $<0.0001^{*}$ \\
\hline & & & & Halloween & $77.5 \pm 13.1$ & $\mathrm{G} \times \mathrm{H}$ & $0.056 *$ \\
\hline \multirow[t]{3}{*}{$\mathrm{T} 2-\mathrm{TC}$} & \multirow[t]{3}{*}{ Excursion } & \multirow[t]{3}{*}{3.76} & \multirow[t]{3}{*}{0.046} & Fiddler & $29.8 \pm 13.1$ & $F \times G$ & 0.99 \\
\hline & & & & Ghost & $\begin{array}{l}32.94 \\
\pm 16.4\end{array}$ & $\mathrm{~F} \times \mathrm{H}$ & $0.057^{*}$ \\
\hline & & & & Halloween & $\begin{array}{l}17.83 \\
\pm 9.18 \\
\end{array}$ & $\mathrm{G} \times \mathrm{H}$ & $0.026^{*}$ \\
\hline \multirow{3}{*}{ T3-TC } & \multirow{3}{*}{ Excursion } & \multirow{3}{*}{4.33} & \multirow{3}{*}{0.032} & Fiddler & $41.9 \pm 20.7$ & $\mathrm{~F} \times \mathrm{G}$ & 0.52 \\
\hline & & & & Ghost & $31.7 \pm 21.7$ & $\mathrm{~F} \times \mathrm{H}$ & $<0.0001^{*}$ \\
\hline & & & & Halloween & $\begin{array}{l}19.62 \\
\pm 16.5\end{array}$ & $\mathrm{G} \times \mathrm{H}$ & $0.036^{*}$ \\
\hline \multirow[t]{3}{*}{$\mathrm{T} 3-\mathrm{TC}$} & \multirow[t]{3}{*}{ Contact } & \multirow[t]{3}{*}{3.6} & \multirow[t]{3}{*}{0.054} & Fiddler & $67.5 \pm 16.5$ & $F \times G$ & $<0.0001 *$ \\
\hline & & & & Ghost & $97.1 \pm 24.4$ & $\mathrm{~F} \times \mathrm{H}$ & 0.8611 \\
\hline & & & & Halloween & $\begin{array}{l}72.86 \\
\pm 10.1\end{array}$ & $\mathrm{G} \times \mathrm{H}$ & $0.0002^{*}$ \\
\hline \multirow[t]{3}{*}{$\mathrm{T} 3-\mathrm{PD}$} & \multirow[t]{3}{*}{ Contact } & \multirow[t]{3}{*}{4.86} & \multirow[t]{3}{*}{0.023} & Fiddler & $\begin{array}{l}133.3 \\
\pm 19.1 \\
\end{array}$ & $F \times G$ & 0.99 \\
\hline & & & & Ghost & $\begin{array}{l}129.6 \\
\pm 12.0 \\
\end{array}$ & $\mathrm{~F} \times \mathrm{H}$ & $<0.0001 *$ \\
\hline & & & & Halloween & $\begin{array}{l}103.7 \\
\pm 14.1\end{array}$ & $\mathrm{G} \times \mathrm{H}$ & $0.0004^{*}$ \\
\hline
\end{tabular}




\section{Figures}

\section{Figure 1: Running Track}

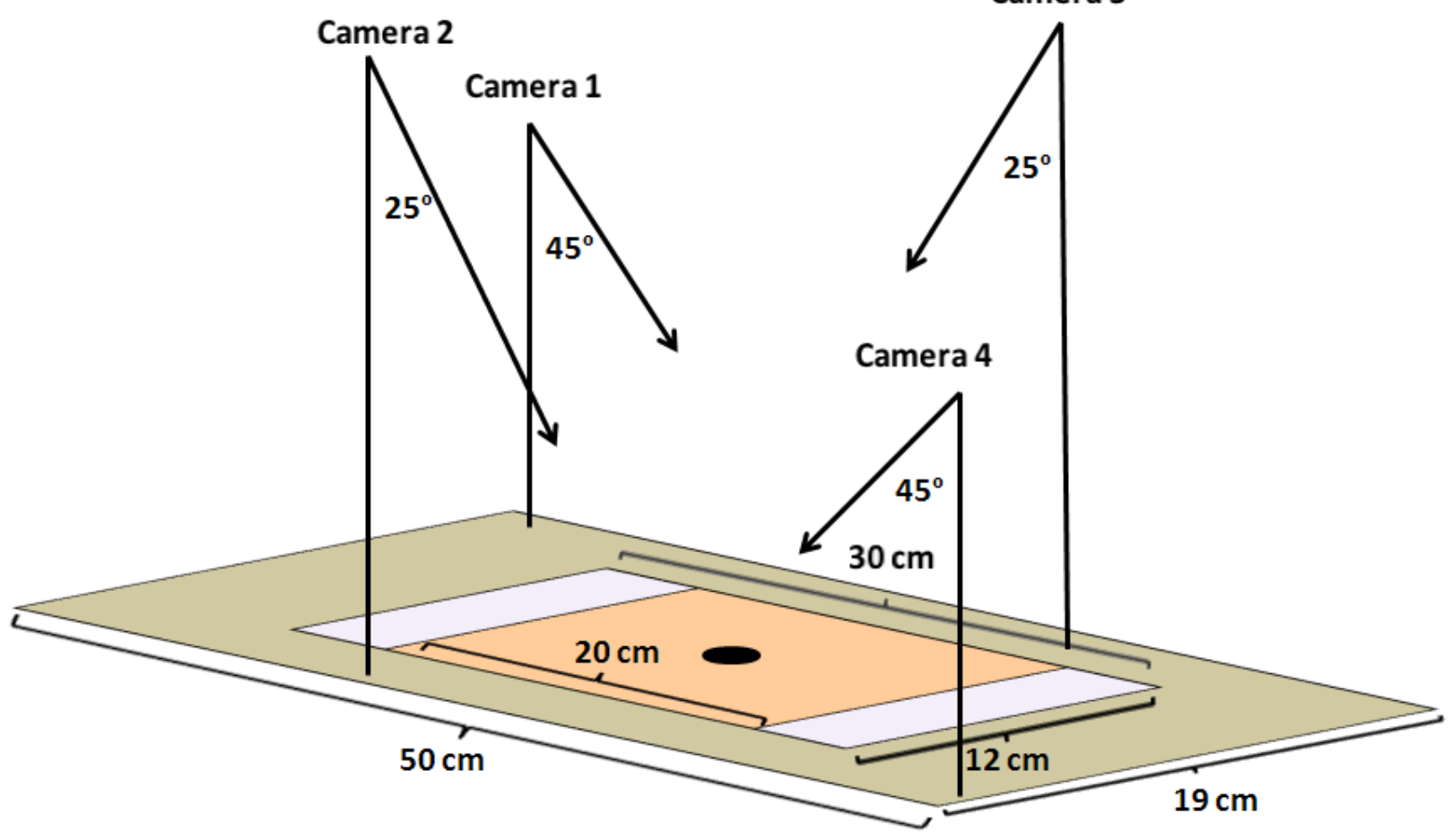

Figure 2: Diagram of the three running tracks and camera angles. The different tracks size is indicated by the variations in shading. All cameras were focused on the large black dot in the center and $100 \%$ of the track was captured in the video frame. Each track's length and width is indicated by the brackets in centimeters. 


\section{Figure 2: Walking Leg Joints}

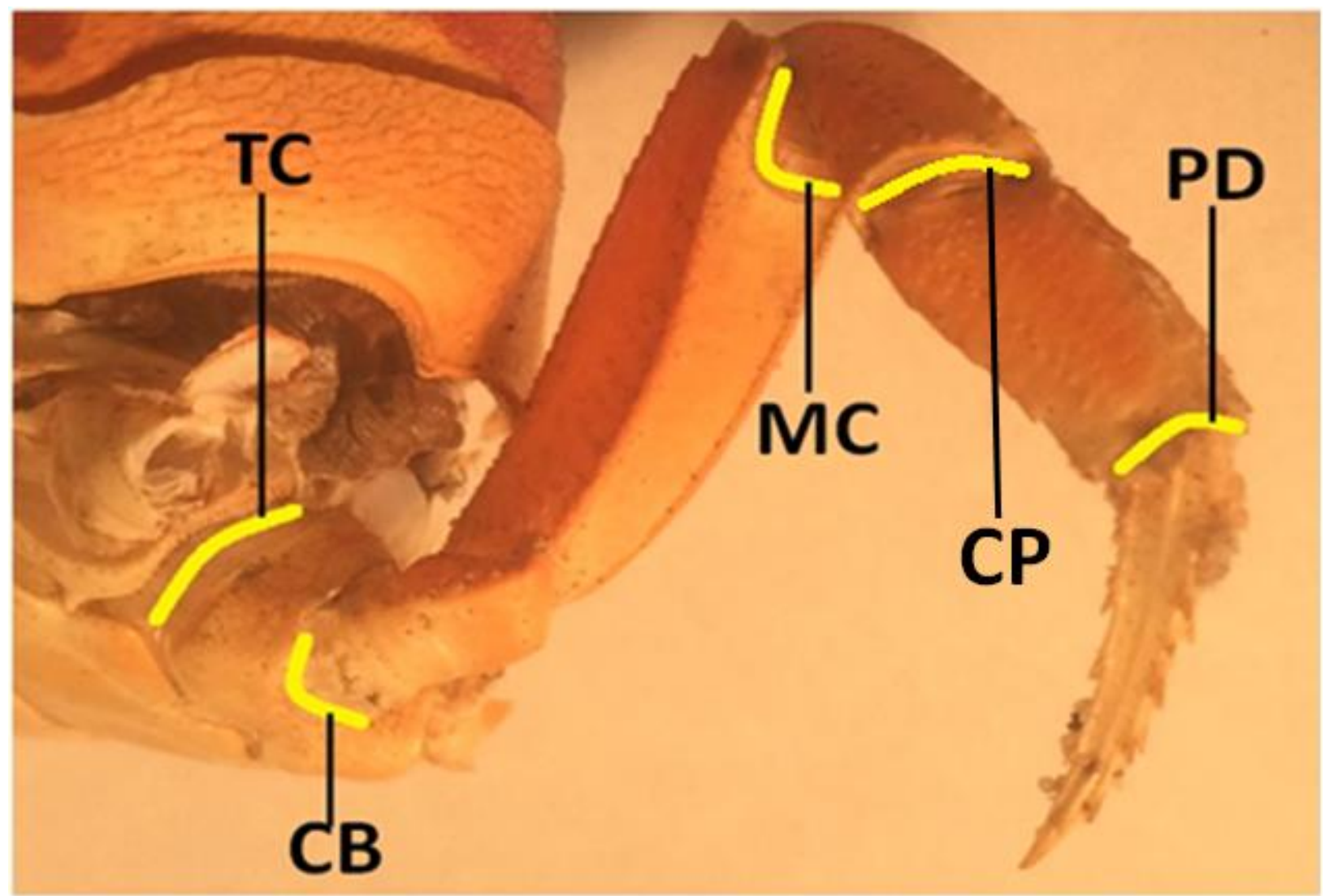

Figure 2: Walking leg joints highlighted in yellow. Joints moving in the horizontal plane are the thorax-coxa and carpus-propus joints (CP joint movement was not recorded due to its minimal use during locomotion. Vertical plane movement occurred in the coxa-basis, merus-carpus and propusdactyl joints. 
Figure 3: Body Structure

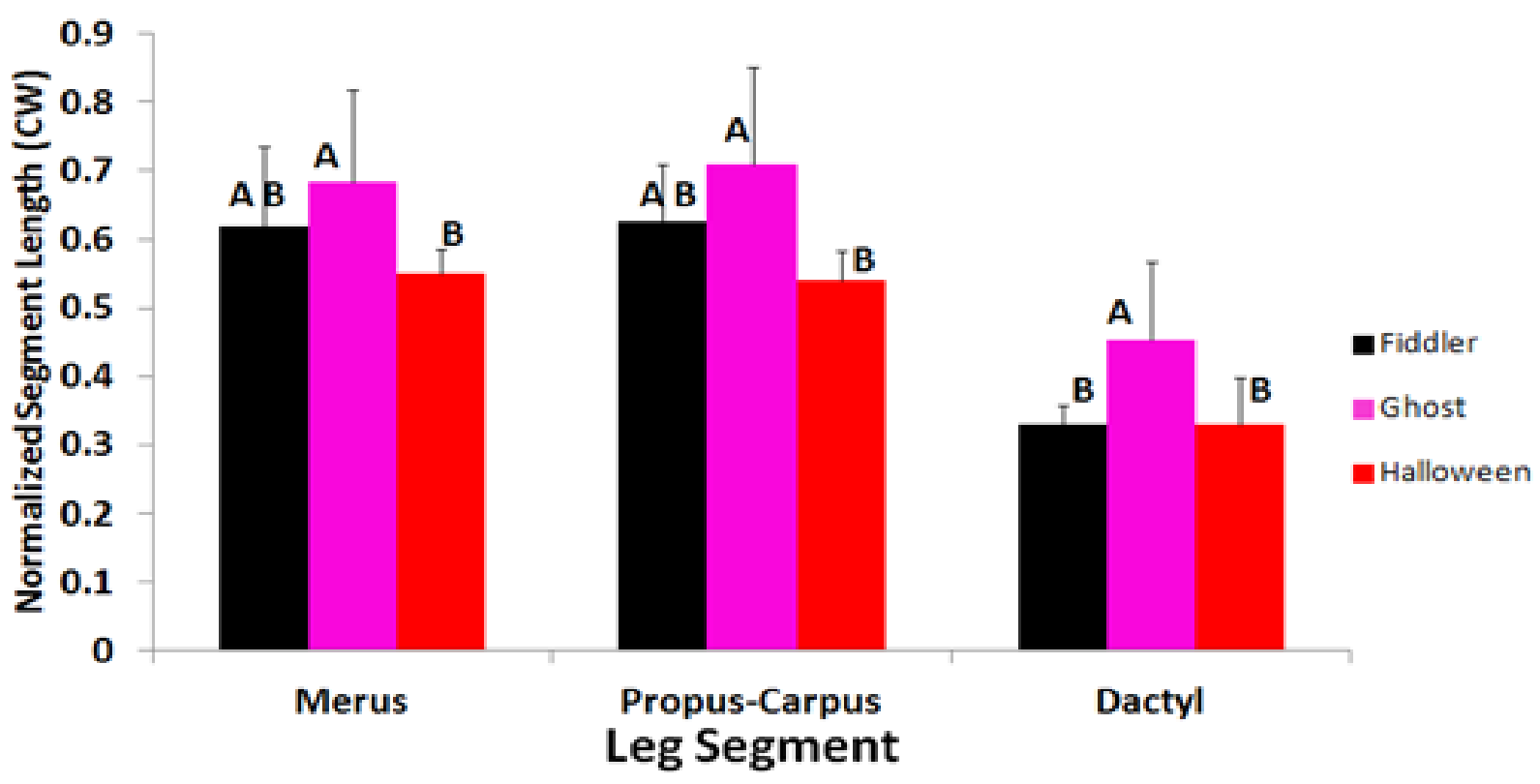

Figure 3: Body structure of the three limb segments monitored during running. Different letters above bars indicate segments that were significantly different in length compared to the other species when normalized to animals' carapace width. 
Figure 4: Leading and Trailing $1^{\text {st }}$ leg joint movement

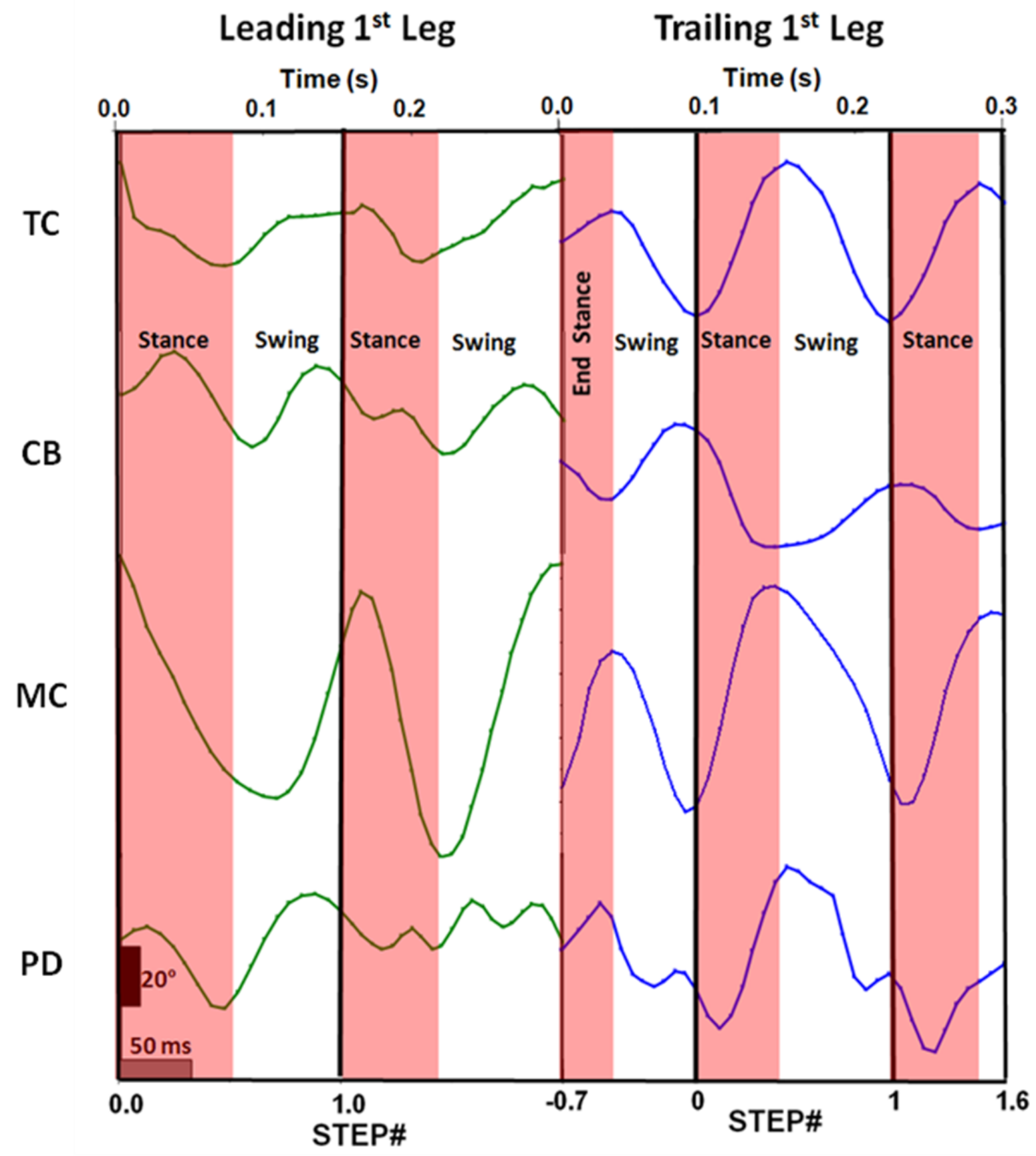

Figure 4: Joint movements of the leading and trailing $1^{\text {st }}$ leg during two steps by a ghost crab. The lower $x$-axis indicates the step, the upper $x$-axis indicates time and the $y$-axis indicates the joint. 


\section{Figure 5: Stepping Pattern}

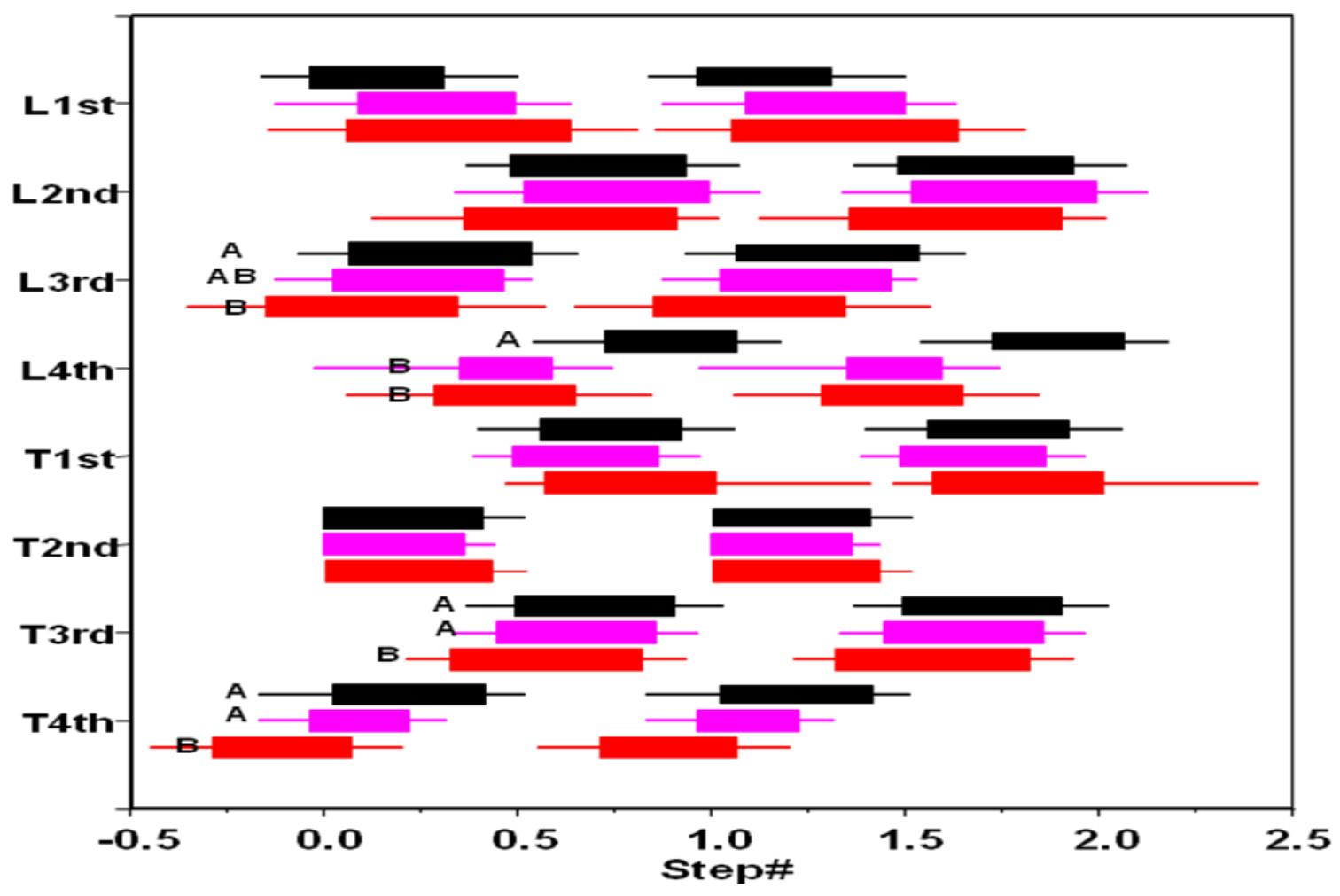

Figure 5: Stepping pattern for each leg normalized to stride (T2 step period). Different letters indicate which legs initiated stance at significantly different times between species. The left side of the bar indicates contact time in relation to stride and bar length indicates proportion of the stride the leg was in contact with the ground. Leading thin lines indicate standard deviation of stance onset, while trailing thin lines indicate standard deviation of stance duration. Species legend as in Figure 3. 
Figure 6: Normalized Velocity

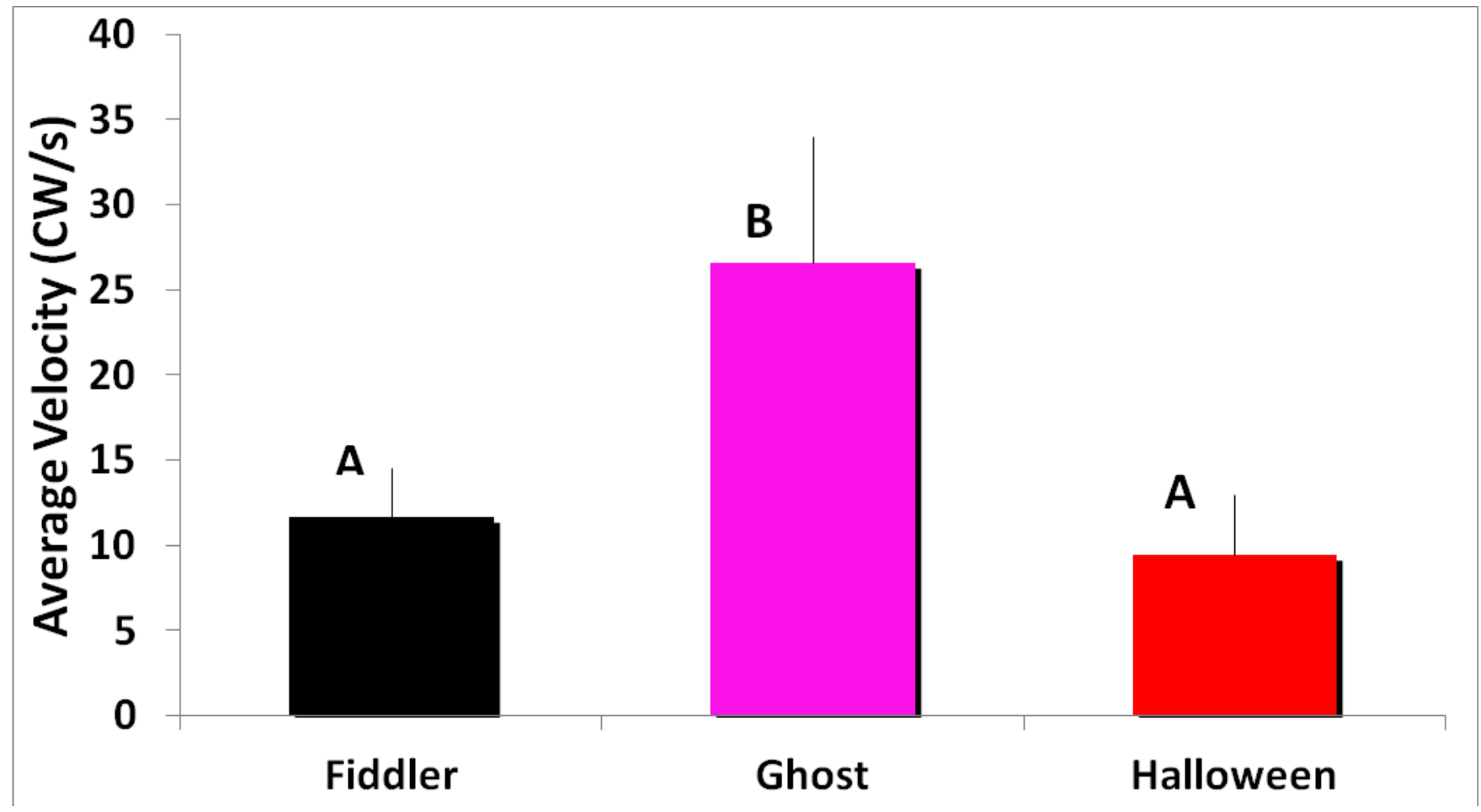

Figure 6: Average COM velocity normalized to carapace width. Letters indicate significantly different normalized velocities between species. Y-axis indicates average velocity throughout trial in carapace widths per second and upper lines indicate standard error. 
Figure 7: Center of mass movement
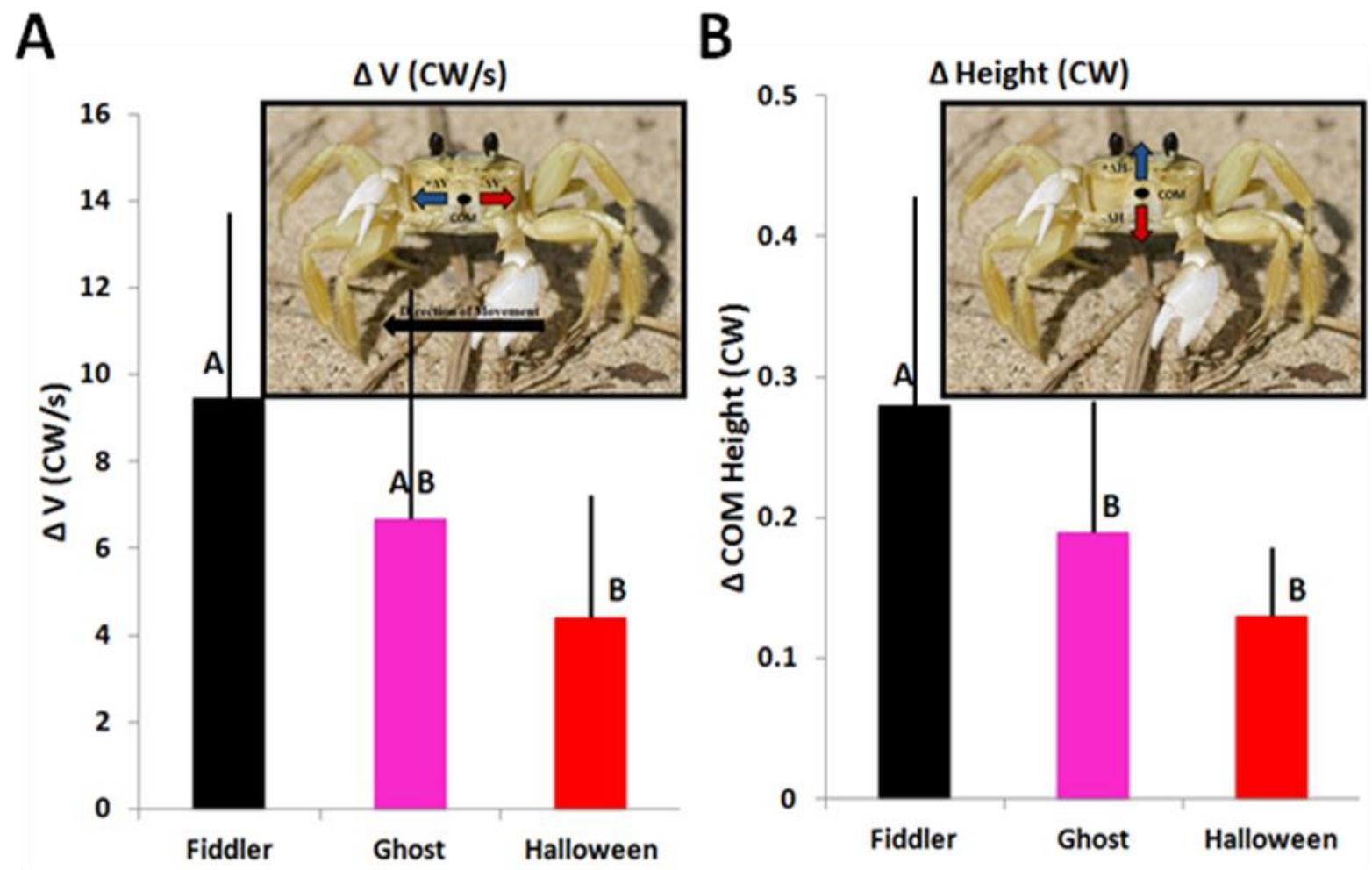

Figure 7: (A) Change in COM velocity during a stride. (B) Change in COM height during a stride. Bars represent averages and lines represent standard deviation. Letters indicate species that displayed significantly different COM movement during a stride. 
Figure 8: Carapace Rotations

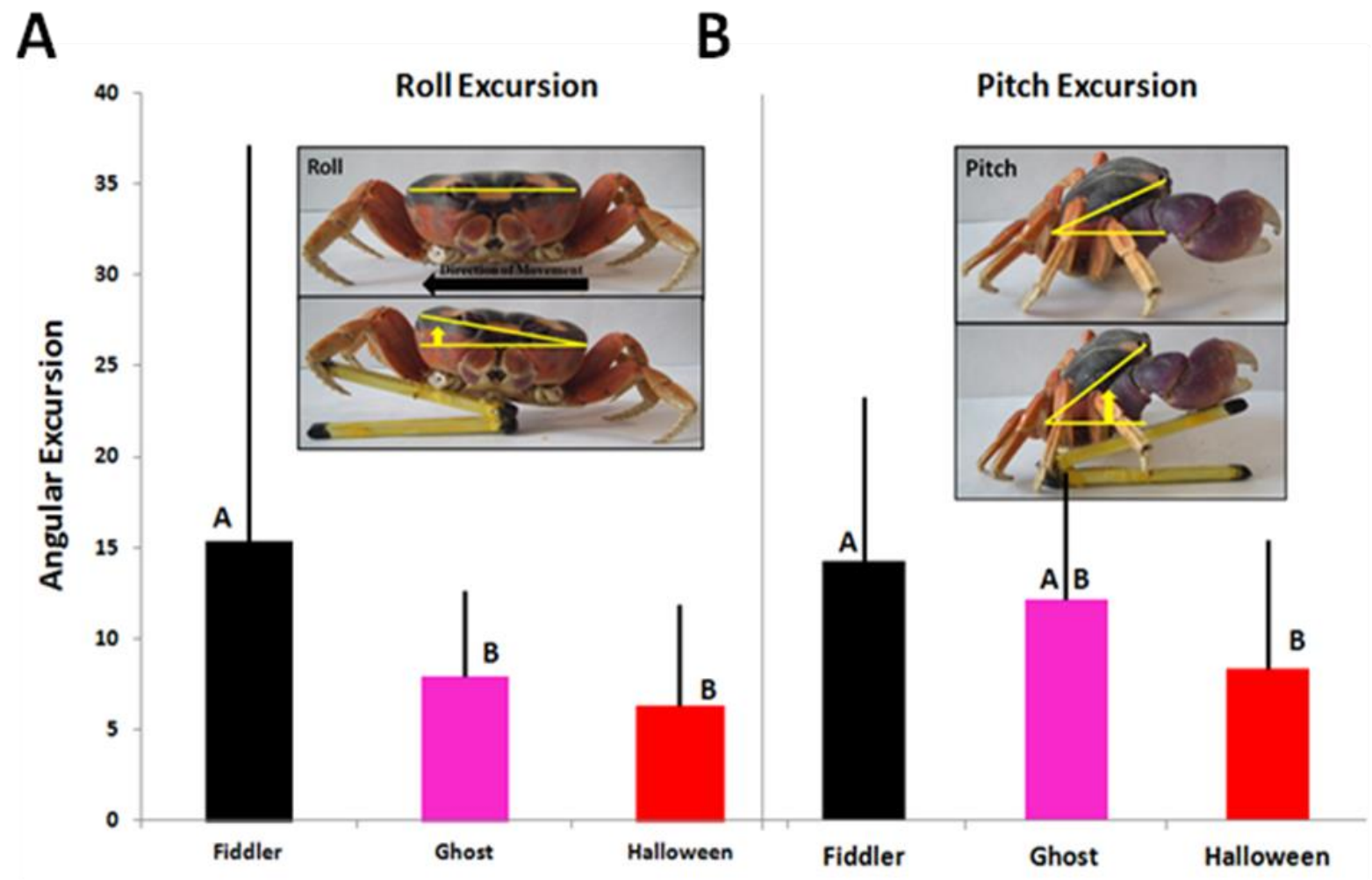

Figure 8: (A) Roll excursions for each species. (B) Pitch excursions for each species. Bars represent average excursion during a stride while lines represent standard error. Letters indicate which species were significantly different. $Y$-axis represents angular excursions in degrees and $\mathrm{X}$-axis represents species. 
Figure 9: Lead $1^{\text {st }}$ TC joint Excursions
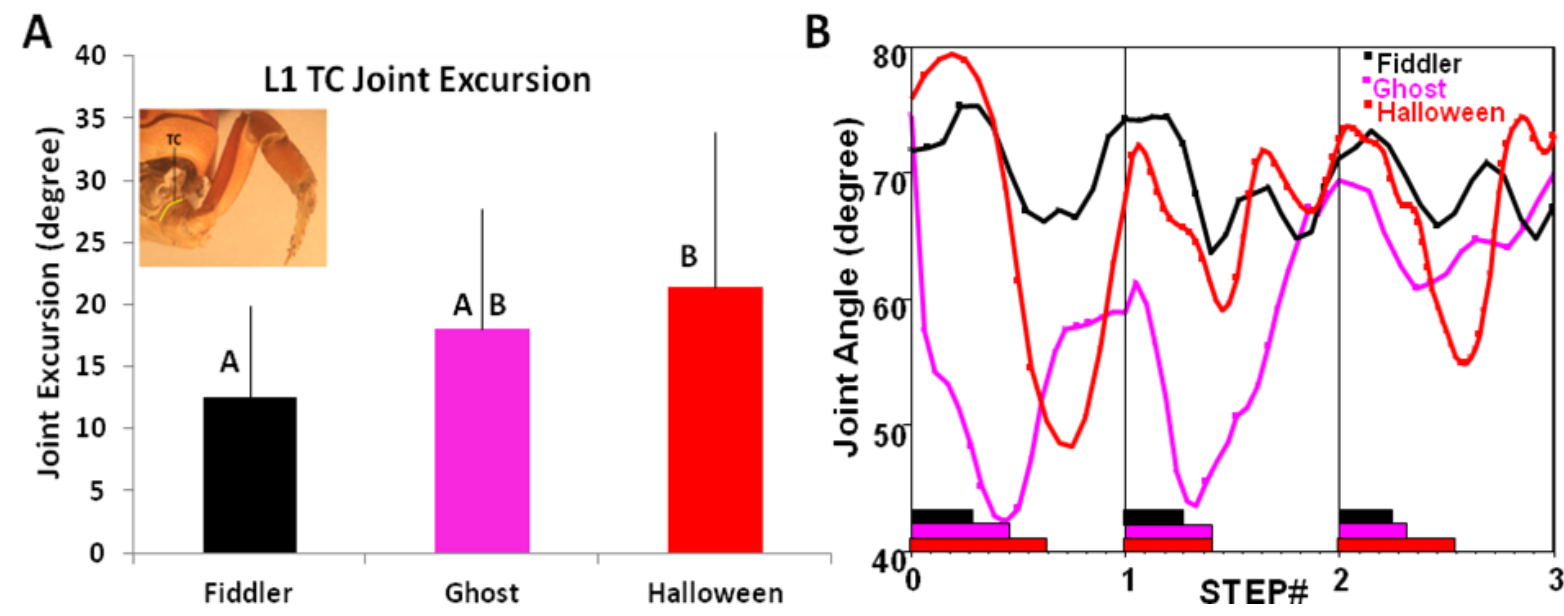

Figure 9: (A) Leading first thorax-coxa joint excursions for each species, letters indicate excursions that were significantly different between species. (B) Individual tracings of each joint during three complete steps, bars at the bottom represent proportion of step legs were in contact with the substrate. 
Figure 10: Lead $3^{\text {rd }}$ CB joint Excursion and Contact Lift
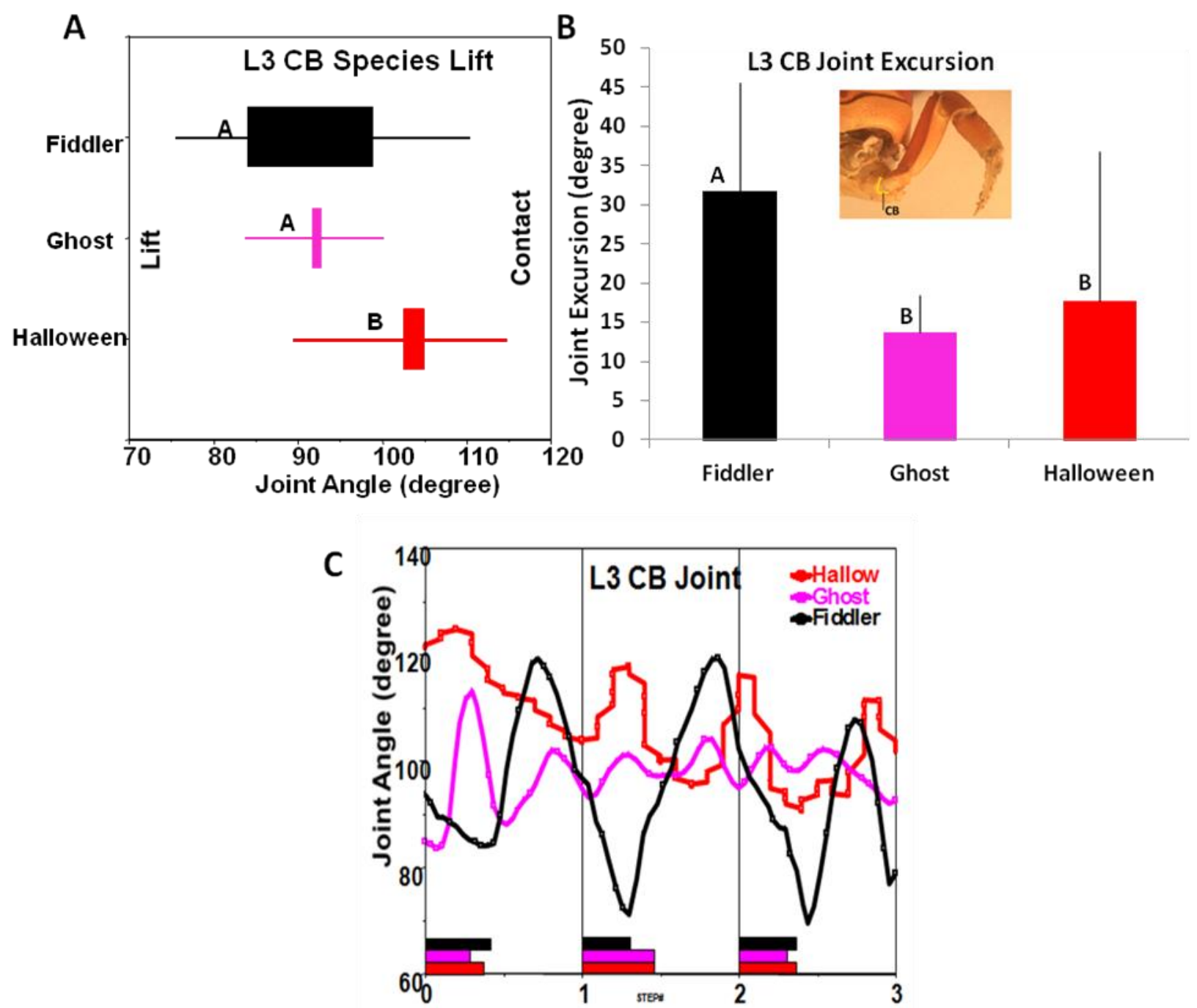

Figure 10: (A) Leading third coxa-basis joint lift angle comparison. $X$-axis represents joint angle in degrees and $y$-axis represents species. For this joint, the left side of the bars indicates lift angle and right side indicate contact angle while lines represent standard deviation. Letters represent lift angles that were significantly different. (B) L3 CB joint excursions with y-axis representing degrees and $x$-axis representing species. Letters indicate significantly different excursions between species. (C) Individual joint tracings of the L3 CB joint in the three species normalized to step period. X-axis indicates steps and $y$-axis indicates joint angle in degrees. Colored bars on the $x$-axis represent the proportion of the step the leg was in contact with the substrate. 
Figure 11: Trailing $2^{\text {nd }}$ and $3^{\text {rd }}$ TC joint excursions
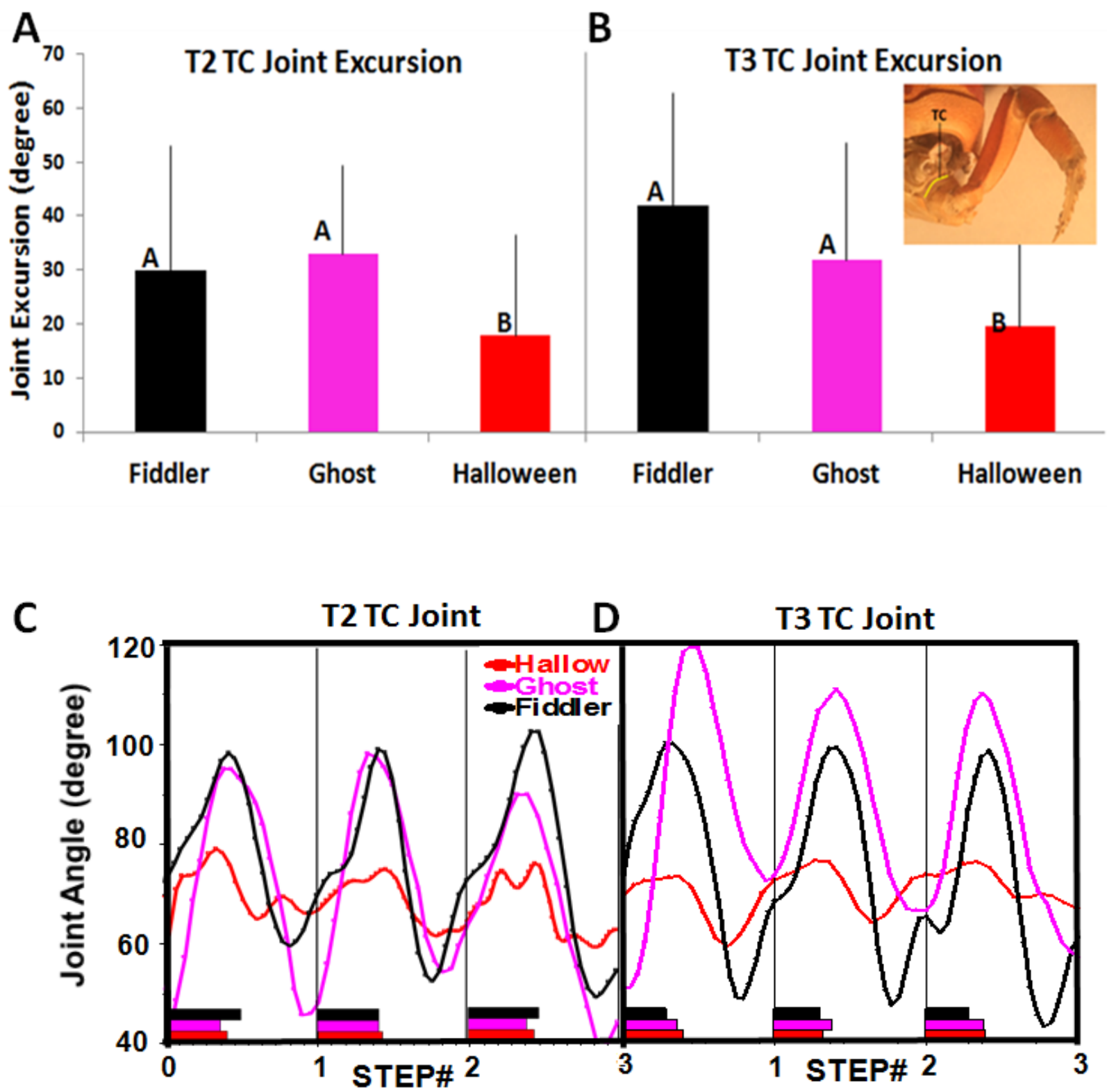

Figure 11: (A-B) Joint excursions during a step for T2 TC (A) and T3 TC (B) joints, bars indicate averages and lines indicate standard deviation. Species type is on the $x$-axis and joint excursion angle in degrees is on the y-axis. (C-D) Individual tracings of the T2 TC (C) and T3 TC (D) joints during three steps normalized to step period. $X$-axis represents the step number and $\mathrm{y}$-axis represents the joint angle in degree. Colored bars on the x-axis represent the proportion of each step the leg was in contact with the ground for each species. 
Figure 12: Trailing $3^{\text {rd }}$ TC joint Contact-Lift
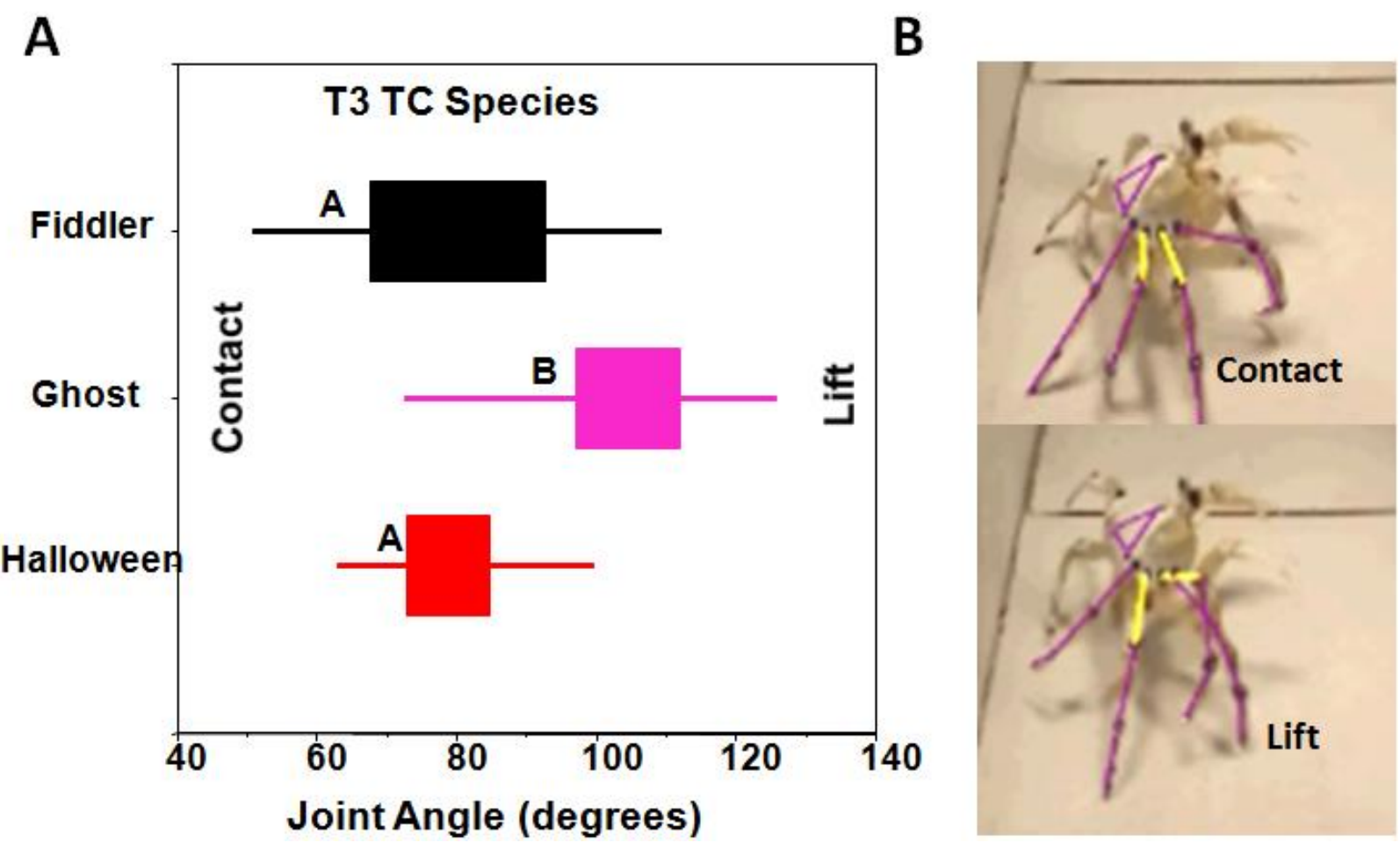

Figure 12: (A) Graph indicating the average contact angle of the T3 TC joint. X-axis represents joint angle at contact and lift while the $y$-axis represents species. (B) Image of ghost crab running with the T2 and T3 merus segment highlighted in yellow. Image displays the greater angle this species maintains in the T3 TC joint to compensate for the minimal use of the T4 leg. 
Figure 13: Trailing $3^{\text {rd }}$ PD Joint Contact

A

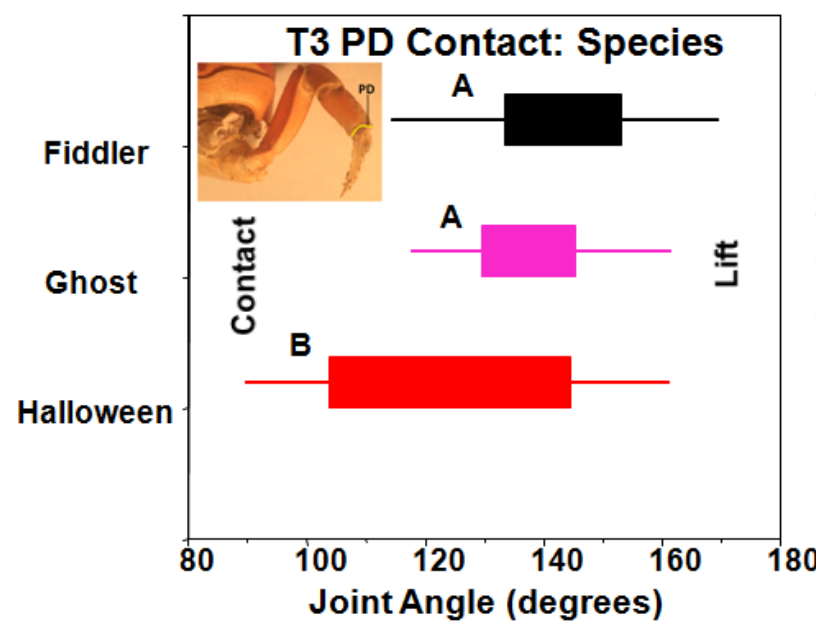

B

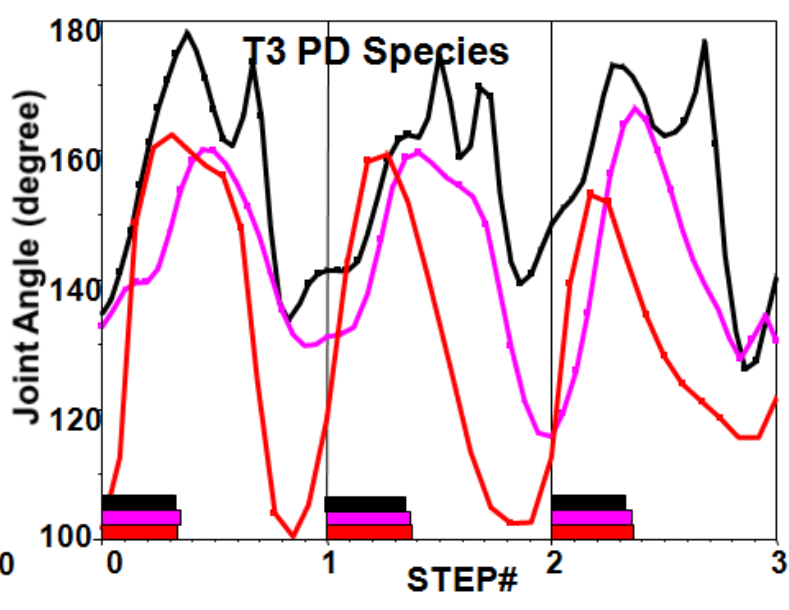

Figure 13: (A) T3 PD joint contact and lift angles. The left side of the bars indicates contact angle and the right side indicate lift angle in this leg. The letters represent contact angles that were significantly different between species. (B) Individual tracings of the T3 PD joint during three steps normalized to step time. Bars indicate proportion of step the leg was in contact with the substrate. The $x$-axis represents the steps and the $y$-axis represents the joint angle in degrees. 
Chapter 3: Locomotor Control Strategy Determines Stability during Perturbation Recovery in Three Species of Running Crabs 


\begin{abstract}
As locomotor speed increases, an organism becomes less reliant on sensory information due to the timing delays associated with neural feedback and muscle activation. Legged locomotion utilizes an animal's structure as well as the mechanical properties of the muscles and tendons to compensate for this lack of neural processing speed. It is not known how structural and overall size differences between species influence these mechanical properties. To further understand how size and structure affect the mechanics and stability of locomotion, three species of crab ranging from $2 \mathrm{~g}$ to $45 \mathrm{~g}$ were subjected to slippery surface perturbations while full body kinematics data was recorded. Each species displayed distinct responses to the perturbation using either static or dynamic stability to overcome the sudden loss of traction. The mechanical energetics of each species responded to the perturbation differently, altering the scaling effects associated with the mechanical energy recovery across animal weights. Distinct neuromechanical control and stability strategies are present in each species during unstable locomotion. These species specific neuromechanical strategies are built from the morphological and behavioral features of each animal, creating unique perturbation responses during running. Species specific gait dynamics dictate how stabilizing responses are performed during steps following a perturbation, utilizing the intrinsic mechanical and behavioral properties of the animals. Despite the differences in locomotor variables during perturbation recovery, it appears that animals utilize joint redundancy and/or abundant body momentum to produce stabilizing responses during perturbations.
\end{abstract}




\section{Introduction}

Locomotor stability in animals is a multidimensional problem that encompasses the mechanical properties of animals and the neural activity driving their movement, as well as the interaction of these variables with the environment (Nishikawa et al, 2007). Although many aspects of locomotor stability are known in uniform environments, it is beneficial to perturb locomotor systems away from the normal state to elucidate the stabilizing strategies used in natural environments. Multiple studies have used complex environments and mechanical perturbations to determine stability strategies, providing insight into the neuromechanical properties of animals during periods of instability (Jindrich and Full, 2002; Spongberg and Full, 2008; Daley et al, 2006; Daley et al, 2007; Clark and Higham, 2011). To continue this research, we sought to determine the mechanical variables associated with an unexpected change in substrata friction due to a slippery surface. We used three species of brachyuran crabs with differences in weight, body proportions and joint behavior. Although slippery surface perturbation studies have been conducted on bipedal animals (Clark and Higham, 2011), none have investigated the response to slippery surfaces in animals with the complexity of octopods. Furthermore, no studies have compared how differences in animal structure and size determine the response to a sudden loss of friction. The influence of traction on the movement of animals is an important area of research when determining factors that control stability and maneuverability during running because natural environments have non-uniform terrain with a range of friction coefficients (Alexander, 1982).

As animals increase the speed of movement, the time available to respond to perturbations decreases, forcing animals to rely on the mechanical properties of body structure instead of neural feedback (Koditschek et al, 2004), (Sponberg and Full, 2008). At high speeds the frequency of step cycles increases, reducing the time between strides. This time constraint makes neural feedback less useful, limiting the nervous systems ability to respond to changes in locomotor variables (Ting et al, 1994). As the nervous system becomes hindered by this lack of time, the mechanical behavior of an animal can perform some of the requirements of the nervous system. These mechanical properties produced by limb structure and muscle function integrate 
with neural processing to create a neuromechanical system that is able to produce stable movement (Nishikawa et al, 2007).

Two independent forms of locomotor stability are present in animals, static and dynamic stability (Karcnik, 2004). Static stability is prevalent in sprawled posture, slow moving animals that have at least three ground contact points supporting the center of mass (COM) throughout the gait. When the COM travels outside the perimeter of support, the animal becomes statically unstable and will fall (Full et al 2002). Many terrestrial locomotor strategies meet the statically stable criteria over a wide range of speeds, but when speed is increased the static stability of the system is reduced (Ting et al 1994). At increased speeds, many locomotor variables change, so that the criteria for static stability may no longer be satisfied due to the loss of constant COM support. Dynamic stability is used to bridge periods of static instability, utilizing body momentum to overcome the lack of static stability (Jindrich and Full 2002). Periods of static instability are particularly prevalent in animals that utilize fewer legs and/or aerial phases during locomotion, causing these animals to depend on dynamic stability throughout the gait (Full et al 2002).

Animals commonly encounter natural perturbations including uneven terrain, loss of friction, external forces and changes in substrate compliance, all of which deviate limb movement and COM trajectory. Analyzing the variables associated with perturbation responses is an informative approach to understanding how locomotion is controlled in natural environments. Control and recovery strategies have been shown in cockroaches experiencing unexpected forces (Jindrich and Full, 2002), guinea fowl and cockroaches running on uneven terrain (Daley et al, 2006; Sponberg and Full, 2008), and guinea fowl subject to a slippery surface during running (Clark and Higham, 2011). Although multiple species were used, one common theme has emerged from these perturbation studies. Supporting limbs must produce, absorb, store and release, and/or convert energy to achieve stability when mechanical patterns deviate during perturbations.

Given the multiple variables associated with locomotion and the complexity of perturbation recovery, models are useful when determining the underlying themes dictating 
stability. Models capable of capturing the variables associated with COM movements during locomotion provide a way of comparing animals with different structures and weights. These reductionist models, parsimoniously encode the movement of an animal, revealing the basic principles of motion without considering the finer details of body structure. The most prominent and widely used of these locomotor models is the spring-like inverted pendulum (SLIP) model for COM movement (Full and Koditschek, 1999). This SLIP model is composed of a pendulum and a spring in series, which are utilized in combination or individually. The SLIP model proposes a mechanism for minimizing energy expenditure through storing and recovering the energy during each phase of the step cycle. It explains two mechanisms for alternatively storing and recovering energy; first the pendulum effect of exchange between gravitational potential energy and kinetic energy and second the spring like effect due to exchanges of mechanical energy stored in muscles and tendons which is recovered as kinetic and gravitational energy (Cavagna et al, 1977).

Although the SLIP model can qualitatively reproduce the COM movement of running behavior in many locomotor designs, it is unable to capture the dynamic stability associated with the compliant structures of the musculoskeletal system. Still, the SLIP model is able to depict stable locomotion in a wide range of animals and provides a means of anchoring the complexity of locomotion in a uniform measure (Srinivasan and Holmes, 2007). The SLIP model has been applied to running brachyuran crabs, determining that the mechanical aspects of arthropod locomotion are mechanically similar to those of vertebrates despite the contrast in musculoskeletal structure (Blickhan and Full, 1987). The SLIP model will be applied to the three species of brachyuran crabs used here, enabling mechanical energy recovery to be compared across animal weights and species. These comparisons will further explain the effects of scaling and body structure during normal movement and during perturbation recovery.

While a good deal is known about the mechanics of running in uniform environments as well as basic perturbation responses, it is still unclear how differences in body structure and running behavior determine stability. The goal of this research was to determine the responses of three species of brachyuran crabs to a sudden loss of traction. These species were selected because they share similar body structure and movement direction but differ in weight range, 
relative limb length, and natural habitat. Quantifying the perturbation response of these crab species will allow for a better understanding of how mechanical and neural feedback differs between locomotor strategies during locomotion in non-uniform environments. We therefore tested the following hypotheses: (i) Utilization of static and dynamic stability will be reliant on the animal's relative speed. (ii) Animal structure, running behavior and weight will determine how the mechanical energy recovery system functions during normal running and perturbation recovery.

\section{Methods}

The experimental subjects and basic methods were as described in Chapter 2.

\section{Energy Calculations}

Energy calculations are adapted from Blickhan and Full (1987). Center of mass was determined for each species by hanging the animal by a string from three perpendicular points on the carapace. This point was then used to create a "virtual point" (as opposed to the marked points on the carapace and limbs) in the Vicon motion analysis software. The mass (M) of each animal with the COM velocity $(\mathrm{V})$ and height $(\mathrm{H})$ were used to calculate kinetic energy (KE $=1 / 2 \mathrm{MV}^{2}$ ) and potential energy $(\mathrm{PE}=\mathrm{MGH})$ where $\mathrm{G}$ is the acceleration due to gravity. Changes in kinetic and potential energies $(\triangle K E$ and $\triangle P E)$ were then calculated for each time interval throughout the two stride trial either during normal running (control) or slip recovery (perturbation). Change in COM energy $(\triangle \mathrm{CE})$ was calculated by summing the $\triangle K E$ and $\triangle P E$ for each time interval. Total kinetic $\left(\Sigma \Delta \mathrm{E}_{\mathrm{k}}\right)$, potential $\left(\Sigma \Delta \mathrm{E}_{\mathrm{p}}\right)$, and $\operatorname{COM}\left(\Sigma \Delta \mathrm{E}_{\mathrm{C}}\right)$ energy were calculated by summing the positive intervals for each over the course of the two strides. Mechanical energy recovery ratio was determined using the equation:

$\underline{\left(\sum \Delta \mathbf{E k}\right)+\left(\sum \mathbf{E p}\right)-\left(\sum \Delta \mathbf{E c}\right)}$

Recovery ratio $=\left(\sum \Delta \mathbf{E k}\right)+\left(\sum \Delta \mathbf{E p}\right)$ 
Mechanical energy recovered (MER) during each trial was determined by multiplying recovery ratio (RR) by the energy used by the $\operatorname{COM}\left(\Sigma \Delta \mathrm{E}_{k}+\sum \Delta \mathrm{E}_{\mathrm{p}}\right)$ to propel the animal down the track and maintain COM height (MER=RR $\left.\times\left(\sum \Delta \mathrm{E}_{\mathrm{k}}+\sum \Delta \mathrm{E}_{\mathrm{p}}\right)\right)$. Normalizing MER to weight (MER g1) provided a weightless representation of energy recovered (Blickhan and Full, 1987). The ratio of $\Sigma \Delta \mathrm{E}_{\mathrm{k}}$ to $\Sigma \Delta \mathrm{E}_{\mathrm{p}}$ was analyzed to determine the dominant energy form during normal running and to understand the effect of a perturbation on this relationship.

\section{Results}

\section{Statistical analysis}

Statistical analyses were performed using SAS JMP 10 statistical software (SAS institute Inc.). A two-way nested ANOVA (Species $X$ Trial Type) was performed on normalized $\Delta H, \Delta V$ and averaged velocity as well as joint excursion, contact and lift of angle for all 32 walking leg joints and the dactyl segments relationship to the substrate, followed by Tukey-Kramer multiple comparisons post-hoc tests $(\alpha=.05)$. Regression analysis was used to compare animal weight to MER and MER $\mathrm{g}^{-1}$ for each species during both trial types. An ANCOVA was used to determine the difference in MER and MER $\mathrm{g}^{-1}$ weight response for each species across trial type.

\section{Perturbation Recovery}

Mechanical energy recovered (MER) displayed distinct responses to weight in the three species. When normalized to weight, MER allowed for the efficiency of energy transfer between forward and vertical movement to be determined across animal sizes. Ghost and halloween crabs altered MER following a perturbation, while fiddler crab's MER was unaffected by a perturbation. These differences in energy recovery are due to the distinct perturbation response strategies of the three species, either harnessing kinetic energy or altering joint kinematics to regain stability.

\section{Ghost crab}

Ghost crabs appeared to use dynamic stability to recover from unexpected perturbations, regaining stability with the momentum from their relatively fast running gaits (Chap. 2). The 
crabs decrease velocity from $26.5 \mathrm{CW} / \mathrm{s}$ to $18.5 \mathrm{CW} / \mathrm{s}(\mathrm{p}=0.003$ ) (Fig. 2A)., and thus kinetic energy, in response to a perturbation which suggests a dynamically stable system that bridges periods of static instability with the body's momentum (Koditschek et al, 2004). This decrease in velocity reduced the ratio of total positive kinetic to potential energy changes from $2.7 \pm 1.5$ to $0.97 \pm 0.5$ $\left(\Sigma \Delta \mathrm{E}_{\mathrm{k}}: \Sigma \Delta \mathrm{E}_{\mathrm{p}}\right)(\mathrm{P}=0.02)$ (Table 3). This reduction in the total kinetic to potential energy ratio is demonstrated by a theoretical scenario of a SLIP model slowing down to half of the original speed but maintaining vertical COM movement (Fig. 2B). This scenario exemplifies how balancing kinetic and potential energy fluctuations can improve transfer between the two energy forms. This optimization of the SLIP model by slowing the COM down increases MER due to a change in attack angle of the virtual leg and center of mass (Fig. 2B). This increase in attack angle reduces leg compression and increases the amount of kinetic energy converted to potential energy due to the pendulum action of the center of mass and virtual leg. Ghost crabs have been shown to increase percent mechanical energy recovery as velocity decreases (Blickhan and Full, 1987). During perturbation recovery, larger crabs increased MER and normalized MER indicating that the larger animals' energy exchange was increased during the perturbation ( $p=0.05)$ (Fig. 1, Table 2). Velocity was the only variable to change during ghost crab perturbation recovery to explain the change in MER and normalized MER, except the increased probability of using the trailing $4^{\text {th }}$ leg during a stride (Fig. 2A) (Table 4).

Halloween crab

Halloween crabs decreased mechanical energy recovery in larger animals following a perturbation from $0.08 \mathrm{~mJ} / \mathrm{g}$ to $0.03 \mathrm{~mJ} / \mathrm{g}(\mathrm{p}=0.02$ ) (Fig. $2 \mathrm{~A}$, Table 2). Halloween crabs also decreased normalized energy recovery in larger animals following a perturbation from $7 \mathrm{E}^{-4} \mathrm{~mJ} / \mathrm{g}^{2}$ to $6 \mathrm{E}^{-5} \mathrm{~mJ} / \mathrm{g}^{2}(\mathrm{p}=0.016$ ) (Fig. $2 \mathrm{~B}$, Table 2). This indicates that Halloween crabs are only able to recover a certain amount of energy per unit weight during perturbed running, possibly due to the deteriorated conversion of kinetic to potential energy because of corrective movements.

Three different joint variables changed in Halloween crabs following a perturbation, including the L1 CB joint lift angle, T3 TC joint angular excursions, and T3 PD joint contact angle. The L1 CB joint decreased lift off angle following a perturbation, depressing the leg during stance 
through a greater range of angles ( $p=0.045$ ) (Fig. 4A). This shows that the Halloween crabs increase the support provided by the $\mathrm{L} 1 \mathrm{leg}$, and possibly could be altering the forward thrust provided by this $\mathrm{CB}$ joint. The individual tracings show that the animal contacted the substrate at a slightly lower L1 CB joint angle following a perturbation but flexed the joint at a greater proportional rate during stance, lifting the leg at a substantially lower L1 CB joint angle (Fig. 4B).

Halloween crab trailing $3^{\text {rd }}$ TC joint excursions increased following a perturbation (Fig. 5A) $(p=0.03)$, displaying that this once minimally active joint in comparison to the other species (Chapter 2) is now active during perturbation response. Individual tracing of the joint's angular movement displays that following a perturbation, the phase of joint movement changes and excursions increase in both directions along the horizontal plane (Fig. 5B). This change in joint movement appears to be in an attempt to place the leg in the most supportive position, extending and flexing the T3 TC joint to position the leg in rostral and caudal positions compared to normal running. Halloween crabs also increased T3 PD contact angle following a perturbation, indicating that the joint may be reducing the amount of thrust produced during perturbation recovery due to a decrease in joint movement during stance ( $p=0.023$ ) (Fig. 6A). Individual joint tracings show that the joint may not only reduce thrust following a perturbation, but also could produce breaking force during stance. During perturbation recovery the joint flexes upon contact which would result in breaking force on the trailing side of the animal, which has been documented in individual ghost crabs, although it is not the normal function of these legs (Blickhan and Full, 1987). This decrease in T3 PD usage and increase in L1 CB usage (Fig. 4) may imply that during a perturbation response the animals switch, at least in some leg joints, from thrust production to breaking or vice versa.

These changes in the joint movement of halloween crabs may reduce the amount of energy recovered, both on an entire animal and per gram basis due to the stabilizing effects on the center of mass, reducing the ability to convert kinetic to potential energy and vice versa. Interestingly the movements of the center of mass and average velocity of these animals did not change during perturbation recovery, indicating that other factors may play a role in the 
efficiency of the SLIP model including phase relationship and frequency of potential and kinetic energy changes.

\section{Fiddler crab}

Fiddler crabs maintained total energy recovered and normalized energy recovery across animal weights at control levels following a perturbation ( $p=0.46$ and $p=0.85$, respectively), indicating that these animals did not change the mechanism for kinetic to potential energy conversion. Each of the matched pair trials maintained very similar MER levels, indicating that the animals' energy recovery mechanisms were not altered by the perturbation. Normalized MER did not increase with animal weight, although the relationship with weight was significantly altered by the very large normalized MER in the smallest animal. This relationship between normalized MER and animal weight is probably produced by the relatively large fluctuations in forward velocity and COM height (Chapter 2), which would allow for a large amount of energy conversion between kinetic and potential energy. Furthermore, these animals had very low $\sum \Delta \mathrm{E}_{\mathrm{k}}$ $: \Sigma \Delta \mathrm{E}_{\mathrm{p}}$ ratio compared to the other animals due to their large potential energy fluctuations (Table 3). Although there is a large amount of energy available for conversion between the two energies, some animals were not able to achieve very high normalized MER. Even with large fluctuations in both forward velocity and vertical COM movement, MER will not occur unless the changes in energy are out of phase with one another. If kinetic and potential energy fluctuations are completely out of phase, it allows the animal maximum opportunity to conserve lost mechanical energy due to deceleration and gravity, respectively. The 'bouncing stop and go' gait of fiddler crabs does not adhere to this pattern of COM energy fluctuations as well as the other two species, with potential and kinetic energy fluctuations often occurring in phase with one another.

The perturbation response in fiddler crabs resulted in the change of three leading joint variables including L1 TC excursions as well as L3 and L4 MC joint contact angle. The animals increased L1 TC joint excursions to widen the range of leg placement in relation to carapace, positioning the leg in supportive positions depending on current carapace position and center of 
mass movement (Fig. 8A, Table 1). This movement in a joint that is normally inactive compared to the other species (chapter 2), implies that active control is being used, altering the motor output in this leg to provide stability. Individual joint tracing show regular movement of the joint with each step during control running, but following a perturbation the joint becomes highly variable with no real phase relationship during each step (Fig. 8B). The L3 MC joint decreases contact angle during perturbation recovery (Fig 9A, Table 1). This decrease is produced not by a change in overall joint excursions, but by a slight delay or advance of joint movement in relation to step phase. Individual joint tracings displays the joints range of movement following a perturbation are similar to the control trial; only the timing of joint movement during a step has changed (Fig 9. C). Fiddler crab L4 MC joint contact angle also decreased following a perturbation $(p=0.048)$ (Fig 9B, Table 1), although this MC joint change was due to altered leg position at contact. An individual tracing of the L4 MC joint during control and during an L3 leg slip event shows the leg is used on an 'as is' basis, catching the animal as it falls prior to full extension due to the L3 leg's loss of support. This resulted in a substantial decrease in contact angle during initial perturbation recovery, although the joint regains an approximation of normal movement in the subsequent steps (Fig. 9D).

\section{Discussion}

Brief perturbations caused by a slippery surface in running crabs produce stabilizing responses determined by animal size, body structure, and locomotor strategy. The three species of crab recovered from perturbations with distinct stability strategies, implementing dynamic and static stability through feedforward and feedback control mechanisms. These unique ensembles of stability and control strategies resulted in locomotor behaviors that are, despite the similarities in body layout and animal movement (Chap. 2), governed by very different mechanical principles.

Animal running speed determines gait selection (Alexander, 1984), running mechanics and stability (Koditschek et al, 2004). The effect of speed was seen during ghost crab perturbation recovery when dynamic stability predominated, slowing the crabs and thus changing the energetics of the gait. Although dynamic stability appeared to predominate during the ghost crab 
perturbation, static stability was evident in subsequent steps by the increased probability of the $4^{\text {th }}$ leg usage. This dynamic and static stability ensemble may allow ghost crabs to respond during unexpected perturbations, utilizing dynamic stability initially until momentum is reduced and static stability can dominate.

This reduction in velocity due to the dynamic stability response increased mechanical energy recovery by improving the mechanism for exchange between kinetic and potential energy (Farley et al, 1993). Ghost crabs move at a running gait during control trials, switching to a walking gait following the perturbation. Ghost crabs are known to recover a greater amount of mechanical energy when using a walking gait instead of a running gait. Larger crabs had a more substantial increase in mechanical energy recovery following a perturbation, possibly due to their heavier bodies or preferred walking speed (Blickhan and Full, 1987).

Halloween and fiddler crabs maintained their relatively slow speed and changed joint kinematics during a perturbation, implying that sensory feedback was altering motor output. This use of active control at relatively slow speeds indicates that halloween and fiddler crabs use static stability to recover from the perturbation (Ting et al 1994). Although static stability appears to dominate in these two species, guinea fowl and humans have been shown to maintain dynamic stability while maintaining body speed during slip perturbations. This is accomplished by keeping the path of the center of mass over the base of support (Clarke and Higham, 2011; You et al, 2001). The changes in these crabs' joint movements may have allowed for dynamic stability to be maintained by positioning them to support the center of mass.

Halloween crab leading $1^{\text {st }}$ leg changed its joint movement so as to provide greater support for the rostral portion of the body mass. This increase in stance angular excursions in a joint depressing the leg toward the substrate would provide lift on the leading side. Similarly, dairy cows alter step kinematics to increase vertical support during walking on low friction surfaces (Phillips and Morris, 2004). The trailing $3^{\text {rd }}$ leg of halloween crabs appear to switch from force producing to absorbing during perturbation recovery. The ability for a joint to act as a motor 
and a brake during running has been observed in coach roaches (Ahn and Full, 2001) and turkeys (Gabaldon et al, 2004).

Fiddler crabs altered three leading leg joints during perturbation response, indicating that the leading side limbs are important for stabilizing responses. Pigs differ joint loading in the forelimbs during slippery surface locomotion, possibly because these limbs are the primary weight supporters (Thorup et al, 2008). The leading limbs of ghost crabs have been shown to produce substantial breaking forces during locomotion (Blickhan and Full, 1987) and comparisons of joint kinematics suggest that breaking forces are greater in fiddler crabs (Chapter 2). These forces may make the support phase important when slippery surfaces are encountered by animals with leading limbs supporting substantial vertical and breaking forces.

Fiddler crab maintenance of mechanical energy recovery during perturbation response could be due to their 'bouncing stop and go' gait. The nature of the fiddler crab movement may make the animals less reliant on the pendulum activity and more reliant on spring component for mechanical energy conservation (Cavagna et al, 1977). Fiddler crab reliance on the spring like nature of the limbs could have made changes in the mass's pendulum like movement insignificant. Furthermore, the substantial pitch and role seen in these animals (Chapter 2) could indicate that a model encompassing lateral and rotational movement is also necessary (Full and Koditschek, 1999).

Although each crab species had a unique set of stability mechanisms, an overlying theme did emerge across all species. Animals appeared to use what resources were in excess during the perturbation; activating static joints, altering active joints or harnessing abundant body momentum to return the animal to a stable state. With 32 walking leg joints, each species of crab had distinct ensembles of movement during normal running (Chapter 2). With an excess of force producing and absorbing mechanisms some joints were not normally used, leaving redundancy in the system. The utilization of this redundancy is displayed by the altered joint movements in each of the species, activating normally inactive joints to regain stability. Furthermore, active 
joint movement was altered in the fiddler and halloween crabs which implies that the function of a joint can depend on the current stability of an animal. 


\section{References Cited}

Alexander, R. M. (1982) Locomotion of Animals: Glasgow: Blackie. pg 147

Ahn, A. and R. Full (2001) A motor and a brake: two leg extensor muscles acting at the same joint manage energy differ in a running insect. Journal ofExperimental Biology. 205: 379-389

Alexander, R.M (1984) Walking and Running. American Scientist. 72, 348-354

Allen, B. J. and J. S. Levinton (2007) Costs of bearing a sexually selected ornamental weapon in a fiddler crab. Journal of Functional Ecology. 21, 154-161

Blickhan, R., and R. J. Full (1987) Locomotion Energetics of the Ghost Crab II: Mechanics of the center of mass during walking and running. Journal of Experimental Biology. 130, 155-174

Cavagna, G.A., Heglund, N.C., and R. Taylor. (1977) Mechanical work in terrestrial locomotion: two mechanisms for minimizing energy expenditure. American Journal of Physiology. 233: 243-261

Clark, A. J., and T. E. Higham (2011) Slipping, sliding and stability: locomotor strategies for overcoming low-friction surfaces. Journal of Experimental Biology. 214: 1369-137

Daley, M. A., Usherwood, J. R., Felix, G. and A. Biewener (2006) Running over rough terrain: guinea fowl maintain dynamic stability despite a large unexpected change in subrate height. Journal Experimental Biology. 209: 171-187

Daley, M. A., Felix, G. and A. A. Biewener (2007) Running stability is enhanced by a proximo-distal gradient in joint neuromechanical control. Journal Experimental Biology. 210, 383-394

Farley, C.T, J. Glasheen and T. A. McMahon (1993) Running Springs: Speed and Animal Size. Journal of Experimental Biology. 185, pg 71-86

Full, R. J. (2002) Quantifying Dynamic Stability and Maneuverability in Legged Locomotion. Integrative and Comparative Biology. 42: 149-157

Full, R. and D. Koditschek. (1999) Templates and Anchors: Neuromechanical Hypotheses of Legged Locomotion on Land. Journal of Experimental Biology. 202: 3325-3332.

Gabaldon, A., Nelson, F., and T. Roberts (2004) Mechanical function of two ankle extensors in wild turkeys: Shifts from energy production to energy absorption during incline versus decline running. Journal Experimental biology. 207: 2277-2288

Jindrich, L. D. and R. J. Full (2002) Dynamic stabilization of rapid hexapedal locomotion. Journal of Experimental Biology. 205, 2803-2823

Karcnik, T. (2004) Stability in legged locomotion. Biological Cybernetics. 90: 51-58 
Koditschek, D. E., Full, R. J., and M. Buehler (2004) Mechanical aspects of legged locomotion control. Arthropod Structure and Development. 33: 251-272

Nishikawa, K., Biewener, A. A., Aerts, P., Ahn, A. N., Chiel, J. H., Daley, M. A., Daniel, T. L., Full, R. J., Hale, M. E., Hedrick, T. L., Lappin A. K., Nichols, R., Quinn, R. D., Satterlie, R. A., and B. Szymik. (2007) Neuromechanics: an integrative approach for understanding motor control. Integrative and Comparative Biology. 47: 16-54

Phillips, C., and I. Morris (2001) The locomotion of dairy cows on floor surfaces with different frictional properties. Journal Dairy Science. 84: 623-628

Sponberg, S. and R. J. Full. (2008) Neuromechanical response of musculo-skeletal structures in cockroaches during rapid running on rough terrain. Journal of Experimental Biology. 211: 433-446

Srinivasan, M. and P. Holmes (2007)How well can spring-mass-like telescoping leg models fit multi-pedal sagittal-plane locomotion data? Journal of Theoretical Biology. 255, 1-7

Thorup, V., Laursen, B. and B. Jensen (2008) Net joint kinetics in the limbs of pigs walking on concrete floor in dry and contaminated conditions. Journal of Animal Science. 86: 992-998

Ting, L. H., Blickhan, R., and R. J. Full. (1994) Dynamic and static stability in hexapedal runners. Journal of Experimental. Biology. 197: 251-269

Walls, M.L. and J.E. Layne (2009) Fiddler Crabs Accurately Measure Two-Dimensional Distance Over ThreeDimensional Terrain. Journal of Experimental Biology. 212: 3236-3240

You, J., Chou, Y., Lin, C., and F. Su (2001) Effect of slip on movement of body center of mass relative to base of support. Clinical Biomechanics. 16: 167-173 


\section{Tables}

Table 1: Joint Kinematics: Perturbation

\begin{tabular}{|c|c|c|c|c|c|c|c|}
\hline Species & $\begin{array}{l}\text { Leg -- } \\
\text { Joint }\end{array}$ & $\begin{array}{c}\text { Measure } \\
\text { Angle }\end{array}$ & F-Ratio & Prob> F & $\begin{array}{c}\text { Control } \\
\text { Angle } \pm \\
\text { Error }\end{array}$ & $\begin{array}{c}\text { Perturbation } \\
\text { Angle } \pm \\
\text { Error }\end{array}$ & P-Value \\
\hline \multirow[t]{3}{*}{ Halloween } & $\mathrm{L} 1-\mathrm{CB}$ & Lift & 3.21 & 0.044 & $61.2 \pm 15.8$ & $47.2 \pm 15.1$ & $0.045^{*}$ \\
\hline & $\mathrm{T} 3-\mathrm{TC}$ & Excursions & 4.33 & 0.0317 & $19.6 \pm 16.5$ & $30.6 \pm 16.2$ & $0.033^{*}$ \\
\hline & T3 - PD & Contact & 4.86 & 0.023 & $103.7 \pm 14.1$ & $123.1 \pm 26.9$ & $0.008^{*}$ \\
\hline \multirow[t]{3}{*}{ Fiddler } & L1 -TC & Excursion & 5.16 & 0.019 & $12.5 \pm 7.42$ & $19.5 \pm 15.5$ & $0.056^{*}$ \\
\hline & $\mathrm{L} 3-\mathrm{MC}$ & Contact & 3.8 & 0.05 & $141.2 \pm 11.3$ & $119.8 \pm 16.8$ & $<0.0001^{*}$ \\
\hline & $\mathrm{L} 4-\mathrm{MC}$ & Contact & 3.7 & 0.048 & $136.6 \pm 13.8$ & $119.4 \pm 18.8$ & $0.004^{*}$ \\
\hline
\end{tabular}

Table 2: Mechanical Energy Recovery

\begin{tabular}{|c|c|c|c|c|c|c|}
\hline \multirow[t]{2}{*}{ Species } & \multirow[t]{2}{*}{ T-ratio } & \multirow[t]{2}{*}{ Prob $>T$} & \multicolumn{2}{|c|}{ Control Runs } & \multicolumn{2}{|c|}{ Perturbation Runs } \\
\hline & & & $\begin{array}{c}\text { Weight } \\
\text { Response }\end{array}$ & $R^{2}$ & $\begin{array}{c}\text { Weight } \\
\text { Response }\end{array}$ & $R^{2}$ \\
\hline Fiddler (mJ/g) & 0.84 & 0.46 & 0.07 & 0.75 & 0.10 & 0.71 \\
\hline $\begin{array}{c}\text { Norm. Fiddler } \\
\left(\mathrm{mJ} / \mathrm{g}^{2}\right)\end{array}$ & 0.2 & 0.85 & $7 \mathrm{E}^{-3}$ & 0.27 & $6 \mathrm{E}^{-3}$ & 0.48 \\
\hline Ghost (mJ/g) & 2.96 & $0.02 *$ & 0.056 & 0.60 & 0.2 & 0.86 \\
\hline $\begin{array}{c}\text { Norm. } \\
\text { Ghost }\left(\mathrm{mJ} / \mathrm{g}^{2}\right)\end{array}$ & 2.27 & $0.056^{*}$ & $9 \mathrm{E}^{-5}$ & 0.01 & $2 E^{-3}$ & 0.70 \\
\hline $\begin{array}{c}\text { Halloween } \\
(\mathrm{mJ} / \mathrm{g})\end{array}$ & -2.96 & $0.02 *$ & 0.08 & 0.85 & 0.03 & 0.86 \\
\hline $\begin{array}{c}\text { Norm. } \\
\text { Halloween } \\
\left(\mathrm{mJ} / \mathrm{g}^{2}\right)\end{array}$ & -3.03 & $0.016^{*}$ & $7 \mathrm{E}^{-4}$ & 0.77 & $6 \mathrm{E}^{-5}$ & 0.10 \\
\hline
\end{tabular}


Table 3: Kinetic: Potential Energy ratio

\begin{tabular}{|c|c|c|c|c|}
\hline \multirow[t]{2}{*}{ Species } & \multicolumn{2}{|c|}{ Mean KE: PE ratio \pm Error } & \multirow[t]{2}{*}{ Z- value } & \multirow[t]{2}{*}{ P-Value } \\
\hline & Control & Perturbation & & \\
\hline Fiddler & $0.46 \pm 0.24$ & $0.49 \pm 0.28$ & -0.24 & 0.81 \\
\hline Ghost & $2.73 \pm 1.46$ & $0.97 \pm 0.51$ & 2.32 & $0.02 *$ \\
\hline Halloween & $1.30 \pm 1.63$ & $0.41 \pm 0.29$ & 1.36 & 0.17 \\
\hline
\end{tabular}

Table 4: Probability of $4^{\text {th }}$ walking leg step during stride

Table 4- Probability of $\mathbf{4}^{\text {th }}$ walking leg during a stride: The probability of using the trailing and leading $4^{\text {th }}$ walking leg during a stride for each species is displayed for both control and perturbation running.

\begin{tabular}{|c|c|c|c|c|}
\hline \multirow{2}{*}{ Species } & \multicolumn{2}{|c|}{ Lead 4 $^{\text {th }}$ leg Step probability } & \multicolumn{2}{c|}{${\text { Trailing } \text { 4 }^{\text {th }} \text { leg Step Probability }}$} \\
\cline { 2 - 5 } & Control & Perturbation & Control & Perturbation \\
\hline Fiddler & 1 & 1 & 1.05 & 1 \\
\hline Ghost & 0.6 & 0.6 & 0.4 & 0.8 \\
\hline Halloween & 1.1 & 1 & 1.1 & 1 \\
\hline
\end{tabular}




\section{Figures}

Figure 1: Ghost crab mechanical energy return
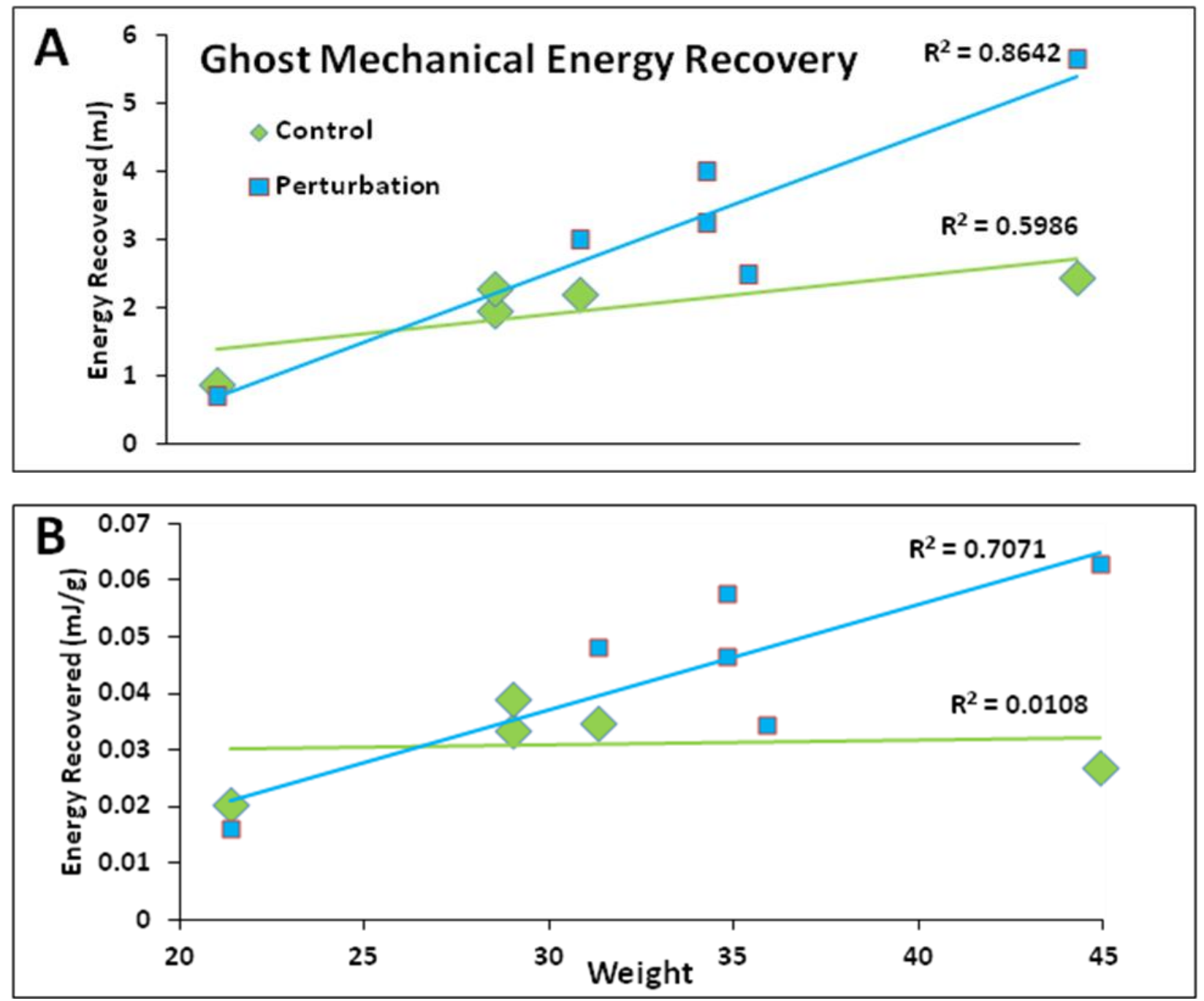

Figure 1- Ghost crab mechanical energy recovered and normalized mechanical energy recovered: Regression analysis of mechanical energy recovered (A) and normalized to weight (B) across weight ranges of animals studied. $\mathrm{Y}$-axis displays energy in $\mathrm{mJ}$ and $\mathrm{X}$-axis displays ghost crab weight in grams, $\mathrm{R}^{2}$ values are displayed for each trial type. 
Figure 2: Ghost Crab perturbation response: velocity
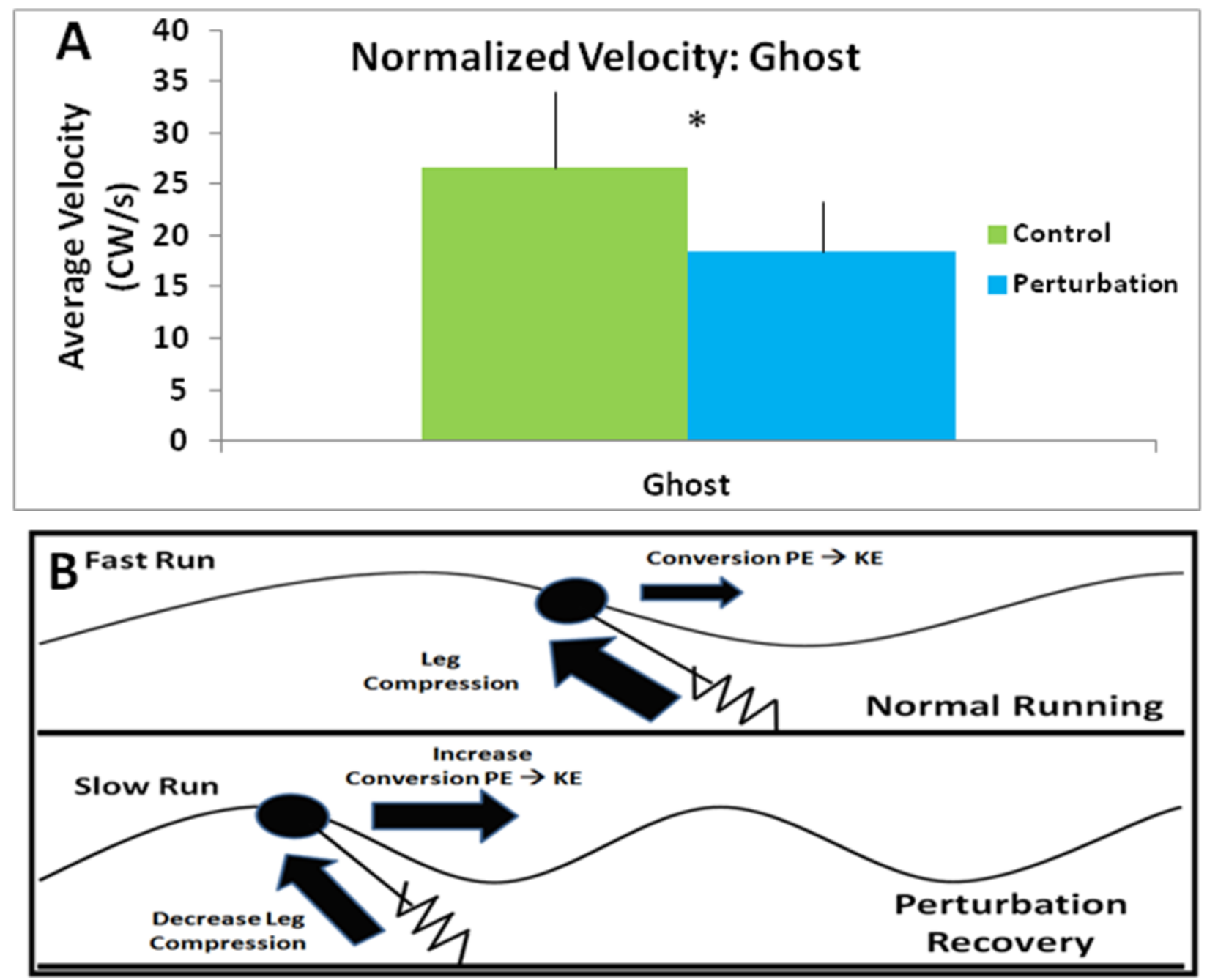

Figure 2- Average velocity and theoretical SLIP model: (A) Average normalized velocity of ghost crab running for control and perturbation running in carapace widths per second. (B) Theoretical spring loaded inverted pendulum model of a animal running at half the speed with the same vertical movement during each stride explaining why energy recovery can increase as velocity decreases. 
Figure 3: Halloween mechanical energy recovery
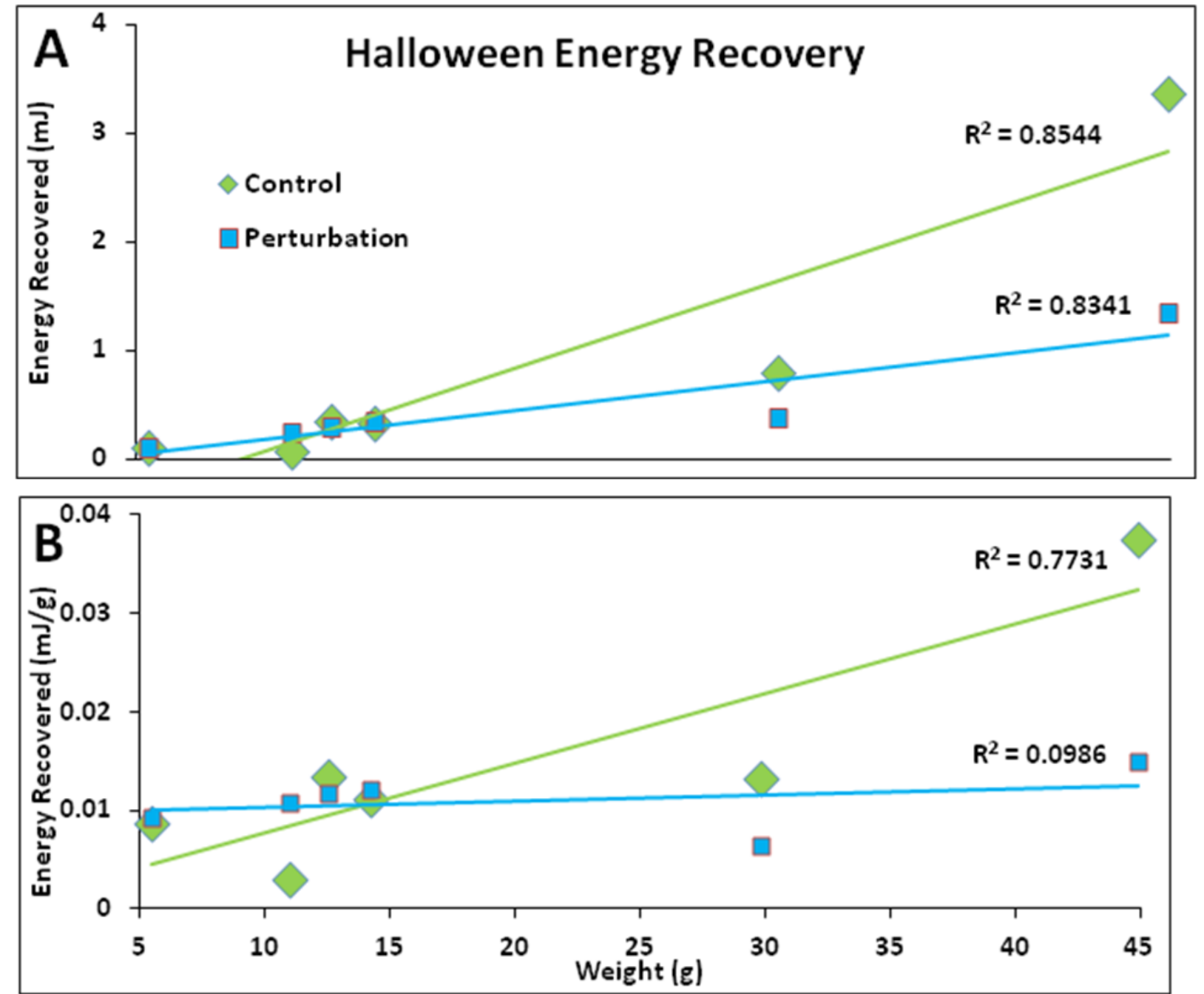

Figure 3- Halloween crab mechanical energy recovered and normalized mechanical energy recovered: Regression analysis of mechanical energy recovered (A) and normalized to weight (B) across weight ranges of animals studied. $\mathrm{Y}$-axis displays energy in $\mathrm{mJ}$ and $\mathrm{X}$-axis displays halloween crab weight in grams, $\mathrm{R}^{2}$ values are displayed for each trial type. 
Figure 4: Halloween crab lead $1^{\text {st }}$ CB joint perturbation response
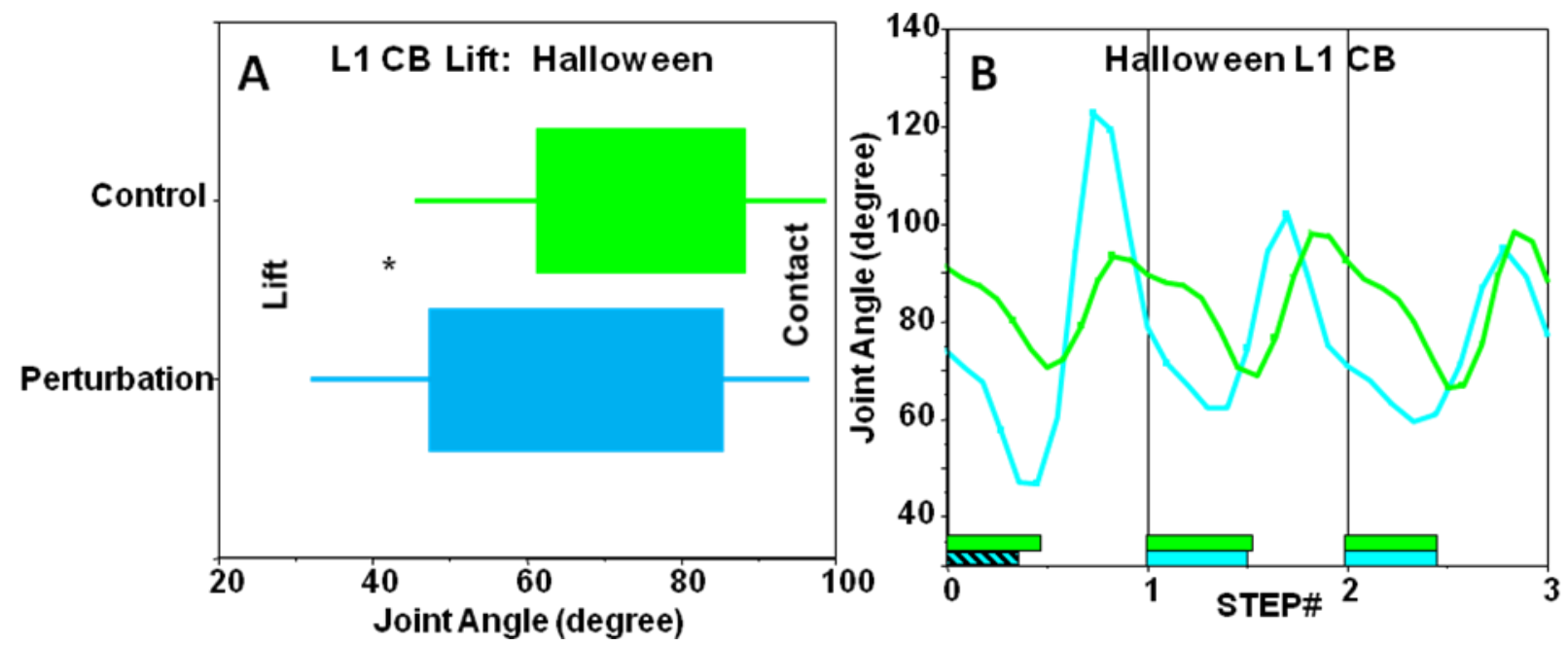

Figure 4-Halloween crab lead $1^{\text {st }} C B$ joint perturbation response- $(A)$ Average lift off and contact angle of the halloween crab $\mathrm{L} 1 \mathrm{CB}$ joint during control and perturbation recovery runs. Large bars indicate the range of angles between contact and liftoff angles and small bars represent standard deviation for each. (B) Individual trial for both control (green) and perturbation recovery (blue). The bars at the bottom display the proportion of the step that was spent in contact with substrate, striped blue bar indicates the step that the slip occurred. 
Figure 5: Halloween crab Trailing $3^{\text {rd }}$ TC joint perturbation response
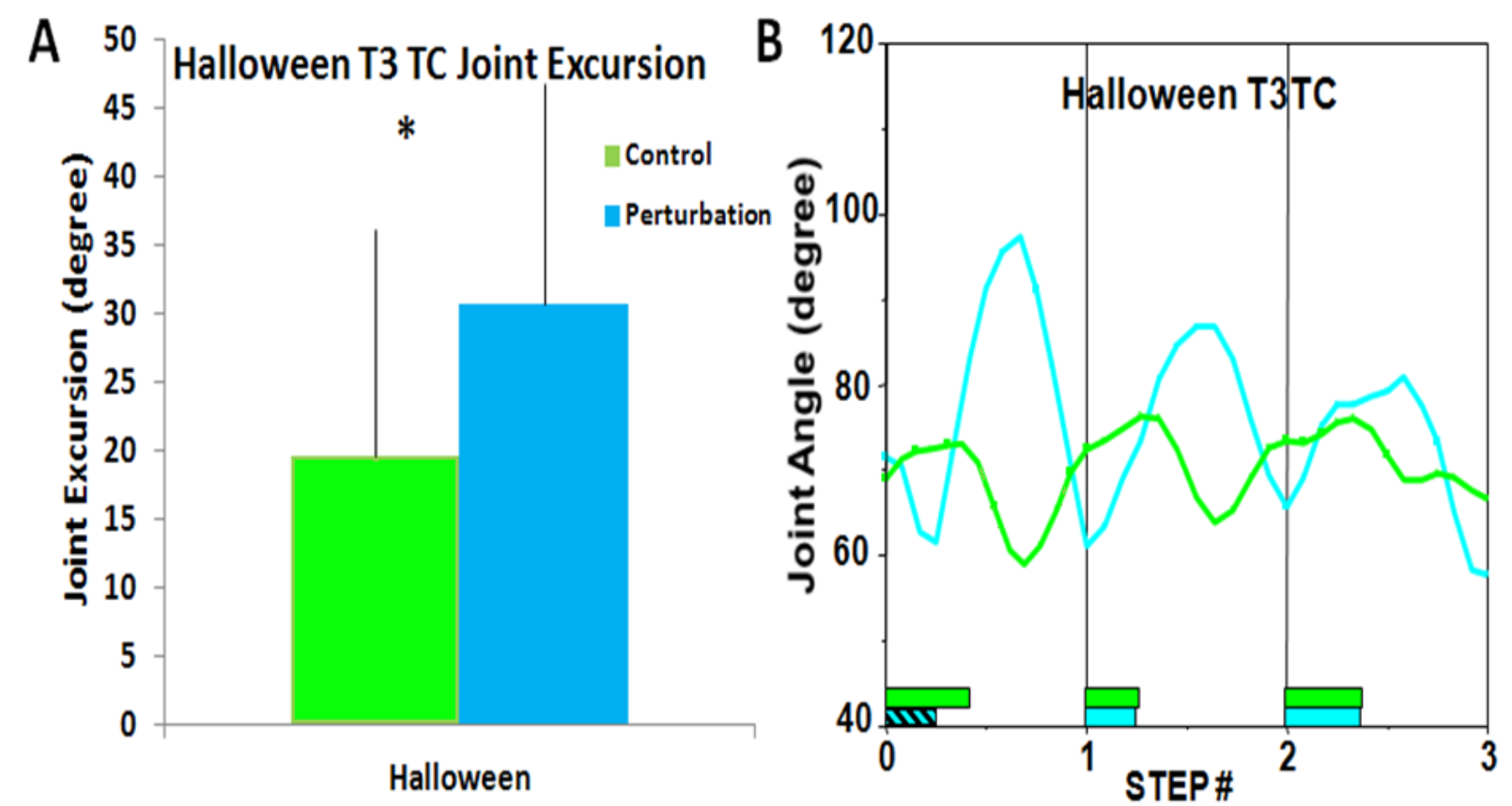

Figure 5- Halloween crab trailing $3^{\text {rd }}$ TC joint excursions: $(A)$ average and standard deviation of the excursions in the T3 TC joint during control and perturbation recovery running. (B) Individual joint tracings of the T3 TC joint following a slip, control (green) and perturbation recovery (blue). The bars at the bottom display the proportion of the step that was spent in contact with substrate, striped blue bar indicates the step that the slip occurred. 
Figure 6: Halloween Trailing $3^{\text {rd }}$ PD joint perturbation response
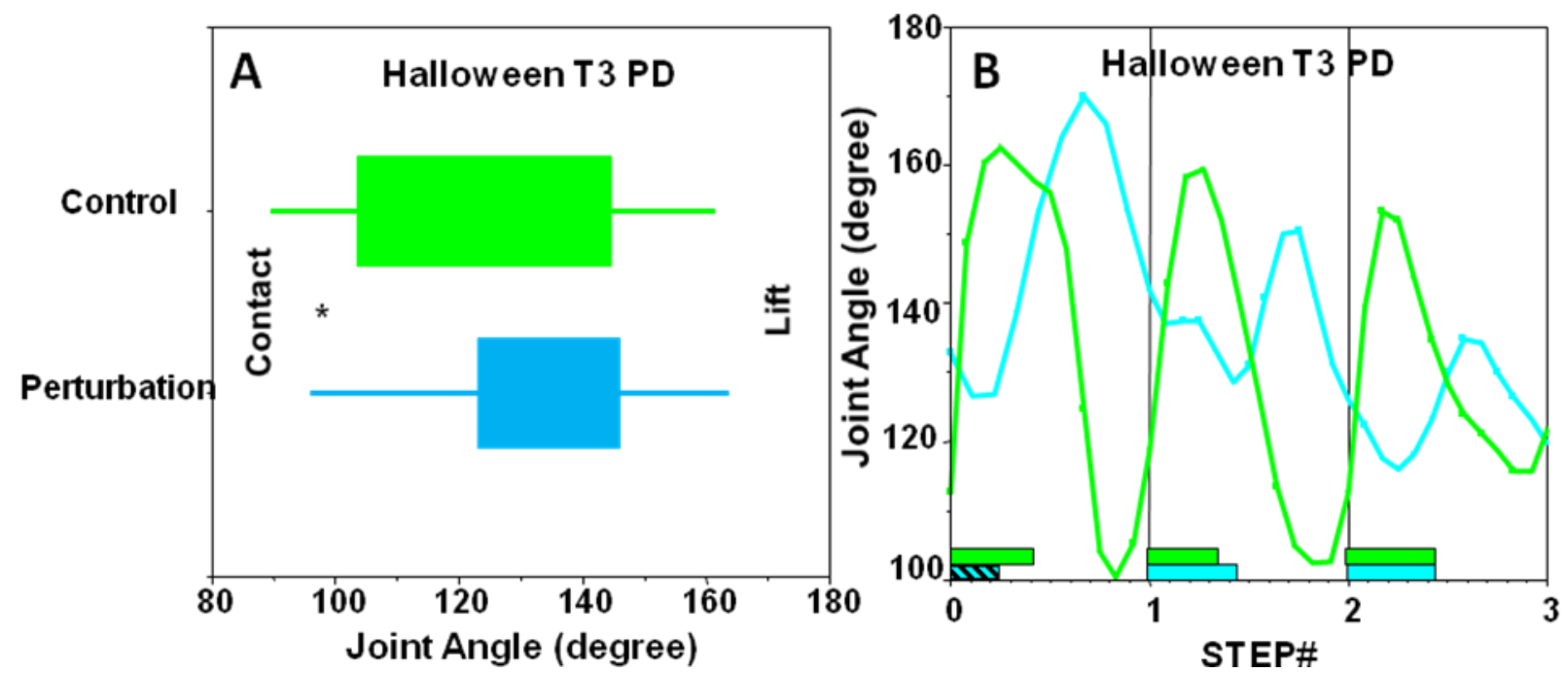

Figure 6-Halloween crab trailing $3^{\text {rd }}$ PD joint perturbation response- $(A)$ Average lift off and contact angle of the halloween crab T3 PD joint during control and perturbation recovery runs. Large bars indicate the range of angles between contact and liftoff angles, small bars represent standard deviation for each and the star represents a significant difference between the two. (B) Individual trial for both control (green) and perturbation recovery (blue). The bars at the bottom display the proportion of the step that was spent in contact with substrate, striped blue bar indicates the step that the slip occurred. 
Figure 7: Fiddler crab mechanical energy recovery
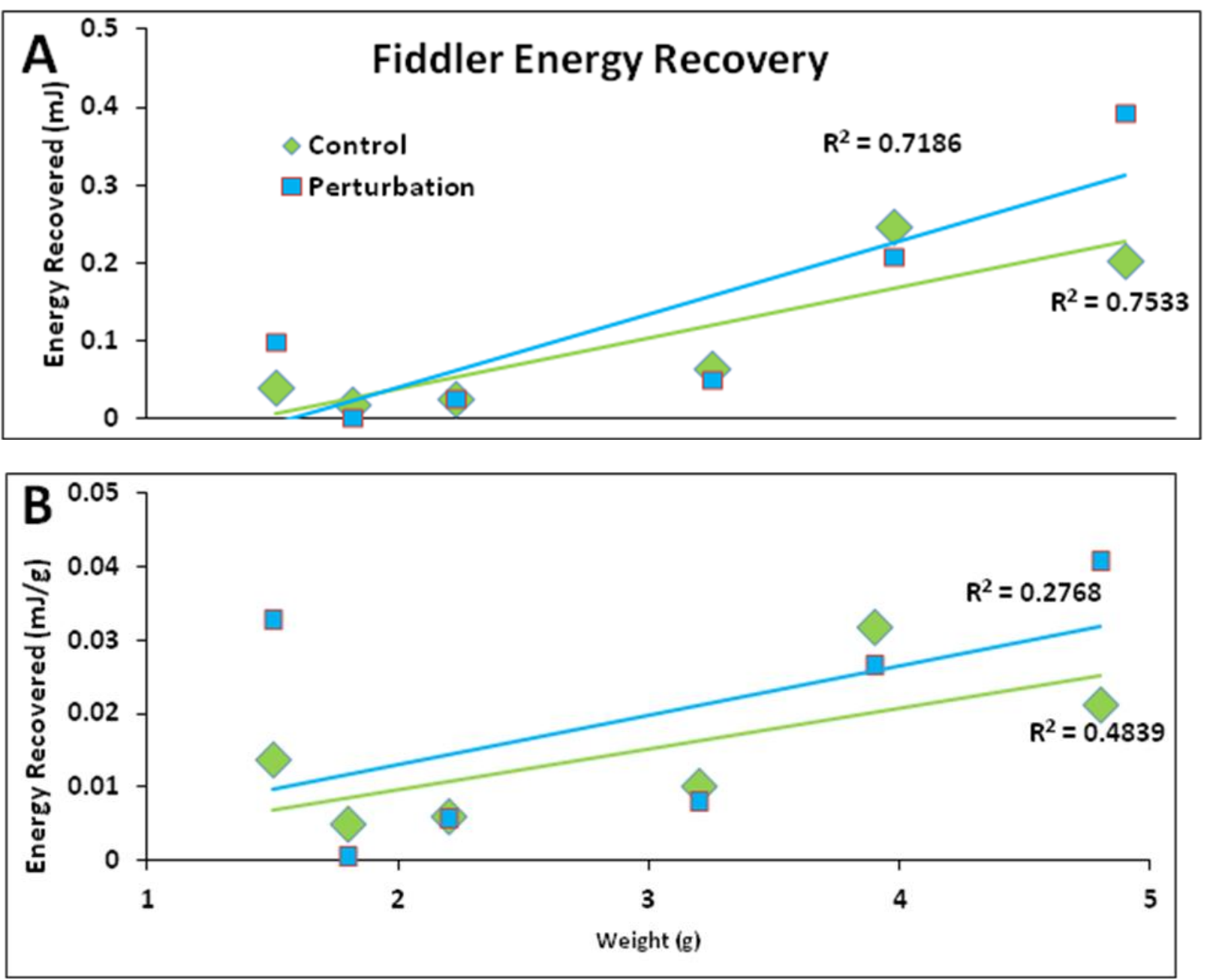

Figure 7- Fiddler crab mechanical energy recovered and normalized mechanical energy recovered: Regression analysis of mechanical energy recovered (A) and normalized to weight (B) across weight ranges of animals studied. $\mathrm{Y}$-axis displays energy in $\mathrm{mJ}$ and $\mathrm{X}$-axis displays fiddler crab weight in grams, $R^{2}$ values are displayed for each trial type. 
Figure 8: Fiddler Leading $1^{\text {st }}$ TC Joint

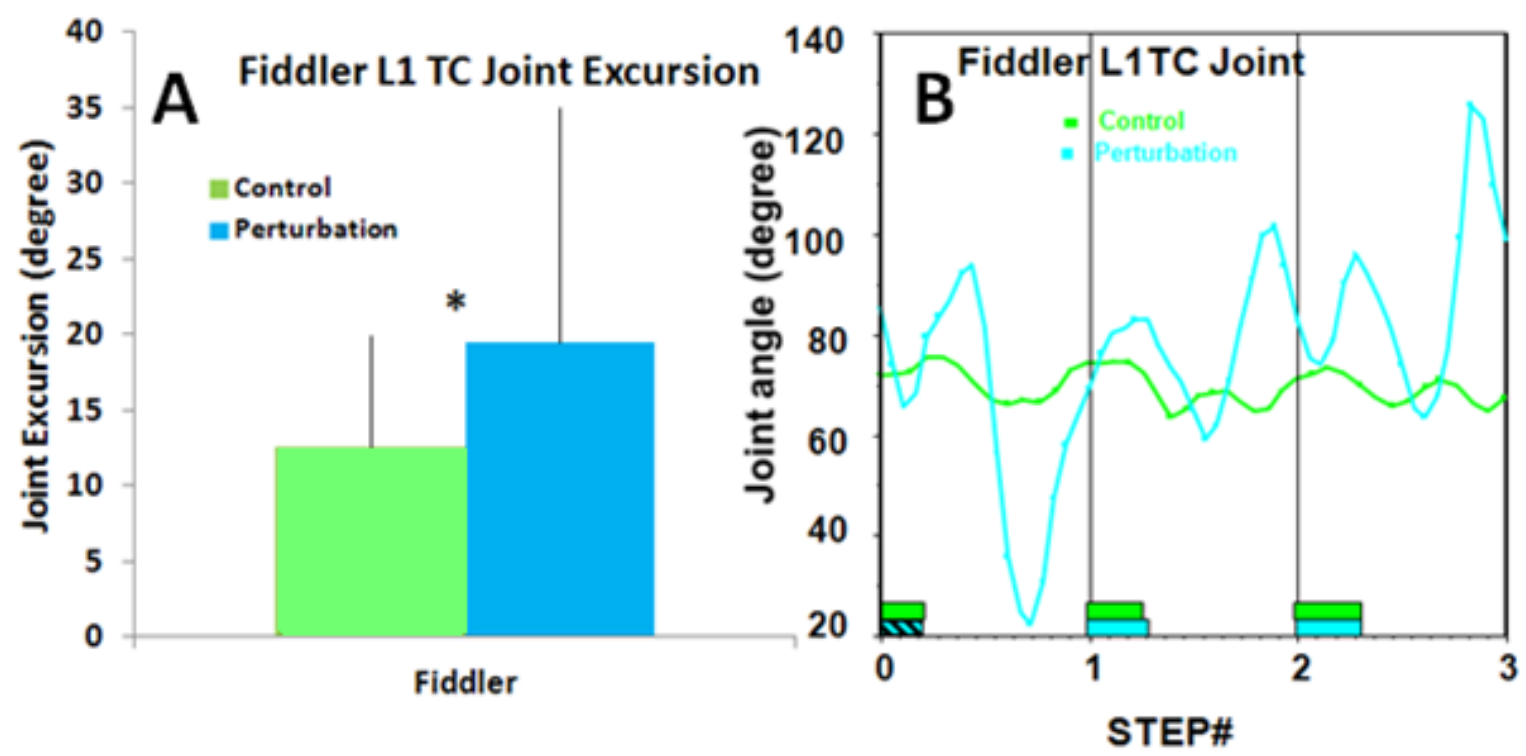

Figure 8- Fiddler crab Leading $\mathbf{1}^{\text {st }}$ TC joint excursions: (A) average and standard deviation of the excursions in the fiddler crab L1 TC joint during control and perturbation recovery running. (B) Individual joint tracings of the L1 TC joint following a slip, control (green) and perturbation recovery (blue). The bars at the bottom display the proportion of the step that was spent in contact with substrate, striped blue bar indicates the step that the slip occurred. 
Figure 9: Fiddler crab Leading $3^{\text {rd }}$ and $4^{\text {th }}$ MC Joint Contact

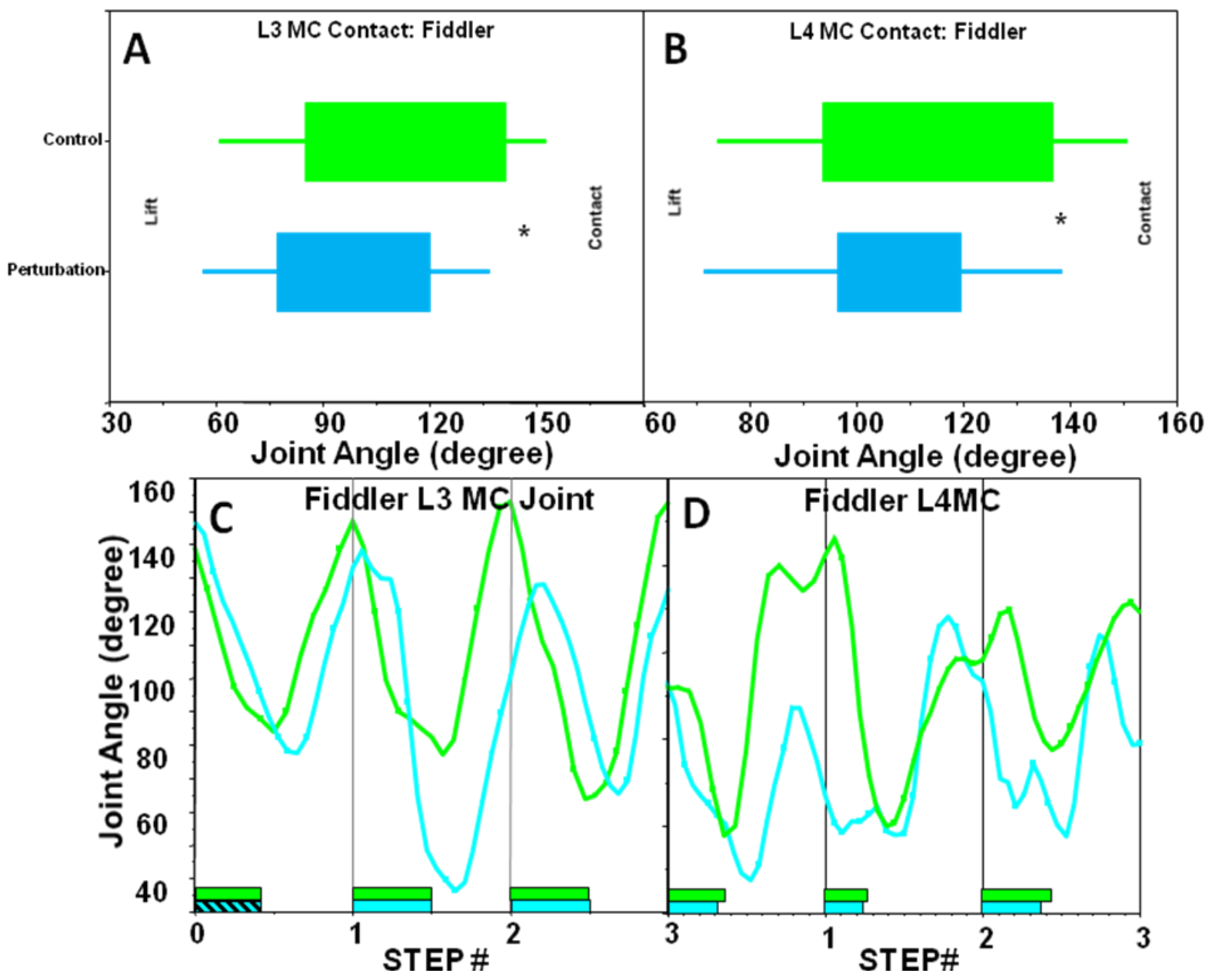

Figure 9-Fiddler crab leading $3^{\text {rd }}$ and $4^{\text {th }} \mathrm{MC}$ joint perturbation response- (A) Average lift off and contact angle of the fiddler crab L3 MC joint during control and perturbation recovery runs. (B) Average liftoff and contact angle of the fiddler crab L4 MC joint during control and perturbation recovery runs. Large bars indicate the range of angles between contact and liftoff angles, small bars represent standard deviation for each and the star represents a significant difference between the two trial types. (C) Individual tracing of the L3 MC joint for both control (green) and perturbation recovery (blue). (D) Individual tracing of the L4 MC joint for both control (green) and perturbation recovery (blue). The bars at the bottom display the proportion of the step that was spent in contact with substrate, striped blue bar indicates the step that the slip occurred. The L4 MC joint tracing is of the three steps following a lead $3^{\text {rd }}$ leg slip. 


\section{Chapter 4: Conclusion}




\section{Conclusion}

Movement through natural environments imposes evolutionary pressures that dictate animal design and physiology. These evolutionary pressures develop mechanical, neural and behavior traits that allow for efficient, rapid and adjustable locomotion. These traits produce unique locomotor capabilities that enable animals to survive and reproduce in their respective environments. Comparing how these unique locomotor designs function during normal and perturbed movement can elucidate important factors associated with locomotor capability and stability (Dickinson et al, 2001).

Brachyuran crabs provide an ideal model for determining the structural and behavior subtleties that produce locomotor differences across species. Most locomotor models provide examples of exaggerated capabilities such as running, jumping, endurance or efficiency. Studies of these model organisms focus on one aspect of a species movement that is an exemplar of a single trait produced through evolutionary pressures. Brachyuran crabs provide a group of model organisms that are specialized for reliable locomotion in a variety of environments. This variety of capabilities inlaid onto similar body structures permits the isolation of the structural and behavior variables responsible for differences in body dynamics during locomotion.

Evolutionary specializations enable movement in a variety of environments and provide insight into how evolution shapes the mechanical and behavioral properties of animal design. Determining how specialized design and control features produce movement can reveal concepts that are fundamental to understanding animal locomotion. These concepts can then be anchored in mathematical models to produce a quantitative representation of movement and provide inspiration for material design, robotics and prosthetics (Full and Koditschek, 1999). Although these concepts are inspirational for engineering, they must be fundamental enough to provide the necessary framework for locomotion without considering the entire animal. Manmade designs are currently unable to match the complexities of neural control and musculoskeletal mechanics, necessitating that artificial designs be built to mimic the fundamental movements without considering whole body dynamics (Ritzmann et al, 2004). 
Here we showed that body proportions, stepping patterns and joint activity can produce a range of gait dynamics. Ghost, halloween and fiddler crabs' ability show that despite very similar body layouts, slight changes in structure and joint behavior can drastically alter how normal running and perturbation recoveries are performed. These animal designs provide a framework to progress mathematical models and control theories for the study of animal movement and the creation of reliable robotics.

Lessons learned from comparative perturbation studies will be valuable to the future of locomotion research. This methodology places physical and behavioral traits in the context of adaptation and specialization, taking advantage of evolutionary pressures for insight into optimal locomotor strategies. Understanding how evolution shapes locomotor structures and behavior to adapt with the environment will provide a deeper knowledge of how locomotion is controlled across the animal kingdom. 


\section{References Cited}

Dickinson, M., Farley, C., Full, R., Koehl, M., Kram, R. and S. Lehman (2001) How animals move: an Integrative view. Science.288: 100-106

Full, R. and D. Koditschek. (1999) Templates and Anchors: Neuromechanical Hypotheses of Legged Locomotion on Land. Journal of Experimental Biology. 202: 3325-3332.

Nishikawa, K., Biewener, A. A., Aerts, P., Ahn, A. N., Chiel, J. H., Daley, M. A., Daniel, T. L., Full, R. J., Hale, M. E., Hedrick, T. L., Lappin A. K., Nichols, R., Quinn, R. D., Satterlie, R. A., and B. Szymik. (2007) Neuromechanics: an integrative approach for understanding motor control. Integrative and Comparative Biology. 47: 16-54

Ritzmann, R., Quinn, R. and M. Fischer (2004) Convergent evolution and locomotion through complex terrain by insects, vertebrates and robots. Arthropod Structure and Development. 33: 361-379 